\title{
Summary and Statistical Analysis of the First AIAA Sonic Boom Prediction Workshop
}

\author{
Michael A. Park,* \\ NASA Langley Research Center, Hampton, VA 23681 \\ John M. Morgenstern ${ }^{\dagger}$ \\ Lockheed Martin Aeronautics Company, Palmdale, CA 93599
}

\begin{abstract}
A summary is provided for the First AIAA Sonic Boom Workshop held 11 January 2014 in conjunction with AIAA SciTech 2014. Near-field pressure signatures extracted from computational fluid dynamics solutions are gathered from nineteen participants representing three countries for the two required cases, an axisymmetric body and simple delta wing body. Structured multiblock, unstructured mixed-element, unstructured tetrahedral, overset, and Cartesian cut-cell methods are used by the participants. Participants provided signatures computed on participant generated and solution adapted grids. Signatures are also provided for a series of uniformly refined workshop provided grids. These submissions are propagated to the ground and loudness measures are computed. This allows the grid convergence of a loudness measure and a validation metric (difference norm between computed and wind tunnel measured near-field signatures) to be studied for the first time. Statistical analysis is also presented for these measures. An optional configuration includes fuselage, wing, tail, flow-through nacelles, and blade sting. This full configuration exhibits more variation in eleven submissions than the sixty submissions provided for each required case. Recommendations are provided for potential improvements to the analysis methods and a possible subsequent workshop.
\end{abstract}

\section{Nomenclature}

$b \quad$ Wing span

dp/pinf Freestream pressure difference normalized by freestream

$h \quad$ Characteristic spacing of the grid

$H \quad$ Distance below the model

$K \quad$ Coverage factor

$L \quad$ Model reference length

$p \quad$ Pressure

$R \quad$ Radius from the model centerline

$V \quad$ Validation metric

$x_{0} \quad$ Forward validation metric integration extent

$x_{1} \quad$ Aft validation metric integration extent

$\beta \quad$ Tangent of freestream shock angle

$\tilde{\mu} \quad$ Estimate of sample median

$\tilde{\sigma} \quad$ Estimate of sample standard deviation

$\tau \quad X$, shifted and normalized by $(X-\beta R) / L$

$\phi \quad$ Off-track angle (zero is directly below model)

$\infty \quad$ Freestream conditions

${ }^{*}$ Research Scientist, Computational AeroSciences Branch, AIAA Senior Member.

${ }^{\dagger} \mathrm{N}+2$ Technical Manager, Advanced Development Programs, AIAA Associate Fellow. 


\section{Introduction}

The First American Institute of Aeronautics and Astronautics (AIAA) Sonic Boom Prediction Workshop (SBPW-1) was held on January 11, 2014 at National Harbor, Maryland. There were approximately 50 attendees from 7 nations. The objective of this workshop was to assess the state of the art for predicting near-field pressure signatures needed for accurate and reliable sonic boom prediction. These near-field pressure signatures, extracted from Computational Fluid Dynamics (CFD) solutions, were gathered from international participants. The participants utilized a series of uniformly refined workshop provided grids and grids created with each participant's best practices for computing solutions on the provided geometries.

Statistical analysis of the SBPW-1 submissions is facilitated by reducing the submitted near-field signatures to a set of scalars for N-version testing. ${ }^{1}$ SBPW-1 focuses on near-field CFD, but predicting the acoustic signature on the ground and how it is perceived by humans is the goal of the sonic boom community. The submitted near-field signatures are propagated to the ground (Fig. 1) and noise measures are computed to reduce these signatures to relevant scalars. The comparison of CFD to wind tunnel measurements is also of interest to the sonic boom community, so a validation metric is computed to quantify the difference between near-field CFD and measurements. The workshop requirement of computing solutions on uniformly refined grids allows, for the first time, the grid convergence of these scalars to be examined. The statistical products and grid convergence studies begin the process of quantifying the uncertainty of near-field CFD employed by the international participants.

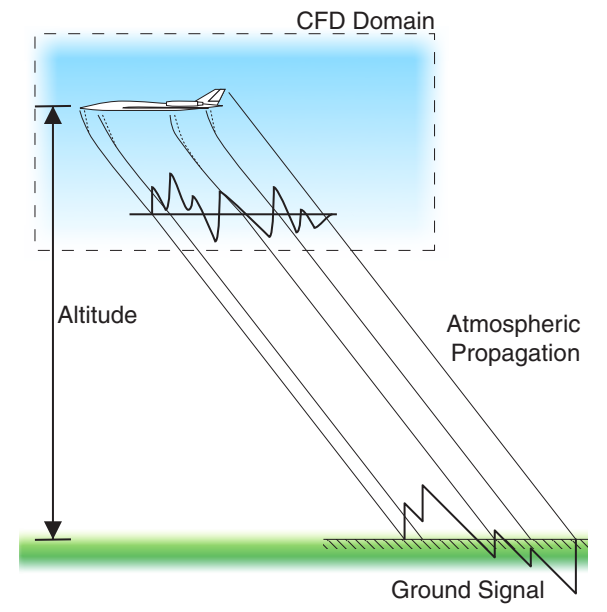

Figure 1. Sonic boom signal propagation. ${ }^{2}$

Waithe $^{3}$ introduced the workshop in special session at the $51^{\text {st }}$ AIAA Aerospace Sciences Meeting, a year before the workshop. The name of the workshop was changed and one of the required cases was replaced with the SEEB-ALR (computer program SEEB ${ }^{4}$ modified with Lockheed-Martin proprietary Aft Lift Relaxation) because as-built geometry was available and the model was larger in size. The larger model had longer shaped signature that enabled more accurate wind tunnel measurements. The DWB $\left(69^{\circ}\right.$ Delta Wing Body) ${ }^{5}$ was also chosen as a required case and the LM1021 (Lockheed-Martin 1021-01 configuration) ${ }^{6}$ was an optional case. See Fig. 2 for an illustration of the models. This special session included a summary of the 2008 NASA Sonic Boom Prediction Workshop, ${ }^{7}$ which also utilized the DWB model at a different flow condition, a number of axisymmetric bodies, and a configuration with wing, body, tails, and nacelles. The 2008 workshop had a much smaller scope than SBPW-1; it was limited to five NASA participants and the only analysis of results was comparing near-body signatures to wind tunnel measurements. SBPW-1 benefits from the lessons learned at the 2008 NASA Sonic Boom Prediction Workshop and previous AIAA workshops. $^{8-11}$

The data and analysis methods of this summary are intended to contribute toward the discussion of replacing the prohibition of overland supersonic flight ${ }^{13}$ with a certification standard. The existing ban on overland flight increases the cost and block time ${ }^{14}$ of supersonic fights between city pairs and is an important driver of the economic viability of a supersonic transport. ${ }^{15,16}$ 


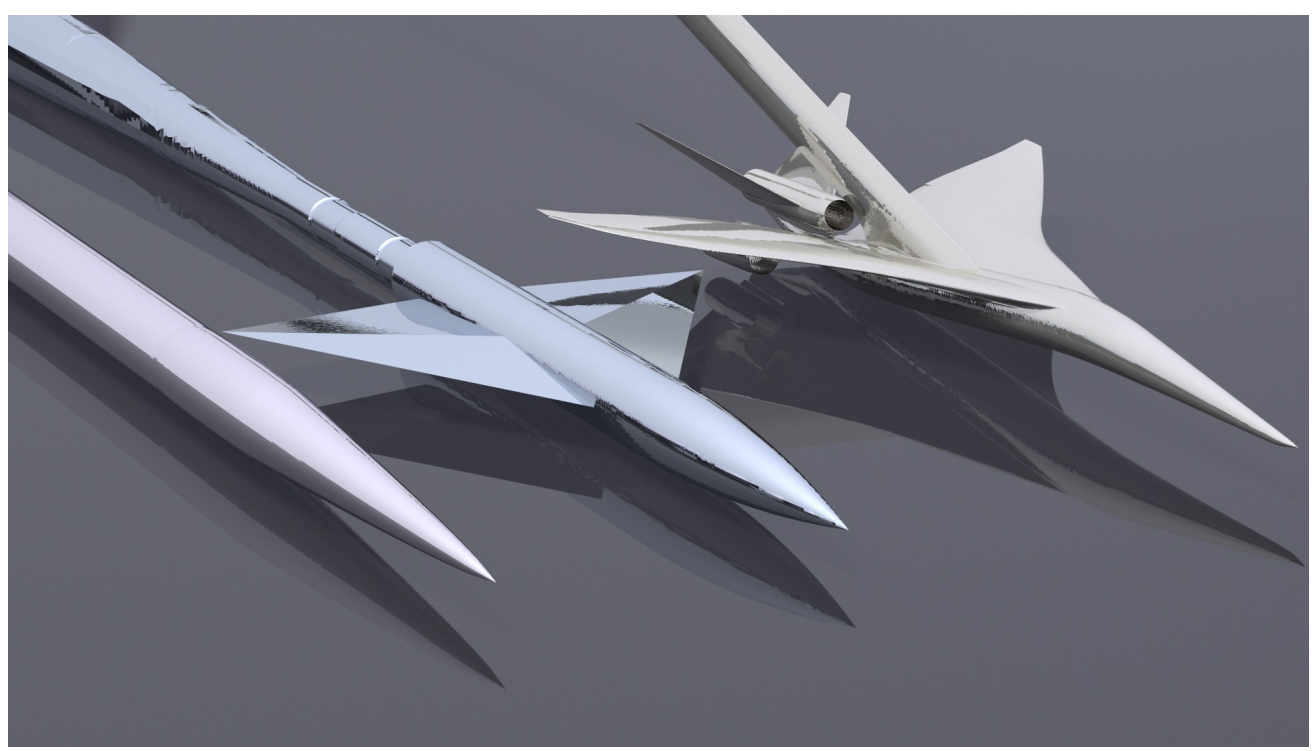

Figure 2. Rendering of SBPW-1 model discrete geometries shown left to right: SEEB-ALR, DWB, and LM1021. Not to relative scale. ${ }^{12}$

\section{Evaluation Methods}

The SBPW-1 participants submitted near-field pressure signatures: normalized delta pressure as a function of $x$. While these signatures could be compared directly to each other and wind tunnel measurements, it was helpful to reduce the properties of these signatures to scalars for grid refinement studies and application of statistical methods. This data reduction was performed by the authors, not by the participants, in an attempt to apply these methods as uniformly as possible to the submissions.

\section{II.A. Loudness}

Loudness and annoyance are inherently subjective measures of a sonic boom experience. Many noise descriptors have been evaluated as loudness predictors in human experiments, for example see Leatherwood et al. ${ }^{17}$ This summary utilizes two of them. The Mark VII perceived loudness level (PL) of Stevens ${ }^{18}$ is adopted as a measure to compare submissions because it is correlated with loudness and annoyance in multiple experiments. ${ }^{17}$ The C-weighted sound exposure level (CSEL) is also used because of its emphasis on a lower and wider range of frequencies. The method of Shepherd and Sullivan ${ }^{19}$ is used to calculate PL and CSEL from ground signatures. The propagation of the near-field submissions to the ground is described in the next section.

\section{II.B. Propagation}

Assumptions are made to scale the wind tunnel models and test conditions to a notional full-scale vehicle for propagation and loudness calculations. The wind tunnel test Mach number is used for the full-scale vehicle. All full-scale vehicles are assumed to be at 55,000 foot altitude in a no-wind US Standard Atmosphere. ${ }^{20}$ The 1.6 Mach SEEB-ALR wind tunnel model is 0.006 scale, ${ }^{21}$ the 1.7 Mach DWB wind tunnel model is 0.0065 scale ${ }^{3}$ and the 1.6 Mach LM1021 is 0.008 scale. ${ }^{21}$ Before propagation, the near-field pressure signature is scaled and the initial and final delta pressures are linearly ramped to zero, windowing the submission and wind tunnel measurements. The CFD submission computed with the largest grid for each configuration is shown with the wind tunnel (WT) measurement at the same location in Fig. 3. This windowing forces the initial and final pressures to freestream in an attempt to reduce the impact of wind tunnel mounting hardware on propagated signatures. Participants extracted pressure between different forward and aft locations. This windowing also eliminates these differences between submissions.

The sBOOM code of Rallabhandi ${ }^{22}$ is used to propagate near-field signatures to the ground using an augmented Burgers equation solver. This is the atmospheric propagation step in Fig. 1. Rallabhandi ${ }^{23}$ provides additional details of sBOOM including an adjoint formulation. ${ }^{23}$ Version 1.4 of sBOOM is used 


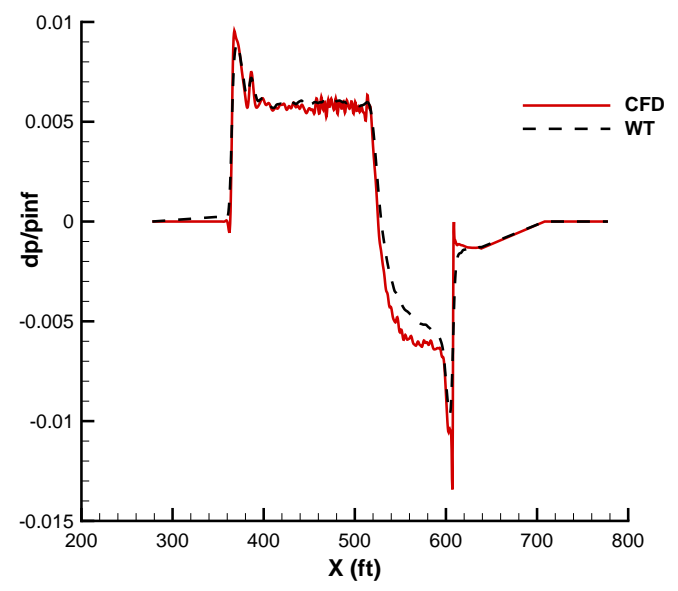

(a) SEEB-ALR.

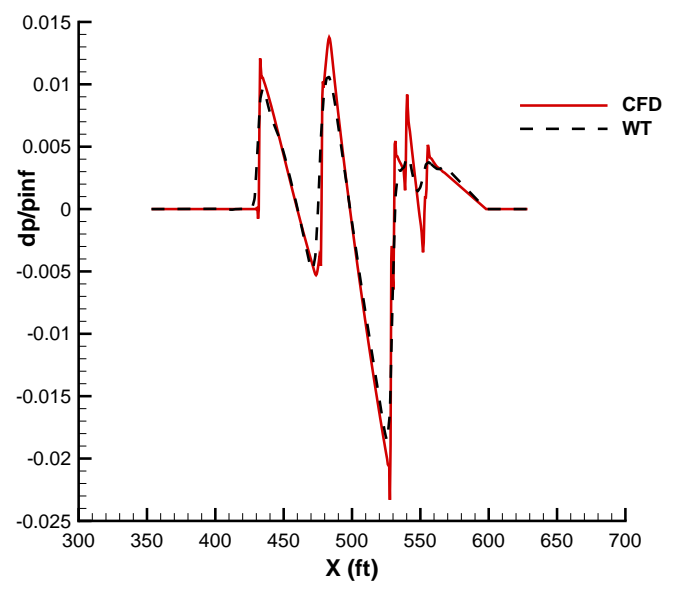

(b) DWB.

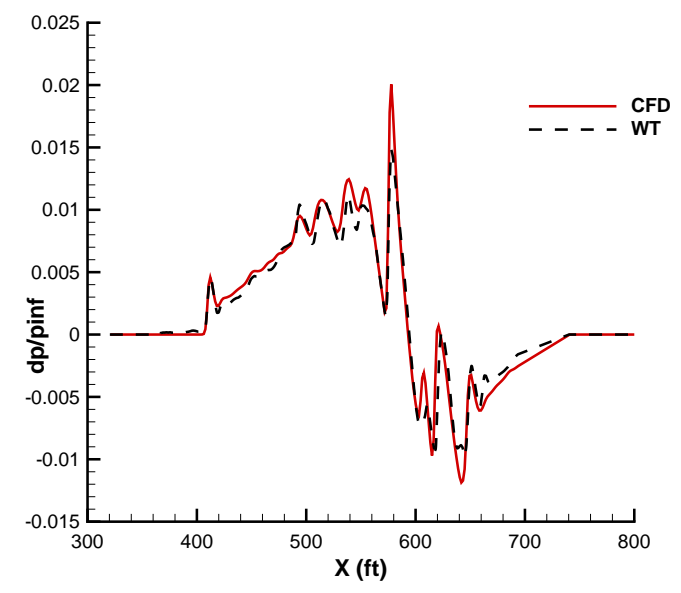

(c) LM1021.

Figure 3. Full-scale near-field signature with windowing. 
in this summary. It utilizes the atmospheric humidity model from ANSI S1.26, Annex C. ${ }^{24}$ The previous version 1.3 of sBOOM was used for the workshop overview and summary presentations. It had a bug in the atmospheric pressure calculation based on the hydrostatic equation and a different atmospheric humidity model. The changes for sBOOM version 1.4 resulted in a median PL increase that is approximately $1.2 \mathrm{~dB}$ louder for the SEEB-ALR and approximately $0.8 \mathrm{~dB}$ louder for the DWB than the statistics computed with version 1.3 .

Like the results of any finite-difference method, the ground signatures predicted by sBOOM has a sensitivity to the number of points used to discretize the signature. ${ }^{22}$ For example, Cleveland ${ }^{25}$ shows the dependency of the finite-difference molecular relaxation terms on the grid resolution. To verify the consistency of the sBOOM finite difference method, a series of uniformly refined grids are used. This refinement can be expressed as spatial refinement in the aircraft's reference frame (points per body length) or temporal refinement (sampling frequency) for a stationary (ground) observer. The resolution of the grid is expressed in points per body length and frequency in Fig. 4. Spatial resolution is important to the near-field CFD pressure extraction process and temporal resolution is important to interpreting results in the frequency space of the loudness calculation. PL as a function of sBOOM grid refinement is shown for wind tunnel measurements (WT) and the participant signature computed on the largest grid (CFD). The PL based on the LM1021 wind tunnel configuration is louder than predictions of a full-scale free-flight model due to a number of factors; see section V.C for details.

The sBOOM grid has 36,000 points for the generation of participant statistics, which corresponds to 18,000 points per body length (90K Hz sampling frequency) for the SEEB-ALR, 12,000 points per body length $(161 \mathrm{~K} \mathrm{~Hz}$ sampling frequency) for DWB, and 18,000 points per body length $(100 \mathrm{~K} \mathrm{~Hz}$ sampling frequency) for the LM1021. At this resolution, the computed PL is within a tenth of a $\mathrm{dB}$ of the asymptotic PL value. The LM1021 shows slightly different convergence behavior for CFD and WT below 18,000 points per body length (100K Hz sampling frequency). There is a nearly constant difference between the CFD and WT signatures for a range of resolutions for the SEEB-ALR and DWB, which indicates the statistics might be insensitive to resolution within this frequency range.

The default action of sBOOM is to detect the initial shock and interpolate the near field signature aft of this first shock to the available points. This was disabled to ensure that all signatures have equal resolution, because undershoots or smeared front shocks would result in a different effective resolution (points per body length).

The frequency spectra of the CFD ground signatures is shown in Fig. 5 when propagated with two different resolutions. This resolution is 18,000 points per body length $(31 \mathrm{KHz})$ and 6,000 points per body length $(93 \mathrm{KHz})$ for the SEEB-ALR and 12,000 points per body length $(161 \mathrm{KHz})$ and 4,000 points per body length $(53 \mathrm{KHz})$ for the DWB. The finer sBOOM grid only affects the higher frequencies (above $1 \mathrm{KHz}$ ). The SEEB-ALR has more energy in the lower frequencies (below $10 \mathrm{~Hz}$ ) than the DWB. The DWB has more energy above $10 \mathrm{~Hz}$ than the SEEB-ALR. The differences in the spectra are due to the SEEB-ALR flattop signature and the multiple N-wave character of the DWB (shown in Fig. 3), which agree with the analytic predictions of Shepherd and Sullivan. ${ }^{19}$

\section{II.C. Validation Metric}

A validation metric is employed to quantify the difference between an experimental measurement and a computational prediction. This technique was also employed in the analysis of the AIAA Shock BoundaryLayer Interaction Workshop submissions. ${ }^{11}$ For a detailed discussion of the construction and use of validation metrics, see Oberkampf and Roy. ${ }^{26}$ The wind tunnel measurements in this summary are the mean of an ensemble of measurements. See section VI for details of this averaging process used to extract the nearfield signature from the noisy environment in supersonic wind tunnel test section. The mean wind tunnel measurements are shifted in $x$ so that the location of the SEEB-ALR and LM1021 nose shocks and the DWB main expansion of the measurement mean matches the median of the participants. This difference is evaluated as an integral,

$$
V=\frac{1}{x_{1}-x_{0}} \int_{x_{0}}^{x_{1}}\left|\left(\frac{\Delta p}{p_{\infty}}\right)_{\text {submission }}-\left(\frac{\Delta p}{p_{\infty}}\right)_{\text {measurement }}\right| d x .
$$

The $x_{0}$ and $x_{1}$ locations define the same window used for each configuration during scaling for atmospheric propagation. Numerically, this integral is evaluated with the trapezoidal rule after all of the data points from 


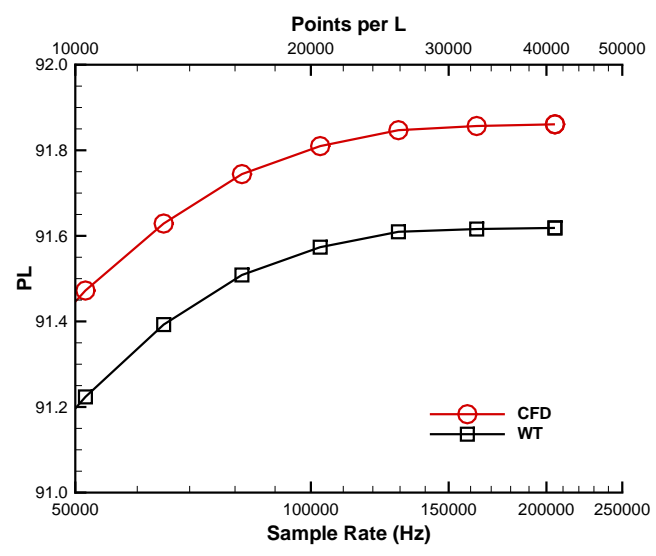

(a) SEEB-ALR.

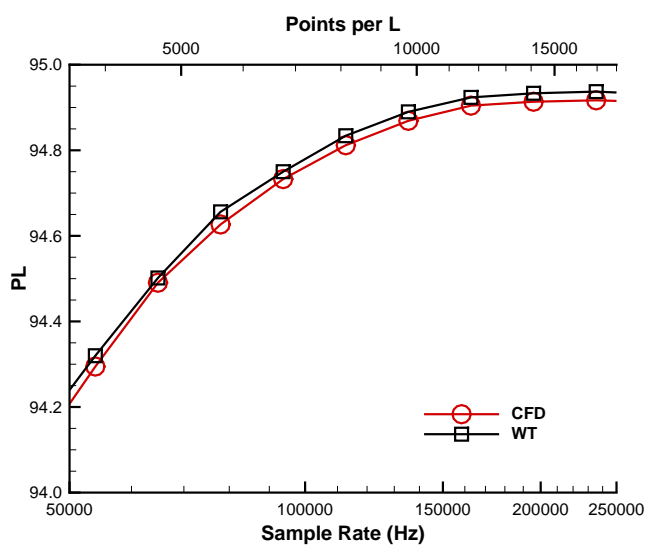

(b) DWB.

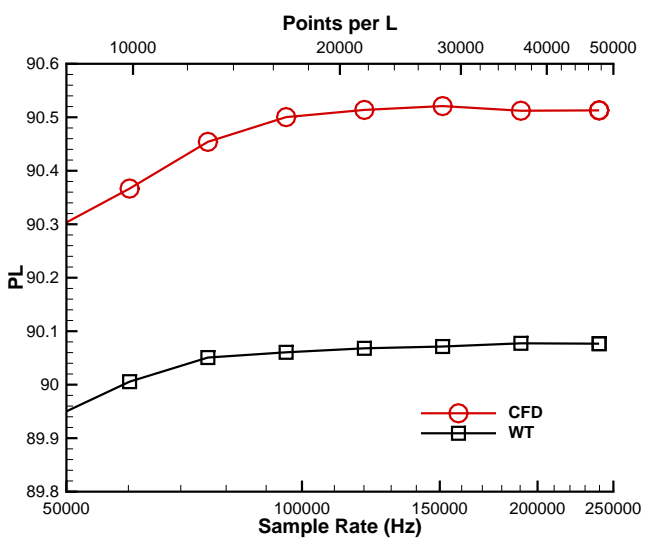

(c) LM1021.

Figure 4. Perceived Level as a function of sBOOM grid resolution.

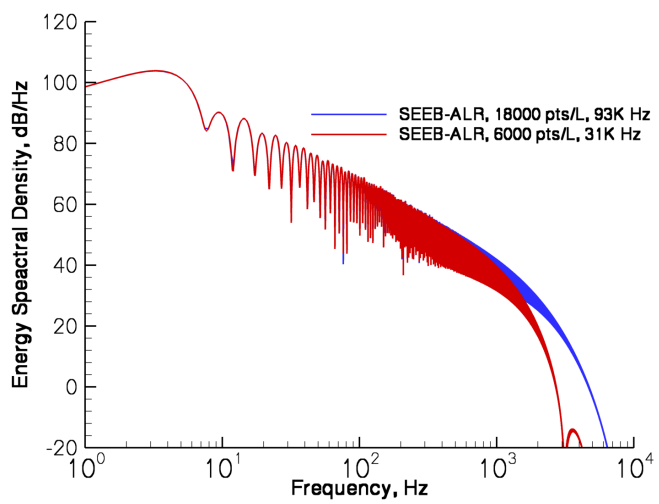

(a) SEEB-ALR

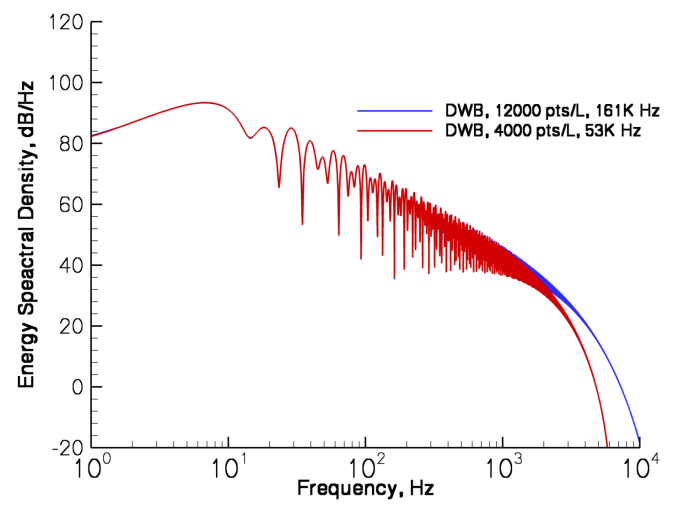

(b) DWB.

Figure 5. Ground signature frequency spectra at two sBOOM grid resolutions. 
the submission and mean wind tunnel measurement are linearly interpolated to the common $x$ locations of the submission and mean wind tunnel data.

\section{Statistical Approach}

The intention of this applied statistical approach is to measure the repeatability of an experiment that is run multiple times, where a workshop submission is treated as an experiment. In this context, no submission is treated as the "correct" prediction because all submissions contains known and unknown sources of uncertainty. The approach is based on the principles of the AIAA Drag Prediction Workshop (DPW) statistical summary. ${ }^{1,9}$ Two samples of the submissions are examined: a sample where the assumption of independence is violated by including uniformly refined grids from the same code and participant to increase the sample size, and a sample where the coarser uniformly refined grids are excluded. For each population, the scatter limits are established as

$$
\hat{\mu} \pm K \hat{\sigma} .
$$

The median is used to estimate the sample mean $\hat{\mu}$ because it is robust to outliers. The sample standard deviation $\hat{\sigma}$ is estimated with the corrected sample standard deviation. A coverage factor of $K=\sqrt{3}$ is calculated by assuming a uniform distribution of results because the actual distribution of the submissions is unknown. ${ }^{9}$ The first two DPW statistical summaries assumed $99 \%$ coverage of a normal distribution, which resulted in a larger $K{ }^{1}$ A submission is significant and considered outlier if it is outside the limits established by Eq. (2). A larger choice of $K$ would result in fewer submissions being outliers and a smaller choice of $K$ would result in more submissions being outliers.

\section{Participants}

The groups contributing to the workshop are listed in Table 1. The group letter identifier is included in the submission identifier throughout this summary. Some groups contain a single contributor, but most contain two or more collaborators. The groups have members from the United States of America, France, and Japan. Government agencies, industry, and academia are represented. Each group submitted results and prepared a presentation for the workshop. All group presentations are available on an FTP server, ftp:// lbpw-ftp.larc.nasa.gov/outgoing/lbpw1/presentations/, with the workshop introduction, overview, and summary presentations.

Table 1. Participant groups.

\begin{tabular}{cll} 
Group & Organizations & Contributors \\
\hline A & NASA Langley & M. Park \\
B & NASA Langley & S. Rallabhandi \\
C* & NASA Langley, NASA Ames & A. Elmiligui, S. Cliff \\
D & INRIA & A. Loseille, F. Alauzet \\
E & Dassault & F. Dagrau \\
F & ONERA & I. Salah El Din, A. Minelli, R. Grenon, G. Carrier \\
G & University of Miami & J. Gan, G. Zha \\
H & Stanford University, ONERA, INRIA & F. Palacios, T. Lukaczyk, J. Alonso, G. Carrier, A. Loseille \\
I & Lockheed Martin & J. Morgenstern \\
J & JAXA & H. Ishikawa, Y. Makino \\
K & Tokyo University of Science, JAXA & Z. Lei, Y. Makino \\
L & JAXA & M. Kanamori, A. Hashimoto, T. Aoyama \\
M & NASA Ames & M. Aftosmis, M. Nemec \\
N & Boeing & T. Magee, S. Fugal, D. Lazzara, M. Sexton, E. Unger \\
O & Gulfstream & D. Howe \\
P & NASA Langley & W. Li \\
Q & Lockheed Martin & M. Buonanno, F. Marconi \\
R & NASA Ames & J. Housman, E. Sozer, C. Kiris \\
S & Arizona State University & J. Jensen \\
\hline
\end{tabular}

* Group S submitted results after the workshop, but was included in the Group C workshop presentation. 


\section{Models and Required Grids}

The models for SBPW-1 were chosen to provide a range of geometric complexities, see Fig. 2. Grids were provided for both the required and optional cases in order to gain the largest degree of participation. Two cases were required: the SEEB-ALR and DWB. Participants were required to run at least one of the provided grids for these cases. The provided unstructured mixed-element and tetrahedral-only grids for the SEEB-ALR and DWB were described by Park et al. ${ }^{27}$ A series of uniformly refined grids were provided to quantify the spatial convergence of the near-field CFD schemes. The wide range of grid resolutions were practical for both node-centered and cell-centered schemes. Care was taken to ensure that the relative grid spacing distribution remained as similar as possible for the unstructured family of grids. The number of nodes and elements approximately doubled and a characteristic grid spacing decreased by the cube root of one half between successive grids in the series. ${ }^{27}$

Structured grids were also provided with instructions to recursively remove every other point in each computational direction to produce three coarser grids. Participants were encouraged to provide their own grids generated with best practices or solution adaptive methods. A single tetrahedral LM1021 grid was provided for the optional case as described by Morgenstern, Buonanno, and Marconi. ${ }^{6}$

\section{V.A. SEEB-ALR}

The SEEB-ALR model is described by Morgenstern et al. ${ }^{21}$ It is designed as an axisymmetric body, but slight asymmetry and other imperfections were introduced during wind tunnel model construction. These small imperfections in the geometry can be seen as high frequency, small amplitude pressure fluctuations in the flattop portion of the near-field signature, see Fig. 3(a). Resolving these pressure fluctuations is a deceptively difficult task and makes this an interesting configuration for the workshop. The SEEB-ALR solid model is constructed in $\mathrm{NX}^{\circledR}$ from a STEP file of the scanned, as-built wind tunnel model. The geometry differences between the as-designed and as-built are summarized by Cliff et al..$^{28}$ and Aftosmis and Nemec. ${ }^{12}$ The model has a reference length of $L=17.68 \mathrm{in}$.

The participants provided submissions at $H=21.2 \mathrm{in}$. and $H=42.0 \mathrm{in}$. below the centerline of the model, see Table 2. The SEEB submissions are listed in Table 3. The Groups are listed in Table 1. The Method is the CFD solver employed and Physics denotes the linearized Euler, Euler, Laminar, or Reynolds Averaged Navier-Stokes (RANS) with the specified turbulence model. Euler results were requested by the committee and the committee provided grids had a normal spacing intended for Euler calculation, so that is the most common submission. Type is a description of the construction of the grid. The Grid identifier is the grid generation (or adaptation) method: Output is output-based (goal-based) adaptation, PW is Pointwise ${ }^{\mathrm{TM}}$ commercial grid generation tool, $\mathrm{S} 1$ is the finest provided multiblock structured grids, and S2, $\mathrm{S} 3$, and $\mathrm{S} 4$ are recursively refined. The grids starting with $\mathrm{M}$ are committee-provided ${ }^{27}$ mixed-element grids and the grids starting with $\mathrm{T}$ are these mixed-element grids converted to all tetrahedra. The three numbers after the letter indicate the characteristic grid spacing $h$, where a smaller $h$ has more control volumes. The final column is the number of control volumes, which may be the number of nodes or cells depending on the CFD scheme. The submissions are provided for a large range for grid sizes. The smallest has 14 thousand control volumes and the largest has 73 million.

Table 2.
\begin{tabular}{llll} 
H & H $/ \mathrm{L}$ & $\phi$ & Available Wind Tunnel Measurements \\
\hline 21.2 in. & 1.2 & $0^{\circ}$ & Yes \\
42.0 in. & 2.4 & $0^{\circ}$ & Yes (poor quality) \\
\hline
\end{tabular}

\section{V.B. DWB}

The DWB (Fig. 6) was originally described as Model 4 in Hunton, Hicks, and Mendoza. ${ }^{29}$ It was selected for the workshop because it has a simple axisymmetric fuselage and diamond airfoil wing with available wind tunnel data both on and off the centerline. It provides a slight variation in on- and off-track near-field signature because the wing has different impact on the signature at different $\phi$ angles. This model was also of interest because many researchers have used it as a validation case, and the 2008 NASA Sonic Boom Prediction Workshop examined this model at angle of attack five years before SBPW-1. ${ }^{7}$ 
Table 3. SEEB-ALR submissions.

\begin{tabular}{|c|c|c|c|c|c|c|}
\hline Submission & Group & Method & Physics & Type & Grid & Cont. Vol. \\
\hline 1 & $A$ & CFL3D & Euler & Struct & S1 & $7,323,648$ \\
\hline 2 & A & CFL3D & Euler & Struct & $\mathrm{S} 2$ & 915,456 \\
\hline 3 & $A$ & CFL3D & Euler & Struct & S3 & 114,432 \\
\hline 4 & $A$ & CFL3D & Euler & Struct & S4 & 14,304 \\
\hline 5 & A & FUN3D & Euler & Mixed & M080 & $10,159,421$ \\
\hline 6 & A & FUN3D & Euler & Mixed & M100 & $5,252,466$ \\
\hline 7 & $A$ & FUN3D & Euler & Mixed & M125 & $2,537,977$ \\
\hline 8 & A & FUN3D & Euler & Mixed & M156 & $1,165,851$ \\
\hline 9 & A & FUN3D & Euler & Mixed & M200 & 674,734 \\
\hline 10 & $A$ & FUN3D & Euler & Tet & T080 & $10,159,421$ \\
\hline 11 & $A$ & FUN3D & Euler & Tet & T100 & $5,252,466$ \\
\hline 12 & A & FUN3D & Euler & Tet & T125 & $2,537,977$ \\
\hline 13 & A & FUN3D & Euler & Tet & T156 & $1,165,851$ \\
\hline 14 & $A$ & FUN3D & Euler & Tet & T200 & 674,734 \\
\hline 15 & B & FUN3D & Euler & Tet & VGRID & $21,519,091$ \\
\hline $16^{*}$ & C & USM3D & Euler & Tet & T080 & $59,565,426$ \\
\hline $17^{*}$ & C & USM3D & Euler & Tet & T100 & $30,647,394$ \\
\hline $18^{*}$ & $\mathrm{C}$ & USM3D & Euler & Tet & T125 & $14,695,383$ \\
\hline $19^{*}$ & C & USM3D & Euler & Tet & T156 & $6,662,284$ \\
\hline $20^{*}$ & $\mathrm{C}$ & USM3D & Euler & Tet & T200 & $3,840,249$ \\
\hline 21 & $C$ & USM3D & Euler & Tet & $\mathrm{PW} / \mathrm{MCAP}$ & $2,236,059$ \\
\hline 22 & $\mathrm{C}$ & USM3D & Laminar & Tet & $\mathrm{PW} / \mathrm{MCAP}$ & $6,196,118$ \\
\hline 23 & $\mathrm{C}$ & USM3D & SA & Tet & $\mathrm{PW} / \mathrm{MCAP}$ & $6,196,118$ \\
\hline $24^{*}$ & $\mathrm{D}$ & WOLF & Euler & Tet & Output & 208,280 \\
\hline $25^{*}$ & D & WOLF & Euler & Tet & Adapted & 875,511 \\
\hline $26^{*}$ & D & WOLF & Euler & Tet & T080 & $10,159,421$ \\
\hline $27^{*}$ & $\mathrm{D}$ & WOLF & Euler & Tet & $\mathrm{T} 100$ & $5,252,466$ \\
\hline $28^{*}$ & D & WOLF & Euler & Tet & T156 & $1,165,851$ \\
\hline $29^{*}$ & $\mathrm{D}$ & WOLF & Euler & Tet & T200 & $6,747,34$ \\
\hline 30 & $E$ & Eugenie & Euler & Tet & T080 & $10,159,421$ \\
\hline 31 & $E$ & Eugenie & Euler & Tet & T100 & $5,252,466$ \\
\hline 32 & $E$ & Eugenie & Euler & Tet & $\mathrm{T} 125$ & $2,537,977$ \\
\hline 33 & $E$ & Eugenie & Euler & Tet & T156 & $1,165,851$ \\
\hline 34 & $E$ & Eugenie & Euler & Tet & T200 & 674,734 \\
\hline 35 & $\mathrm{~F}$ & CEDRE & Euler & Tet & T100 & $30,647,394$ \\
\hline 36 & $\mathrm{~F}$ & CEDRE & Euler & Tet & T156 & $6,662,284$ \\
\hline 37 & $\mathrm{~F}$ & CEDRE & Euler & Tet & T200 & $3,840,249$ \\
\hline 38 & G & FASIP & Euler & Struct & $\mathrm{S} 1$ & $7,323,648$ \\
\hline 39 & $\mathrm{H}$ & SU2 & Euler & Mixed & M080 & $10,159,421$ \\
\hline 40 & $\mathrm{H}$ & SU2 & Euler & Mixed & M100 & $5,252,466$ \\
\hline 41 & $\mathrm{H}$ & SU2 & Euler & Mixed & M156 & $1,165,851$ \\
\hline 42 & $\mathrm{H}$ & SU2 & Euler & Mixed & M200 & 674,734 \\
\hline 43 & 1 & Linear & Linear & $\mathrm{N} / \mathrm{A}$ & $\mathrm{N} / \mathrm{A}$ & $\mathrm{N} / \mathrm{A}$ \\
\hline 44 & $J$ & TAS & Euler & Mixed & M080 & $27,865,176$ \\
\hline 45 & $J$ & TAS & Euler & Mixed & M100 & $14,325,194$ \\
\hline 46 & $J$ & TAS & Euler & Mixed & M200 & $1,793,549$ \\
\hline 47 & $J$ & UPACS & Euler & Struct & $\mathrm{S} 1$ & $7,323,648$ \\
\hline 48 & $\mathrm{~J}$ & UPACS & Euler & Overset & Fixed & $27,646,000$ \\
\hline 49 & $\mathrm{~K}$ & ADCS & Euler & Struct & $\mathrm{S} 1$ & $7,986,107$ \\
\hline 50 & $\mathrm{~L}$ & FaSTAR & Euler & Mixed & M100 & $10,159,421$ \\
\hline 51 & $\mathrm{~L}$ & FaSTAR & Euler & Mixed & M125 & $5,252,466$ \\
\hline 52 & $\mathrm{~L}$ & FaSTAR & Euler & Mixed & M156 & $1,165,851$ \\
\hline 53 & $\mathrm{~L}$ & FaSTAR & Euler & Mixed & M200 & 674,734 \\
\hline 54 & M & Cart3D & Euler & Cart & Output & $2,012,184$ \\
\hline 55 & $\mathrm{~N}$ & Cart3D & Euler & Cart & Fixed & $11,147,363$ \\
\hline 56 & $\mathrm{~N}$ & OVERFLOW & Euler & Overset & PW & $26,309,760$ \\
\hline 57 & $\mathrm{O}$ & CartOver & Euler & Hybrid & Fixed & $73,000,000$ \\
\hline 58 & $\mathrm{P}$ & Cart3D & Euler & Cart & Fixed & $18,096,942$ \\
\hline 59 & Q & CFD ++ & SA & Struct & Gridgen & $2,000,000$ \\
\hline 60 & $\mathrm{R}$ & LAVA & Euler & Overset & Fixed & $21,800,000$ \\
\hline 61 & $\mathrm{R}$ & LAVA & SA & Overset & Fixed & $21,800,000$ \\
\hline 62 & $\mathrm{R}$ & LAVA & Euler & Struct & Axi & 330,000 \\
\hline 63 & $\mathrm{R}$ & LAVA & SST & Struct & Axi & 330,000 \\
\hline $64^{*}$ & $\mathrm{~S}$ & OVERFLOW & SA & Overset & Fixed & $21,300,000$ \\
\hline
\end{tabular}

* Updated or submitted after the workshop.

9 of 43 


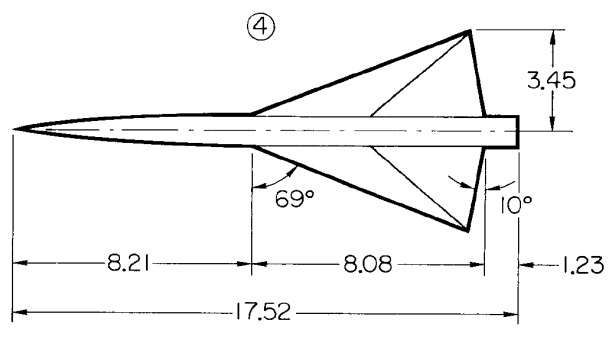

(a) DWB planform, dimensions in $\mathrm{cm}^{29}$

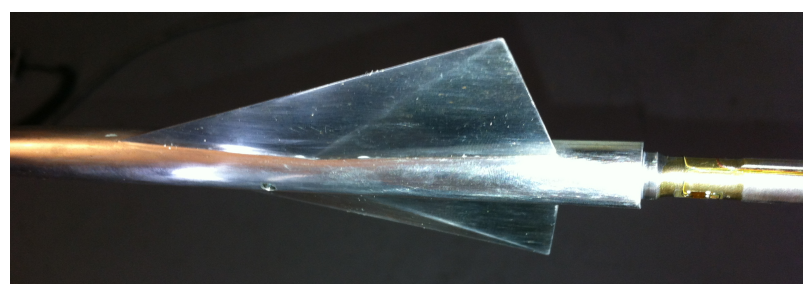

(b) DWB wind tunnel model and sting.

Figure 6. Delta Wing Body and wind tunnel mounting sting.

The DWB fuselage has an aft-facing step base region where it attaches to the wind tunnel sting, see Fig. 6(b). The solid model of the DWB is extracted from a STEP file exported from CATIA ${ }^{\circledR}$ V5, where it was created from the analytic description in the original report. ${ }^{5}$ The sting geometry was created from drawings used to machine the wind tunnel sting. A simplification was made by omitting the strain gauges, wires, and instrumentation slots visible in a photograph of the model, see Fig. 6(b). The sting was inserted 0.079184 meters ( 3.1175 inches) into the wing body and a Boolean addition produced the solid model. The model has a reference length of $L=0.1752 \mathrm{~m}$ and a half span of $b / 2=0.0345 \mathrm{~m}$, which produces a reference length to half span ratio of 5.1 .

The participants provided submissions containing ten extraction locations both on and off the centerline of the model, Table 4. The DWB submissions are listed in Table 5. See section V.A for a description of the abbreviations that are common for the SEEB-ALR and DWB tables.

Table 4. DWB pressure signature extraction locations.

\begin{tabular}{lllll}
$H$ & $H / L$ & $H /(b / 2)$ & $\phi$ & Available Wind Tunnel Measurements \\
\hline 0.5 in. & 0.07 & 0.34 & $0^{\circ}$ & No \\
21.2 in. & 3.07 & 13.4 & $0^{\circ}$ & No \\
24.8 in. & 3.60 & 16.7 & $0^{\circ}, 30^{\circ}, 60^{\circ}, 90^{\circ}$ & Yes \\
31.8 in. & 4.61 & 21.5 & $0^{\circ}, 30^{\circ}, 60^{\circ}, 90^{\circ}$ & Yes \\
\hline
\end{tabular}

\section{V.C. LM1021}

The NASA High Speed Project sponsored wind tunnel testing to validate very low sonic boom designs. ${ }^{21}$ The resulting measurements were found to match CFD predictions within $1 \mathrm{PL}(\mathrm{dB})$ when propagated to the ground. ${ }^{6}$ Based on this success, the LM1021 model (Fig. 7) was selected as a case for the workshop. It was a challenging candidate because analysis performed during model development indicated that the near-field signature changed when laminar or turbulent CFD was applied. This sensitivity to boundary layer state implied that near-body signatures extracted from Euler calculations could be different from viscous CFD calculations. Extensive wind tunnel model oil surface flow photos were captured for comparison with CFD analyses and to provide an understanding of boundary layer state and flow features. This full configuration model included wing, body, V-tail, and three nacelles that provide a signature that varies with off-track angle.

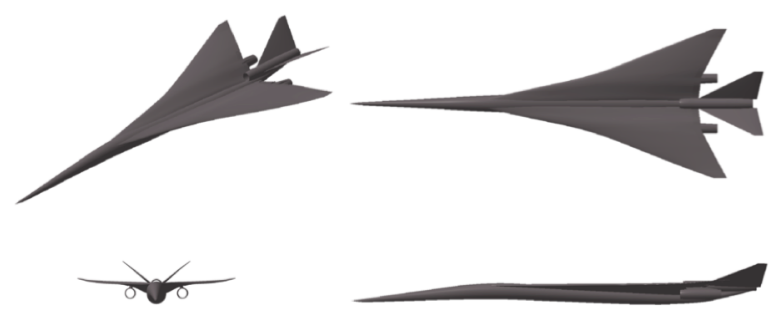

Figure 7. Perspective and three-view of the LM1021 free-flight model.

For non-axisymmetric bodies, the spanwise distribution of acoustic disturbance sources reach a near-field 
Table 5. DWB submissions.

\begin{tabular}{|c|c|c|c|c|c|c|}
\hline Submission & Group & Method & Physics & Type & Grid & Cont. Vol. \\
\hline 1 & A & CFL3D & Euler & Struct & S1 & $10,141,696$ \\
\hline 2 & A & CFL3D & Euler & Struct & $\mathrm{S} 2$ & $1,267,712$ \\
\hline 3 & $A$ & CFL3D & Euler & Struct & S3 & 158,464 \\
\hline 4 & $A$ & CFL3D & Euler & Struct & S4 & 19,808 \\
\hline 5 & $A$ & FUN3D & Euler & Mixed & M100 & $5,363,065$ \\
\hline 6 & $A$ & FUN3D & Euler & Mixed & M125 & $2,742,341$ \\
\hline 7 & A & FUN3D & Euler & Mixed & M156 & $1,411,515$ \\
\hline 8 & A & FUN3D & Euler & Mixed & M200 & 695,709 \\
\hline 9 & $A$ & FUN3D & Euler & Tet & T100 & $5,363,065$ \\
\hline 10 & A & FUN3D & Euler & Tet & T125 & $2,742,341$ \\
\hline 11 & A & FUN3D & Euler & Tet & T156 & $1,411,515$ \\
\hline 12 & A & FUN3D & Euler & Tet & T200 & 695,709 \\
\hline 13 & B & FUN3D & Euler & Tet & VGRID & $16,700,946$ \\
\hline $14^{*}$ & $\mathrm{C}$ & USM3D & Euler & Tet & T100 & $31,801,283$ \\
\hline 15 & $\mathrm{C}$ & USM3D & Euler & Tet & T125 & $16,191,339$ \\
\hline 16 & $\mathrm{C}$ & USM3D & Euler & Tet & T156 & $8,288,192$ \\
\hline $17^{*}$ & $\mathrm{C}$ & USM3D & Euler & Tet & T200 & $4,056,575$ \\
\hline 18 & $\mathrm{C}$ & USM3D & Euler & Tet & $\mathrm{PW} / \mathrm{MCAP}$ & $6,033,621$ \\
\hline 19 & $\mathrm{C}$ & USM3D & SA & Tet & $\mathrm{PW} / \mathrm{MCAP}$ & $7,864,672$ \\
\hline $20^{*}$ & $\mathrm{D}$ & WOLF & Euler & Tet & Adapted & 759,048 \\
\hline $21^{*}$ & $\mathrm{D}$ & WOLF & Euler & Tet & Adapted & 860,010 \\
\hline $22^{*}$ & $\mathrm{D}$ & WOLF & Euler & Tet & $\mathrm{T} 100$ & $5,363,065$ \\
\hline $23^{*}$ & $\mathrm{D}$ & WOLF & Euler & Tet & T125 & $2,742,341$ \\
\hline $24^{*}$ & $\mathrm{D}$ & WOLF & Euler & Tet & T156 & $1,411,515$ \\
\hline $25^{*}$ & $\mathrm{D}$ & WOLF & Euler & Tet & T200 & 695,709 \\
\hline 26 & $E$ & Eugenie & Euler & Tet & T100 & $5,363,065$ \\
\hline 27 & $E$ & Eugenie & Euler & Tet & T125 & $2,742,341$ \\
\hline 28 & $E$ & Eugenie & Euler & Tet & T156 & $1,411,515$ \\
\hline 29 & $E$ & Eugenie & Euler & Tet & T200 & 695,709 \\
\hline 30 & $\mathrm{~F}$ & CEDRE & Euler & Tet & T100 & $31,801,283$ \\
\hline 31 & $\mathrm{~F}$ & CEDRE & Euler & Tet & T200 & $4,056,575$ \\
\hline 32 & $\mathrm{~F}$ & elsA & Euler & Struct & $\mathrm{S} 1$ & $10,141,696$ \\
\hline 33 & G & FASIP & Euler & Struct & Gridgen & $9,848,768$ \\
\hline 34 & $\mathrm{H}$ & SU2 & Euler & Tet & Output & 229,000 \\
\hline 35 & $\mathrm{H}$ & SU2 & Euler & Tet & Adapted & $1,197,548$ \\
\hline 36 & $\mathrm{H}$ & SU2 & Euler & Mixed & M100 & $5,363,065$ \\
\hline 37 & $\mathrm{H}$ & SU2 & Euler & Mixed & M125 & $2,742,341$ \\
\hline 38 & $\mathrm{H}$ & SU2 & Euler & Mixed & M156 & $1,411,515$ \\
\hline 39 & $\mathrm{H}$ & SU2 & Euler & Mixed & M200 & 695,709 \\
\hline 40 & $J$ & UPACS & Euler & Struct & $\mathrm{S} 1$ & $10,141,696$ \\
\hline 41 & $J$ & UPACS & Euler & Overset & Fixed & $27,646,000$ \\
\hline 42 & $J$ & TAS & Euler & Mixed & M100 & $22,423,283$ \\
\hline 43 & $J$ & TAS & Euler & Mixed & M125 & $11,451,499$ \\
\hline 44 & $J$ & TAS & Euler & Mixed & M156 & $5,877,312$ \\
\hline 45 & $J$ & TAS & Euler & Mixed & M200 & $2,830,575$ \\
\hline 46 & $\mathrm{~K}$ & ADCS & Euler & Struct & Gridgen & $10,640,580$ \\
\hline 47 & $L$ & FaSTAR & Euler & Mixed & M100 & $5,363,065$ \\
\hline 48 & $L$ & FaSTAR & Euler & Mixed & M125 & $2,742,341$ \\
\hline 49 & 1 & FaSTAR & Euler & Mixed & M156 & $1,411,515$ \\
\hline 50 & L & FaSTAR & Euler & Mixed & M200 & 695,709 \\
\hline 51 & M & Cart3D & Euler & Cart & Output & $15,885,435$ \\
\hline 52 & $\mathrm{~N}$ & Cart3D & Euler & Cart & Adapted & $49,000,000$ \\
\hline 53 & $\mathrm{~N}$ & Cart3D & Euler & Cart & Fixed & $75,000,000$ \\
\hline 54 & $\mathrm{~N}$ & CFD ++ & Euler & Mixed & M100 & $22,423,283$ \\
\hline 55 & $\mathrm{O}$ & CartOver & Euler & Hybrid & Fixed & $108,000,000$ \\
\hline 56 & $\mathrm{P}$ & Cart3D & Euler & Cart & Fixed & $54,466,264$ \\
\hline 57 & Q & CFD ++ & Euler & Mixed & M100 & $22,423,283$ \\
\hline 58 & $\mathrm{R}$ & LAVA & SA & Overset & Fixed & $21,300,000$ \\
\hline 59 & $\mathrm{R}$ & LAVA & SA & Mixed & Fixed & $12,100,000$ \\
\hline $60^{*}$ & $\mathrm{~S}$ & OVERFLOW & SA & Overset & Fixed & $29,600,000$ \\
\hline
\end{tabular}

* Updated or submitted after the workshop. 
extraction location after traveling a longer distance than the centerline disturbances. This extra distance results in wave superposition at a more aft location that alters the signature shape. This signature change with extraction distance is known as near-field interference. When signatures are extracted sufficiently far from the model, a far-field propagation technique ( $\mathrm{sBOOM}$ ) is usable without correction because the acoustic sources appear to come from a single line.

Extracted CFD pressure signatures were requested for a complete cylinder aligned with freestream flow direction to enable a far-field correction methodology that accounts for a spanwise distribution of acoustic disturbances. ${ }^{30}$ However, ground signatures and ground loudness in this summary apply sBOOM directly to data extracted at distances where the near-field interference of spanwise disturbances is significant. The far-field correction is omitted because it eliminates some of the differences between submissions.

The scaled LM1021 wind tunnel model produces a louder PL than the flight vehicle it represents. The blade sting and the thickened boundary layers due to the lower wind tunnel Reynolds number distort the signature that is designed to be a quiet for the free-flight model at flight Reynolds number. CFD calculations on the full-scale free-flight model with far-field correction yield the PL in Table 6, which is quieter than the PL shown in this summary for the wind tunnel model without correction. The PL from two extraction distances varies by less than a tenth of a $\mathrm{dB}$ when near-field corrections are applied.

Table 6. Full-scale LM1021 far-field corrected CFD.

\begin{tabular}{rrrrrrr}
$R /(b / 2)$ extraction location & $\mathrm{PL}$ at $\phi=0$ & $\mathrm{PL}$ at $\phi=10$ & $\mathrm{PL}$ at $\phi=20$ & $\mathrm{PL}$ at $\phi=30$ & $\mathrm{PL}$ at $\phi=40$ & $\mathrm{PL}$ at $\phi=50$ \\
\hline 5.5 & 85.04 & 83.70 & 84.62 & 85.67 & 86.54 & 86.26 \\
10.9 & 85.00 & 83.67 & 84.52 & 85.52 & 86.46 & 86.28 \\
\hline
\end{tabular}

The $1 / 125$ scale model has a reference length of $L=22.4 \mathrm{in}$. and a half span of $b / 2=4.03 \mathrm{in}$. This yields a reference length to half span ratio of 5.6. The angle of attack is specified as $2.1^{\circ}$. The provided grid contains geometry that was rotated to the specified incidence, so it should be run at $0^{\circ}$ angle of attack. The workshop committee requested turbulent calculations at a Reynolds number per inch of 212,000. This request is slightly lower than the actual test Reynolds number per inch of 375,000 . This difference in Reynolds number had a negligible effect on the near-body signatures. ${ }^{28}$ The increase in Reynolds number resulted in improved measurement accuracy and productivity. ${ }^{28}$

The participants provided submissions containing entire half cylinders, where pressure signatures are extracted every $10^{\circ}$ at five radial locations, Table 7 . The submissions to this optional case are listed in Table 8. See section V.A for a description of the abbreviations that are common for the SEEB-ALR and LM1021 tables.

Table 7. LM1021 pressure signature extraction locations.

\begin{tabular}{rrrl}
$H$ & $H / L$ & $H /(b / 2)$ & $\phi$ \\
\hline 19.7 in. & 1.1 & 4.9 & every $10^{\circ}$ from $0^{\circ}$ to $180^{\circ}$ \\
31.8 in. & 1.8 & 7.9 & every $10^{\circ}$ from $0^{\circ}$ to $180^{\circ}$ \\
42.0 in. & 2.3 & 10.4 & every $10^{\circ}$ from $0^{\circ}$ to $180^{\circ}$ \\
70.0 in. & 3.9 & 17.4 & every $10^{\circ}$ from $0^{\circ}$ to $180^{\circ}$ \\
100.7 in. & 5.6 & 25.0 & every $10^{\circ}$ from $0^{\circ}$ to $180^{\circ}$ \\
\hline
\end{tabular}

Table 8. LM1021 submissions.

\begin{tabular}{rlllllr} 
Submission & Group & Method & Physics & Type & Grid & Cont. Vol. \\
\hline 1 & A & FUN3D & SA & Tet & WS & $2,395,158$ \\
2 & B & FUN3D & SA & Tet & VGRID & $27,000,000$ \\
3 & C & USM3D & Laminar & Tet & WS & $13,737,358$ \\
4 & C & USM3D & SA & Tet & WS & $13,737,358$ \\
5 & C & USM3D & SST & Tet & WS & $13,737,358$ \\
6 & C & USM3D & SA & Tet & PW/MCAP & $65,400,000$ \\
$7^{*}$ & E & Eugenie & Euler & Tet & Fixed & $13,737,358$ \\
8 & M & Cart3D & Euler & Cut Cell & Output & $57,000,000$ \\
9 & O & CartOver & Euler & Hybrid & Fixed & $51,018,868$ \\
10 & Q & CFD++ & SA & Tet & WS & $13,737,358$ \\
$11^{*}$ & Q & CFD++ & SA & Tet & Inflate & $23,463,802$ \\
\hline
\end{tabular}

* Updated or submitted after the workshop. 


\section{Near-Field Wind Tunnel Measurements}

Spatial and temporal variation in wind tunnel test section Mach, static pressure, and flow direction complicates near-field pressure measurements, because these noise sources are of the same magnitude as the signals the test is intended to measure. These disturbances have been known for decades ${ }^{31}$ and are now better understood with the availability of time ${ }^{32}$ and space ${ }^{33}$ resolved measurements. Typical spatial disturbances are clearly illustrated by distortion in measurements of the flattop portion of the SEEB-ALR signature. ${ }^{34}$

A better understanding of the disturbances present in all supersonic wind tunnels ${ }^{35}$ has led to a spatial averaging technique ${ }^{34,36}$ used in conjunction with a pressure measurement rail. ${ }^{37}$ The evaluation and development of different rail configurations was also used to quantify the effect of humidity on wind tunnel measurements. ${ }^{38}$

The experimental signatures in this summary were the result of the spatial averaging technique, which provides mean signatures and variation among the ensemble of measurements used to compute the mean signature. This variation among the ensemble of measurements is expressed as a standard deviation at each $x$ location. This spatial averaging technique was complicated by spatial variation in flow angularity ${ }^{39}$ that caused variation in angle of attack and lift as the model was translated. ${ }^{28}$ Bretl and Walker ${ }^{40}$ propagated uncertainty derived from the near-field measurement variation and estimated uncertainties in the range of 0.5 to $4 \mathrm{~dB}$ uncertainty in PL, depending on how the near-field variation was represented.

Wind tunnel models for off-body near-field pressure measurements are generally smaller than force and moment models. These smaller models can amplify Reynolds number sensitivity and require characterization of the boundary layer state to increase the fidelity of simulation. ${ }^{6}$ This is critical for models with small details (e.g. nacelle boundary layer diverters, ${ }^{12}$ nacelles in the wake of wind tunnel mounting hardware ${ }^{6}$ ). Boundary layer trip devices further complicate measurement and simulation because they are directly observed in nearbody measurements and affect boundary layer state, which impacts aft-body signatures. ${ }^{41,42}$

A summary of the experimental measurements used in the workshop was provided by Cliff et al. ${ }^{28}$ Morgenstern, Buonanno, and Marconi ${ }^{6}$ provided additional details of the LM1021 test. Makino and Noguchi ${ }^{5}$ performed measurements on an independently constructed DWB model.

\section{SEEB-ALR Analysis}

The analysis for the SEEB begins by examining the submissions at the closest extraction distance and these signatures propagated to the ground. The variation in PL and validation metric measures is examined with grid refinement and the statistics of these measures is examined. Finally, signatures that have statistically significant measures are examined. This same analysis process will be repeated for the DWB.

\section{VII.A. SEEB-ALR Near-Body Signatures}

The submitted signatures from Table 3 are shown as red lines in Fig. 8. The wind tunnel data has been shifted in $x$ to align the bow shock to the middle of the participants. The mean of two wind tunnel test series are shown as the solid black lines. One standard deviation of the measurement ensemble variation is shown as the dashed black lines for each test series. All 64 submissions are shown in Fig. 8(a) and 30 submissions (excluding the coarser grids of the uniform refinement study) are shown in Fig. 8(b). The positions of the secondary nose shock, expansion, and the tail shock are predicted by the majority of the participants. The flattop pressure level is predicted within a standard deviation of the wind tunnel measurements, $28<x<37$. The participant pressure signatures in this flattop region are at or above the lower pressure wind tunnel mean. The submissions are lower than the wind tunnel mean expansion pressures and slightly below one standard deviation of the wind tunnel measurements, $39<x<43$. Eliminating the coarser uniformly refined grids does not appear to reduce the scatter of the results, Fig. 8(b).

Some of the signatures provided do not have a point of inflection at the secondary nose shock at $x=28$, Fig. 9. Participants $\mathrm{C}$ and $\mathrm{D}$ also provided signatures on the workshop provided tetrahedral grids that exhibited a secondary nose peak (not shown). Participant B only provided 15B, which used a custom grid and did not show the secondary shock. This missing secondary nose peak is likely due to geometry or grid differences, because the same CFD method using different grids showed the secondary peak. A possible explanation is the use of as-designed geometry which has as slightly different nose shape. ${ }^{28}$ As-built geometry was provided for the workshop. 


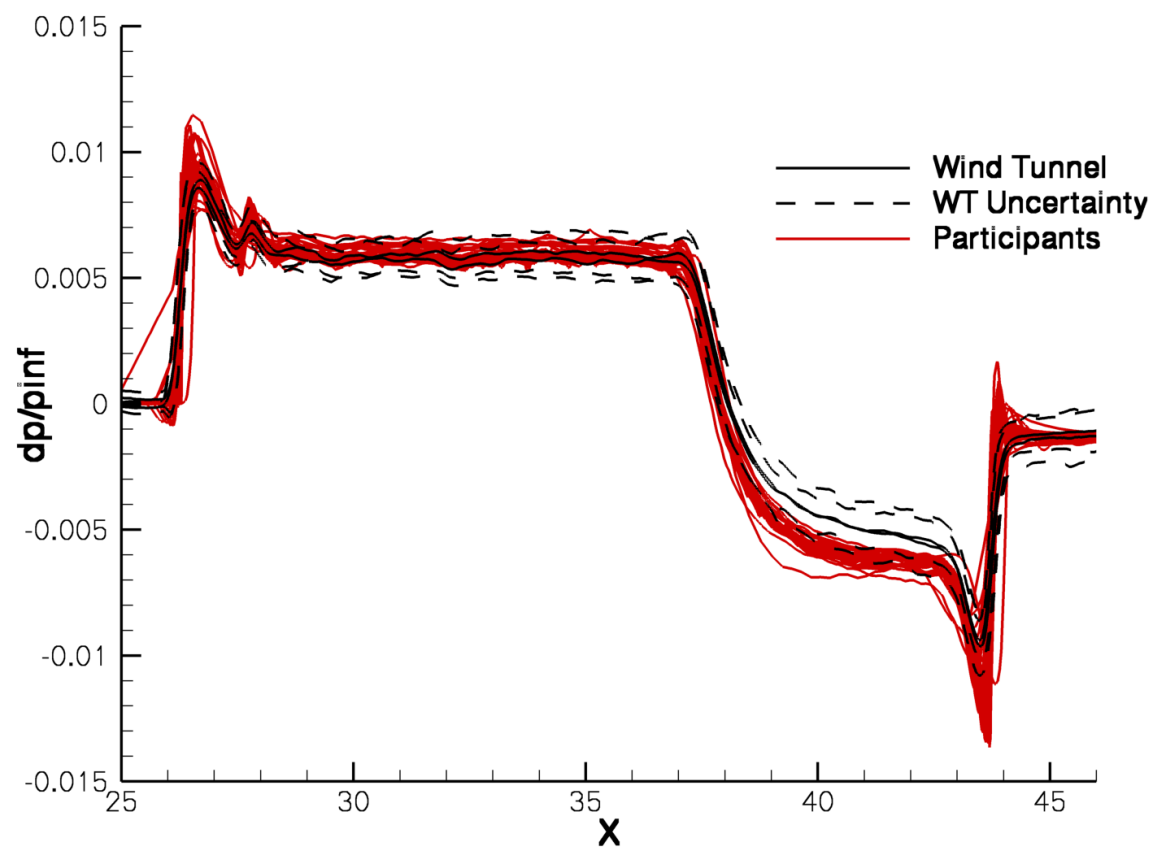

(a) All submissions.

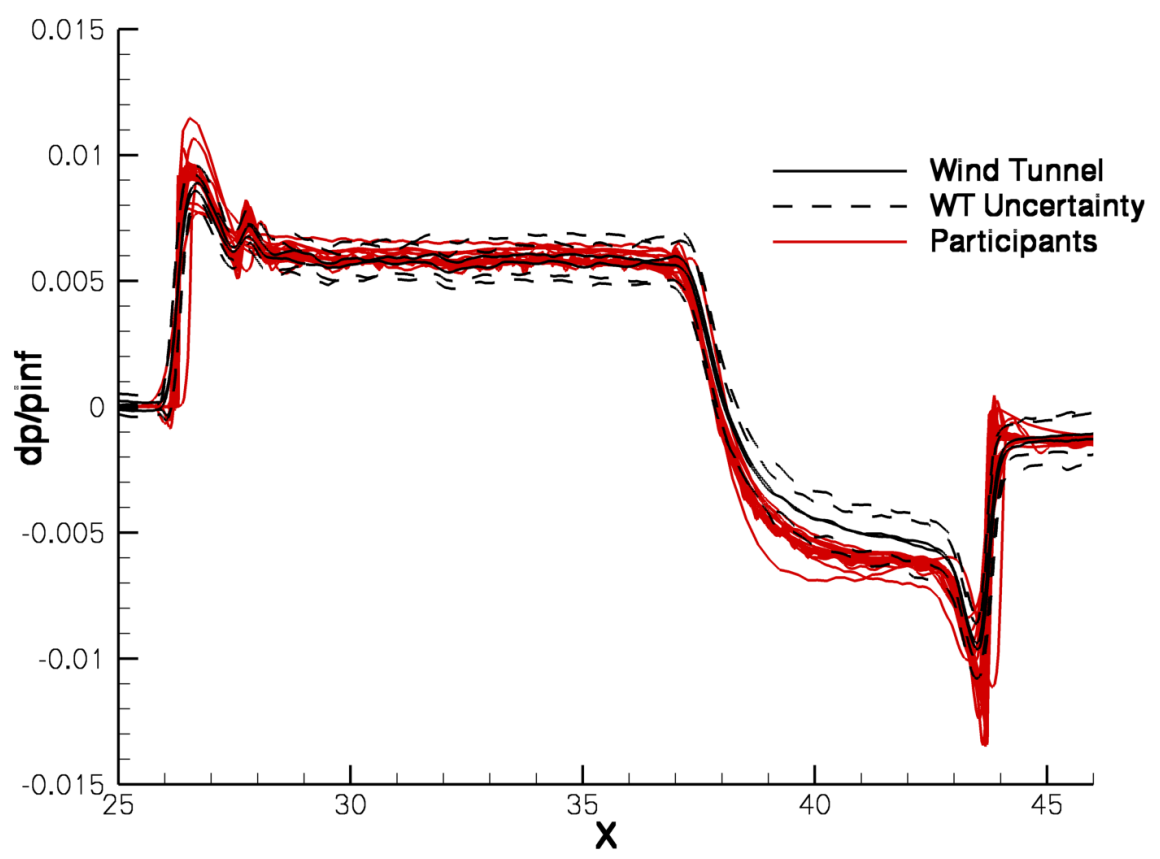

(b) Fine-grid submissions, excluding coarser uniform grids.

Figure 8. SEEB-ALR near-body pressure signatures at $H=21.2 \mathrm{in}$. 


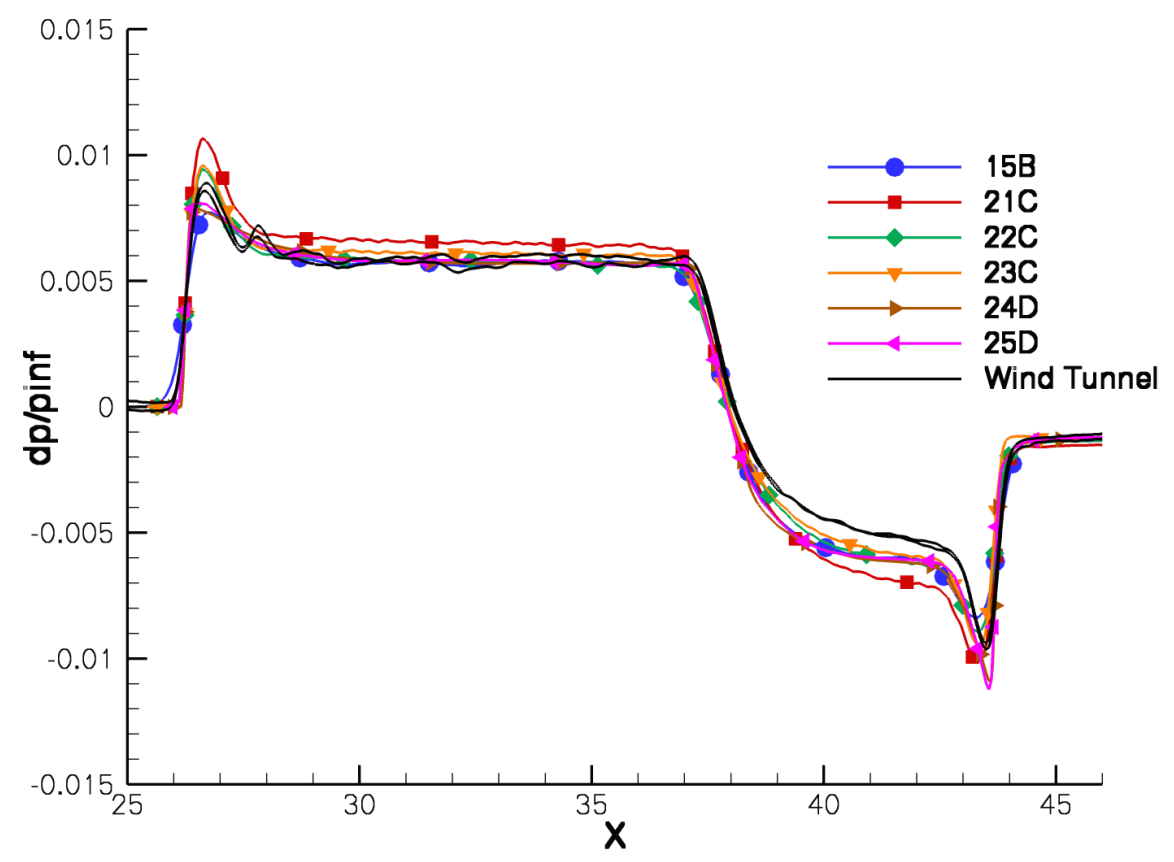

Figure 9. SEEB-ALR submissions exhibiting a single peak at $H=21.2 \mathrm{in}$.

Submissions based on Euler calculations were requested for this case, but some participants submitted signatures extracted from turbulent RANS solutions. Figure 10 shows the turbulent submissions for the SEEB-ALR. The Spalart-Allmaras (SA) turbulence model was used for the 23C, 59Q, 61R, and 64S submissions. The Mentor Shear Stress Transport (SST) turbulence model was used for the 63R submission. These signatures (except for submission 23C) showed the secondary nose shock. As a group, the turbulent signatures had a slightly higher pressure in the aft expansion (closer to the mean wind tunnel measurements) than the Euler signatures, $39<x<43$.

\section{VII.B. SEEB-ALR Ground Signatures}

All of the near-body SEEB submissions and the mean wind tunnel measurements at $\mathrm{H}=21.2 \mathrm{in}$. are propagated to the ground, Fig. 11(a). The propagation is performed with the method described in section II.B. The time axis of the ground signatures have been shifted so that all the initial pressure rises occur at $100 \mathrm{~ms}$. The two mean wind tunnel measurement have slightly different flattop pressure levels, but nearly identical expansions and aft shock positions. The ensemble of submitted signatures produce ground signatures that are at or higher than the wind tunnel mean for the flattop and lower than the wind tunnel mean for the expansion. This is the same trend that is seen in the near-body signatures, Fig. 8(a).

The coarser submissions in uniform grid refinement families are excluded in Fig. 11(b). The ensemble pressure scatter is not dramatically reduced by excluding the coarser grids. The is the same trend exhibited by near-body signatures in Fig. 8(b).

\section{VII.C. SEEB-ALR Uniform Refinement}

Ten sets of uniformly refined grids from eight of the nineteen participants were submitted for the SEEB-ALR. The participants were instructed to perform a calculation on the finest committee provided grid that they had the ability to run, and as many coarser grids in that family as possible. Some participants were not able to comply due to time constraints, and some solvers were not compatible with the provided grid types (e.g., Cartesian cut-cell methods, multiblock structured methods that can not handle singularities). 


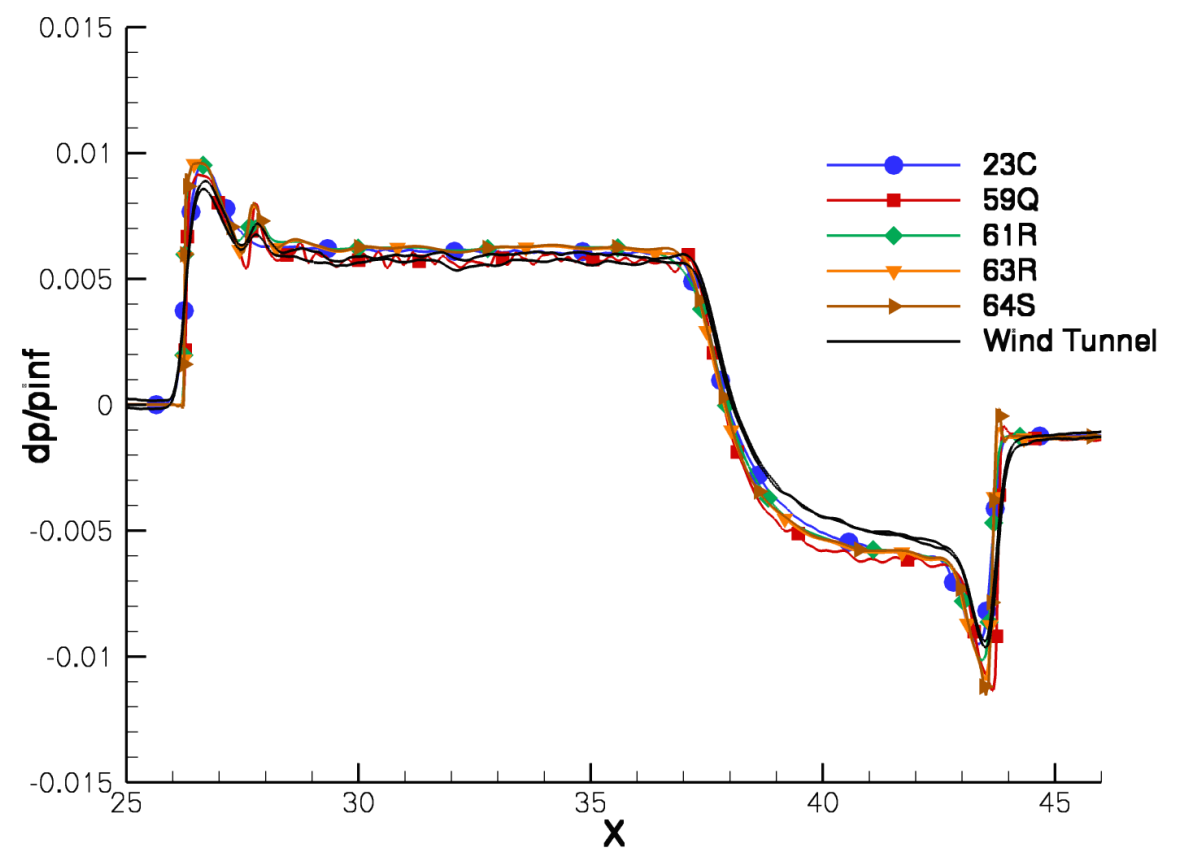

Figure 10. SEEB-ALR turbulent CFD submissions at $H=21.2 \mathrm{in}$.

The PL of the propagated ground signatures is shown in Fig. 12(a). PL is calculated with the method described in section II.A. The characteristic grid spacing $h$ is estimated as the number of control volumes to the negative one third power. The unstructured grids have an approximate doubling of control volumes between grids in the family. This results in successive unstructured grids with an approximate cube root of a half factor between successive $h$ values. The structured grid has a factor of eight increase in control volumes (with a halving of $h$ ). Extrapolating the submission series to $h=0$ (infinitely refined grid) yields approximately 91.7 PL (dB). There are no obvious trends for structured, mixed-element, or purely-tetrahedral grid submissions.

The validation metric $V$ is a measure of the difference between the submitted signatures and the mean wind tunnel measurement, Fig. 12(b). It is calculated with the method described in section II.C. This metric is expected to trend to a non-zero value with grid refinement because of the inherent differences between the mean wind tunnel measurement and submitted signatures (e.g. incomplete modeling of the physics with the Euler equations, rounding of the wind tunnel measurements due to averaging). Each SEEB-ALR uniform refinement series extrapolates to $V \approx 0.0006$ at $h=0$ (infinitely refined grid).

\section{VII.D. SEEB-ALR Statistics}

The statistical approach of section III is used to compute the median and limits of the entire population of PL propagated from $H=21.1$ in. in Fig. 13(a), which includes coarser uniformly refined grids by the same participant. The median is near 91.7 PL (dB), which agrees with the uniform grid refinement extrapolation. The two diamond symbols are the PL of both wind tunnel measurement means. The wind tunnel PL values are both slightly lower than the mean of the submissions, but within the limits of the participant statistics. All outliers have a larger PL than the upper limit.

The fine grid submissions are shown Fig. 13(b) by removing the the coarser grids in the uniform grid refinement study from Fig. 13(a). The median and limit statistics are very similar for this smaller (but more independent) sample of the population. The outliers from participant E in Fig. 13(a) are on coarser grids; the finest grid submitted by participant E in Fig. 13(b) is not statistically significant among other fine-grid results. Signatures from the $21 \mathrm{C}$ and $43 \mathrm{I}$ submissions have a louder PL than the limits. Details of these near body signatures are examined in section VII.E. 


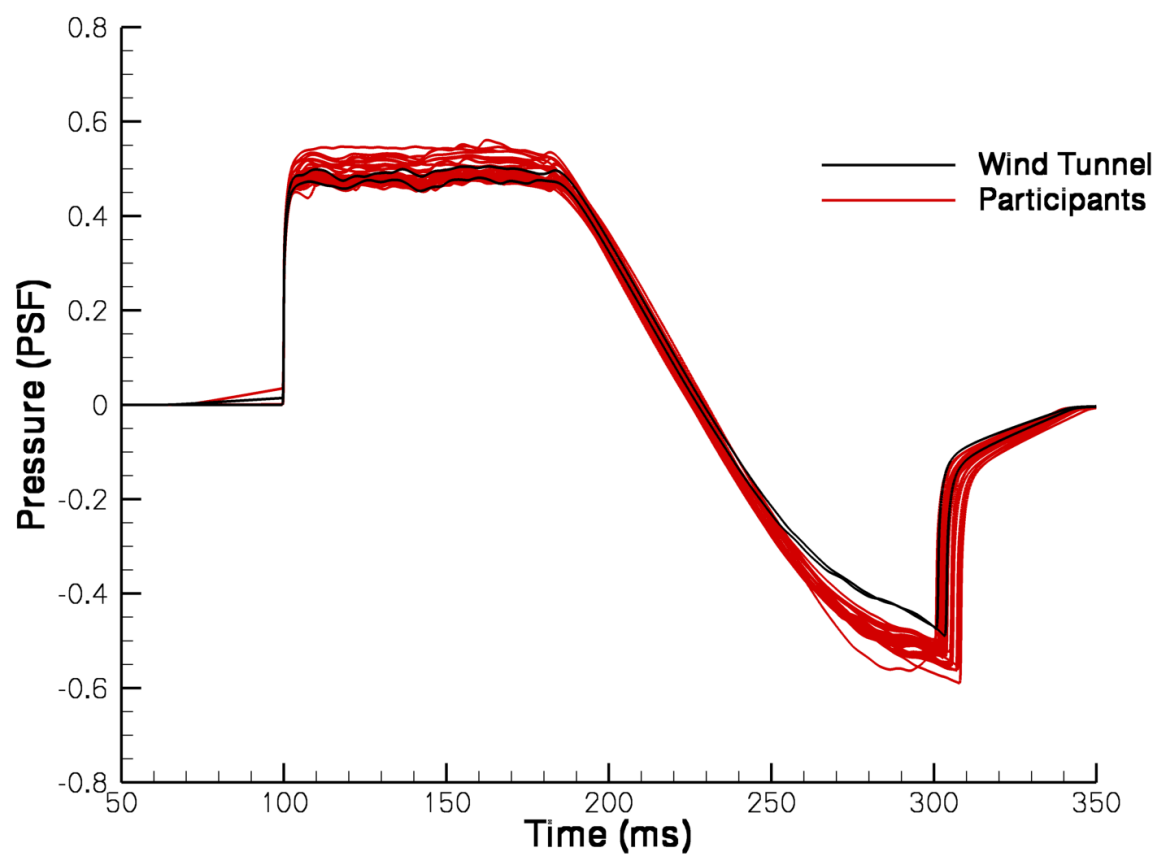

(a) All submissions.

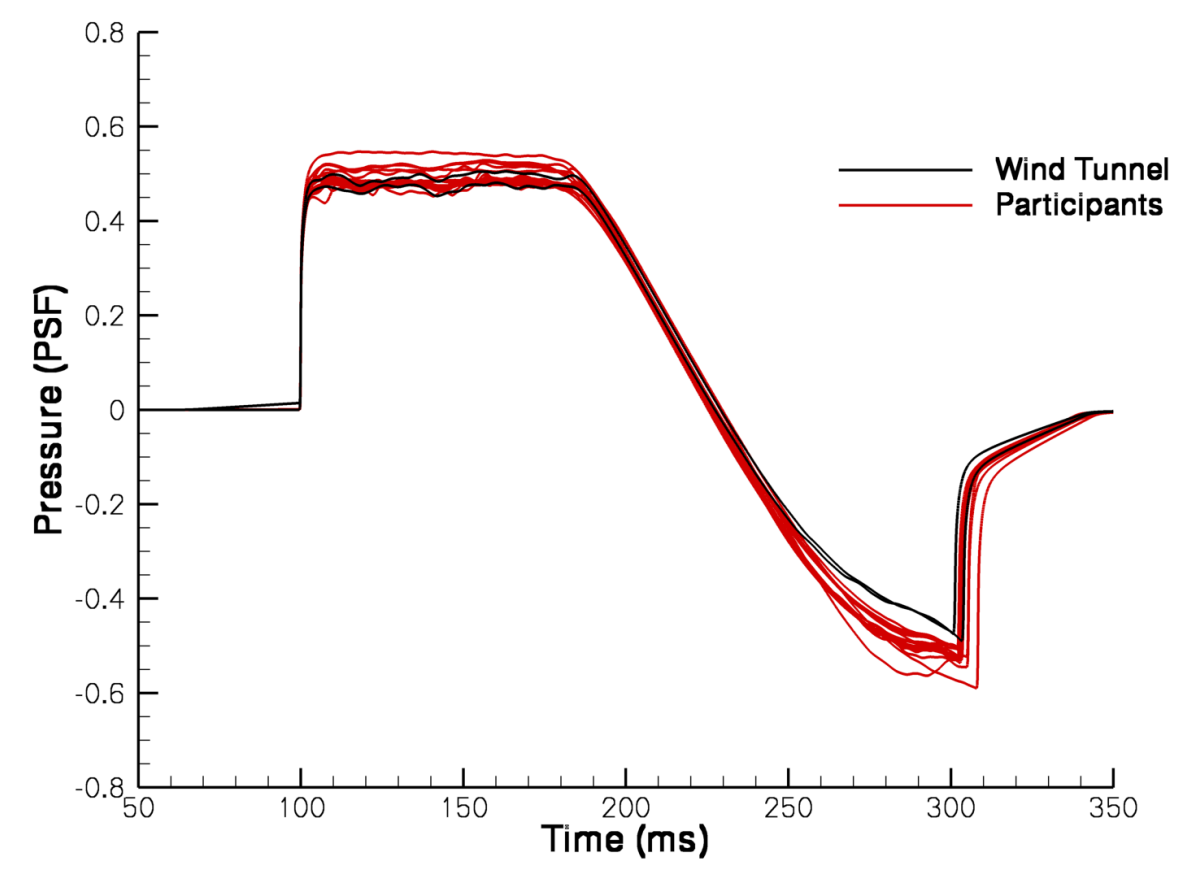

(b) Fine-grid submissions, excluding coarser uniform grids.

Figure 11. SEEB-ALR propagated ground signatures from $H=21.2$ in. 


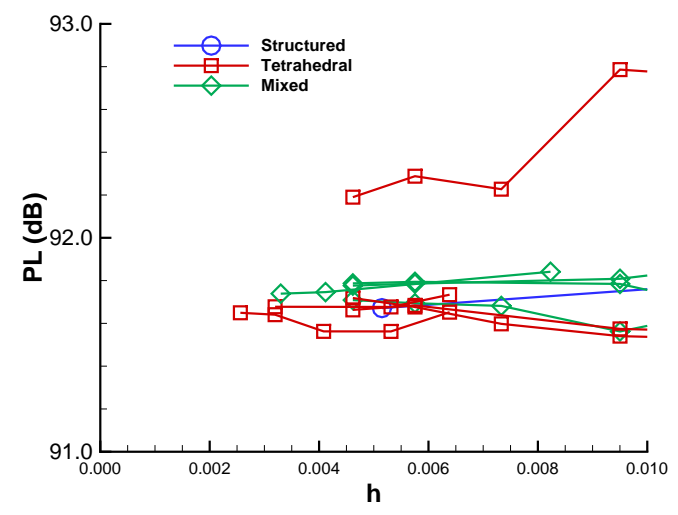

(a) PL propagated from $H=21.2$ in.

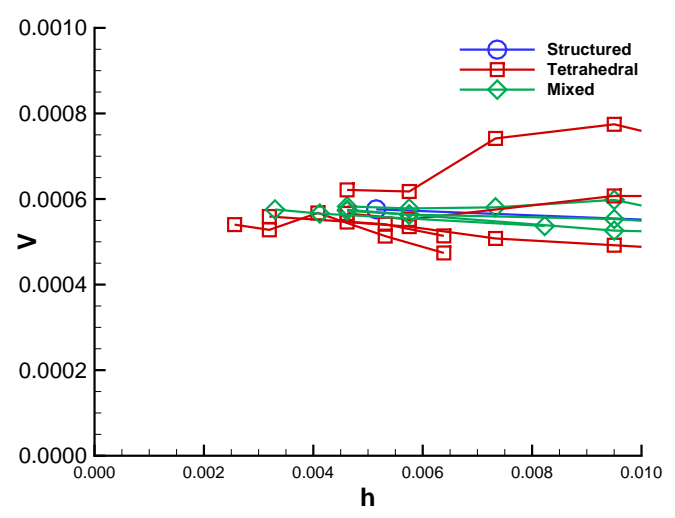

(b) $V$ at $H=21.2$ in.

Figure 12. SEEB-ALR uniform grid refinement.

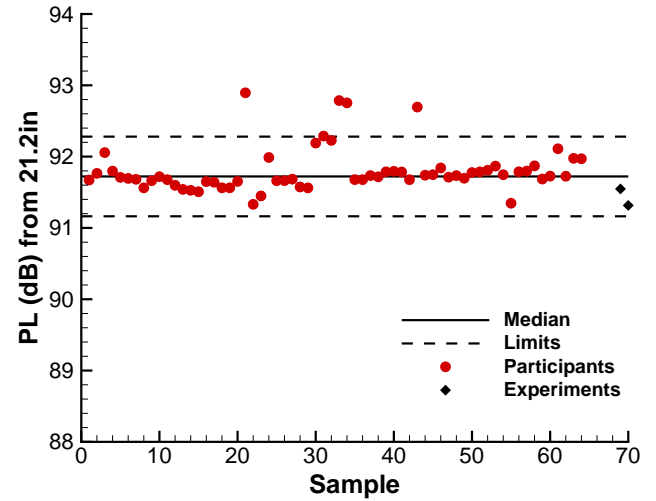

(a) All submissions. 21C, 31E, 33E, 34E, and 43I are above the limits.

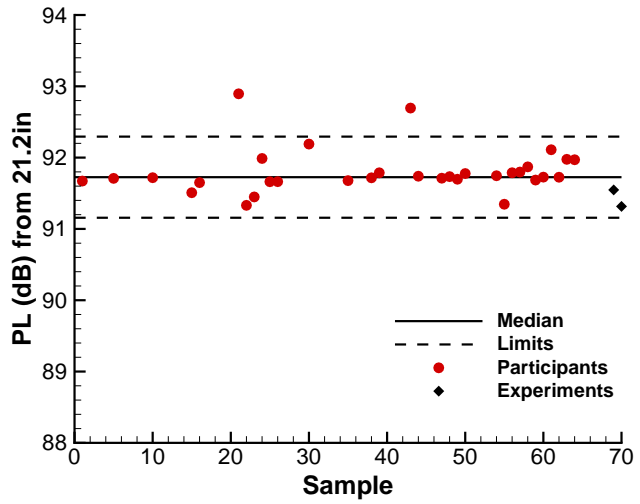

(b) Fine-grid submissions, excluding coarser uniform grids. $21 \mathrm{C}$ and $43 \mathrm{I}$ are above the limits.

Figure 13. SEEB-ALR PL statistics propagated from $H=21.2$ in. 
The PL from both of the near body signature extraction locations are shown in Fig. 14(a). The wind tunnel PL is only propagated from $H=21.2 \mathrm{in}$. This slender, nominally axisymmetric body is expected to have very similar propagated PL values for these locations at 1.2 and 2.4 body lengths. Significant differences in PL from these two locations would indicate changes to the signature within the CFD domain.

The differences between PL at $H=42.0$ in. and $H=21.2$ in. are shown in Fig. 14(b). All the outliers have a lower PL at the larger extraction location. Submission 25D is the only submission not involving a coarser grid in a uniform grid family.

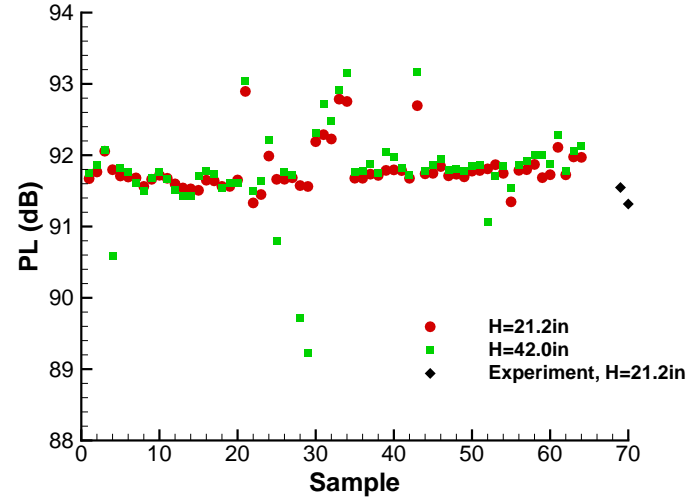

(a) PL propagated from $H=21.2$ in. and $H=42.0$ in.

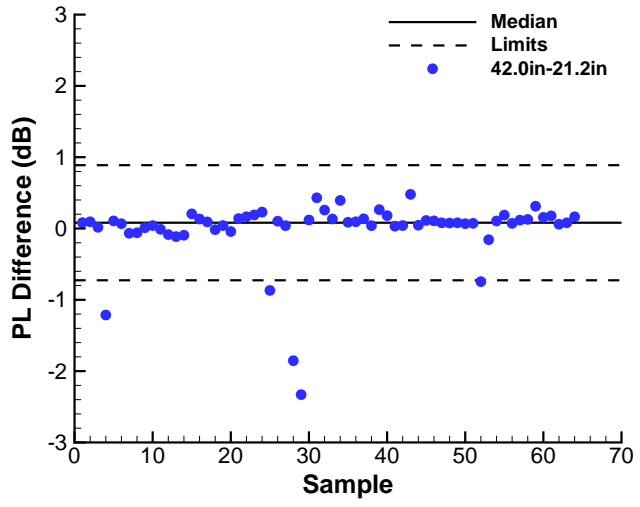

(b) PL difference between $H=21.2 \mathrm{in}$. and $H=42.0 \mathrm{in}$. $4 \mathrm{~A}, 25 \mathrm{D}, 28 \mathrm{D}, 29 \mathrm{D}$, and $52 \mathrm{~L}$ are below the limits.

Figure 14. SEEB-ALR PL statistics propagated from two near-body distances.

\section{VII.E. SEEB-ALR Outliers}

Submissions 21C and 43I near-field signatures are shown in Fig. 15. These submissions have a louder PL than the limits in Fig. 13(b). The 21C Euler custom grid submission has a higher flattop pressure than the wind tunnel mean and signatures by the same participant using the same code on the committee-provide grids. Submission 43I is a linear method. This submission exhibits many of the small shocks in the measurement mean flattop region, but has a unique expansion shape, which exibits a much stronger expansion and recovery slightly before the aft shock.

Submission 25D is the only submission (not a coarse grid in a uniform refinement family) that is outside of the limits in Fig. 14(b). The 25D near-body pressure signatures at $H=21.2 \mathrm{in}$. and $H=42.0$ in. are scaled by $\sqrt{H / L}$ and shifted in $x$ by the $H \beta$, Fig. 16. After this transformation, the two signatures should over plot because both extraction locations are sufficiently far from this axisymmetric body. However, the transformed $H=42.0$ in. signature has a lower pressure value than the transformed $H=21.2$ in. signature.

\section{DWB Analysis}

The same analysis steps applied to the SEEB-ALR are now applied to the second required workshop configuration, the DWB. It is slightly more complex than the SEEB-ALR and provides a slight variation in on- and off-track near-field signature because the wing has different impact on the signature at different $\phi$ locations.

\section{VIII.A. DWB Near-Body Signatures}

The submitted signatures from Table 5 are shown as red lines in Fig. 17 for $H=24.8$ in. and $\phi=0^{\circ}$ (centerline). The mean of wind tunnel measurement ensemble is shown as the solid black line. One standard deviation of measurement ensemble variation is shown as the dashed black line. The wind tunnel data has been shifted in $x$ to align the strong expansion to the median of the participants. All 60 submissions are shown in Fig. 17(a) and 31 submissions (excluding the coarser grids of the uniform refinement study) are shown in Fig. 17(b). Without the submission on coarser grids, the positions of the shocks and expansions 


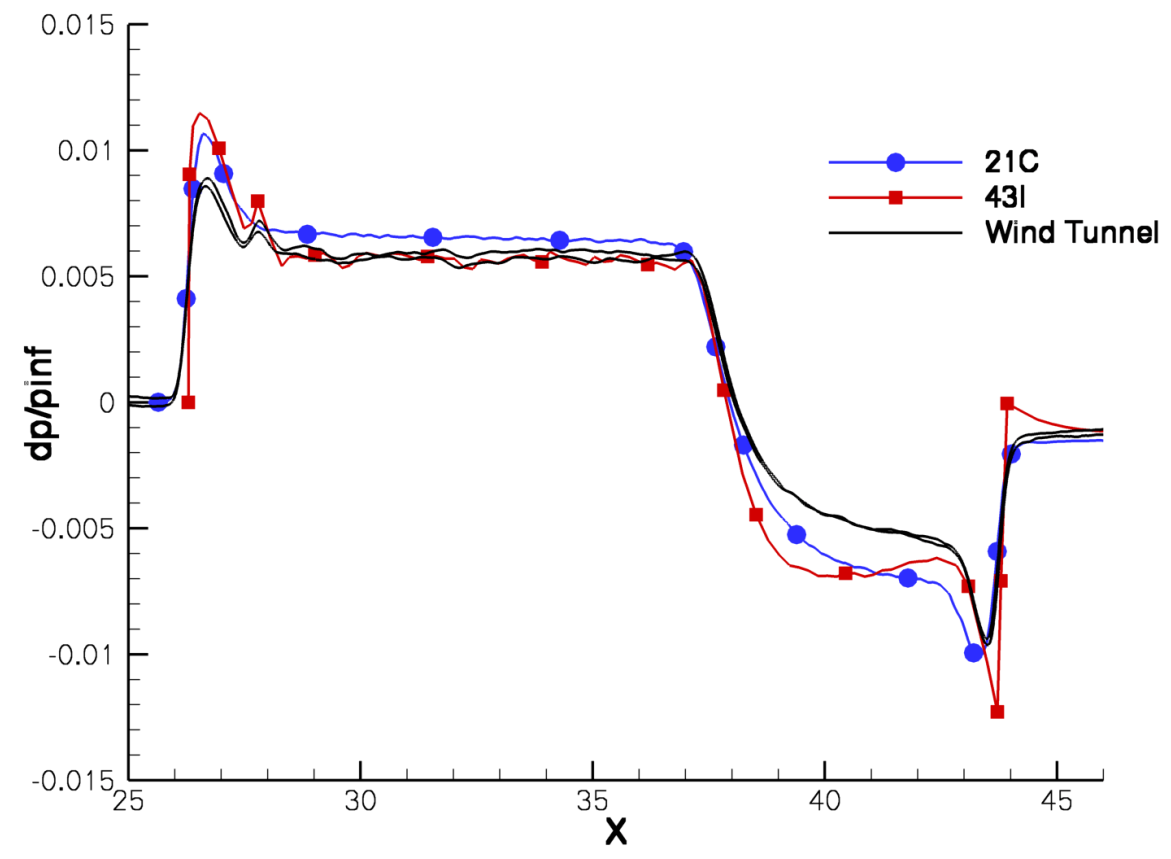

Figure 15. SEEB-ALR fine-grid PL outliers at $H=21.2$ in.

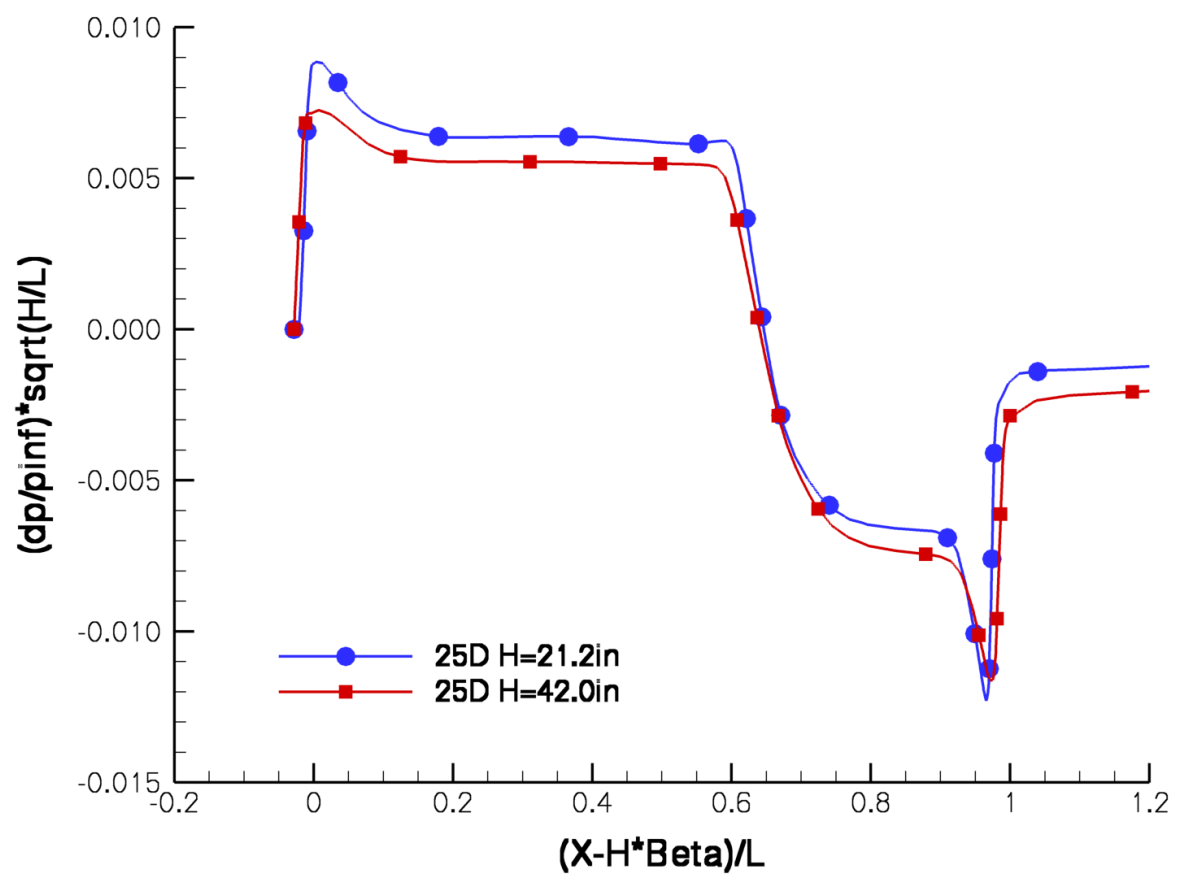

Figure 16. SEEB-ALR fine-grid PL difference outlier. 
are predicted by the majority of the participants. The first two shocks are due to the nose and the wing. The series of small shocks starting at $X=1.08 \mathrm{~m}$ are due to the expansion and re-compression of the base of the model. The pressure wave centered at $X=1.18 \mathrm{~m}$, only seen on coarse grids of Fig. 17(a), is due to poor wind tunnel sting discretization on the coarser committee provided grids. ${ }^{27}$ There is a large spread on the predicted level of overshoots and undershoots for the shocks. There is a small expansion in the middle of the aft wing shock that is predicted by the participants at $X=1.05 \mathrm{~m}$ but not shown in the mean wind tunnel measurements.

\section{VIII.B. DWB Ground Signatures}

The submitted signatures are propagated to the ground from $H=24.8 \mathrm{in}$. and $\phi=0^{\circ}$ in Fig. 17. All submissions are shown in Fig. 18(a) and the coarser grids of the uniform refinement study are excluded in Fig. 18(b). The propagated submissions are shown in red and the propagated wind tunnel median is shown in black. The time axis of the ground signatures have been shifted so that all the initial pressure rises occur at $100 \mathrm{~ms}$. The large variation in overshoots and undershoots and the complex signature due the the base flow seen in the near field have been filtered by the propagation process. The location of the middle and aft shocks shows more variation than the expansion slopes. This change in shock positions results in different minimum and maximum pressures of the main expansion.

\section{VIII.C. DWB Uniform Refinement}

Ten sets of uniformly refined grid submissions from eight participants are available for the DWB. The PL of the propagated ground signatures is shown in Fig. 19. The characteristic grid spacing $h$ is estimated as the number of control volumes to the negative one third power. The DWB, Fig. 19, shows more variation than the SEEB-ALR, Fig. 12(a), when extrapolating the finest two grids from each series to zero $h$ (infinitely refined grid). This extrapolation to zero $h$ results in PL values between 95.2 and $95.6 \mathrm{~dB}$.

The grid convergence of the validation metric is examined in Fig. 20. The metric for the entire signature at $H=24.8 \mathrm{in}$. and $\phi=0^{\circ}$ is shown in Fig. 20(a). When each $V$ series is extrapolated to zero $h$ (infinitely refined grid) there is less agreement of the extrapolated $V$ than than SEEB-ALR $V$ or DWB PL. To isolate the source of the larger spread in extrapolated values, the validation metric integral, Eq. (1), extent is limited to the forward portion of the signature to isolate it from the the impact of the base flow region. The whole extent is $X=[0.8,1.25]$ and the forward extent is $X=[0.8,1.0]$. Restricting $V$ to the forward portion of the signature did not reduce the scatter of the results, indicating that the base flow is not the only contributor to the scatter. The overshoots and undershoots of the strong shocks may be adversely impacting convergence of $V$ with grid refinement.

\section{VIII.D. DWB Statistics}

The DWB PL propagated from $H=24.8$ in. is shown for the centerline (Fig. 21), $30^{\circ}$ off-track (Fig. 22), $60^{\circ}$ off-track (Fig. 23), and $90^{\circ}$ off-track (Fig. 24). The DWB sBOOM atmospheric propagation is performed directly down for each extracted signature, so every signature experiences the same atmospheric model. This approach is not the process commonly used to compute boom "carpets" where each signature is propagated at the same oblique angle where it is extracted. Using the same atmospheric model for all DWB signatures facilitates comparison between near-field signatures extracted at different $\phi$-angles, including $90^{\circ}$ off-track which would not reach the ground in a standard atmosphere. The sample median (solid black lines) and the propagated mean wind tunnel measurement (black diamonds) compare favorably for all $\phi$. Both the median participant and experimental PL values and increase with $\phi$. This is because the wing shock strength and spatial separation between the trailing edge wing shock and the base region shocks change with $\phi$.

All submissions are plotted in each (a) sub-figure and the coarse grids of the uniform refinement series are excluded from each (b) sub-figure. The sample median (solid lines) is insensitive to excluding the coarser refined grids, but the limits (dashed lines) grow slightly by approximately a tenth of a dB. This increase in the limits does not eliminate any fine-grid submissions that are outliers of all submissions. All DWB outliers are below the limits. Submission 4A is the coarsest DWB grid in the workshop with 19.8 thousand cells/control volumes. Submission 33G is an outlier for all four $\phi$-angles. Participant G indicates in their workshop presentation that the DWB structured grid wing tip singularity is an issue that negatively impacted the CFD tool. Submission 53N is an outlier at and above $\phi=30^{\circ}$. This submission uses a manually refined, 


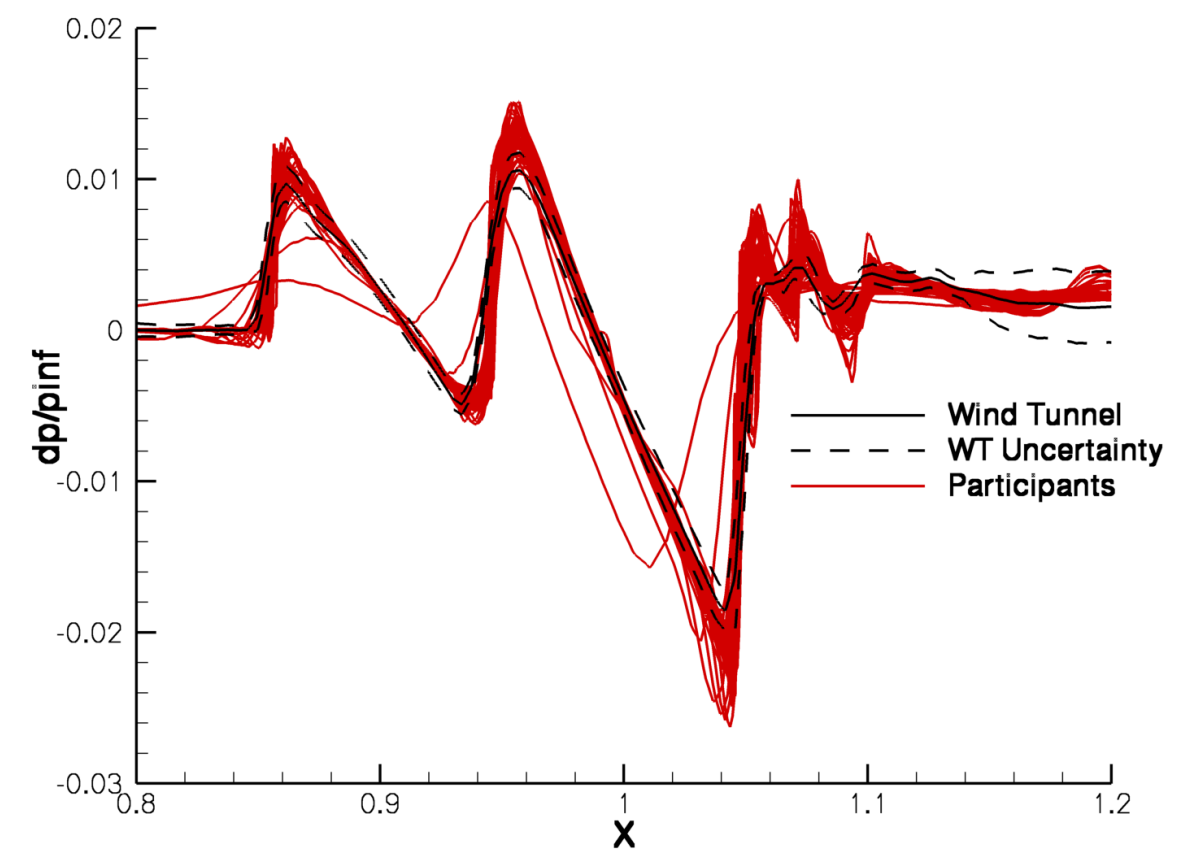

(a) All submissions.

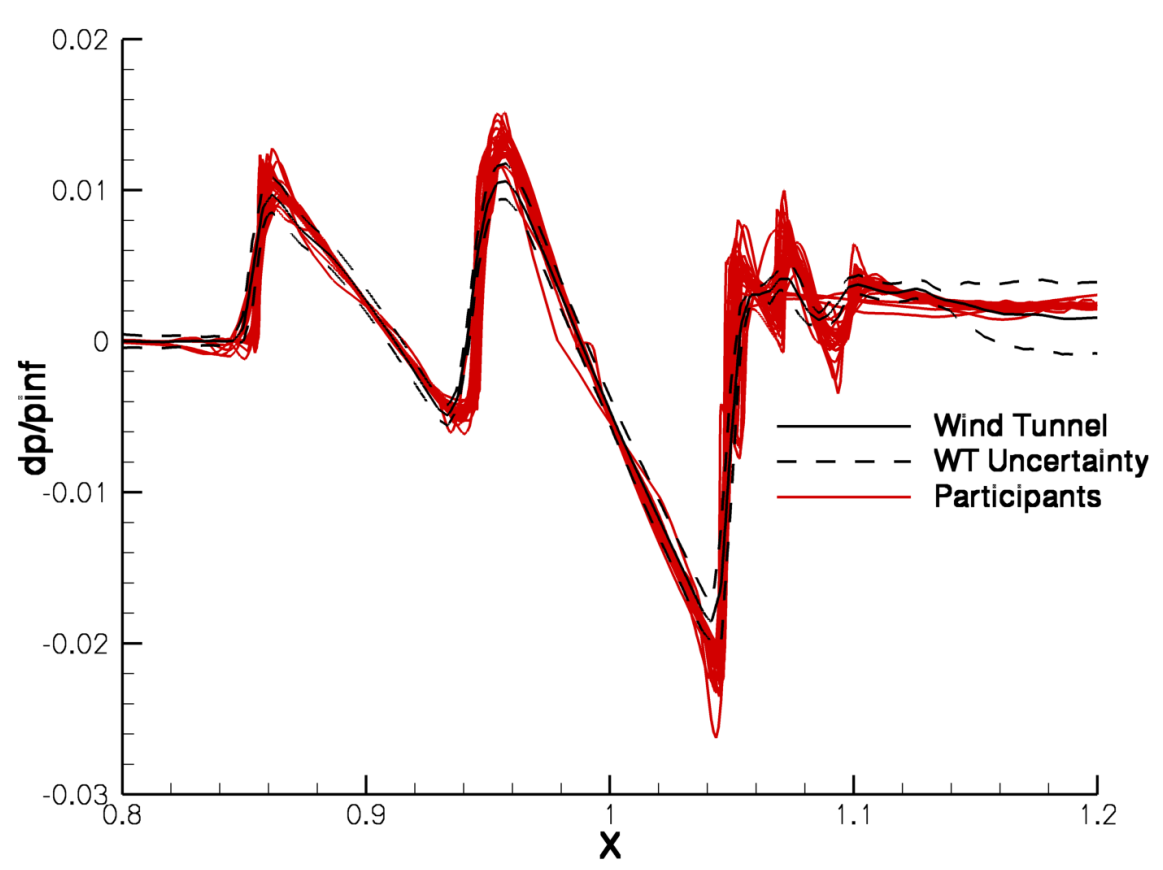

(b) Fine-grid submissions, excluding coarser uniform grids.

Figure 17. DWB near-body pressure signatures at $H=24.8$ and $\phi=0^{\circ}$. 


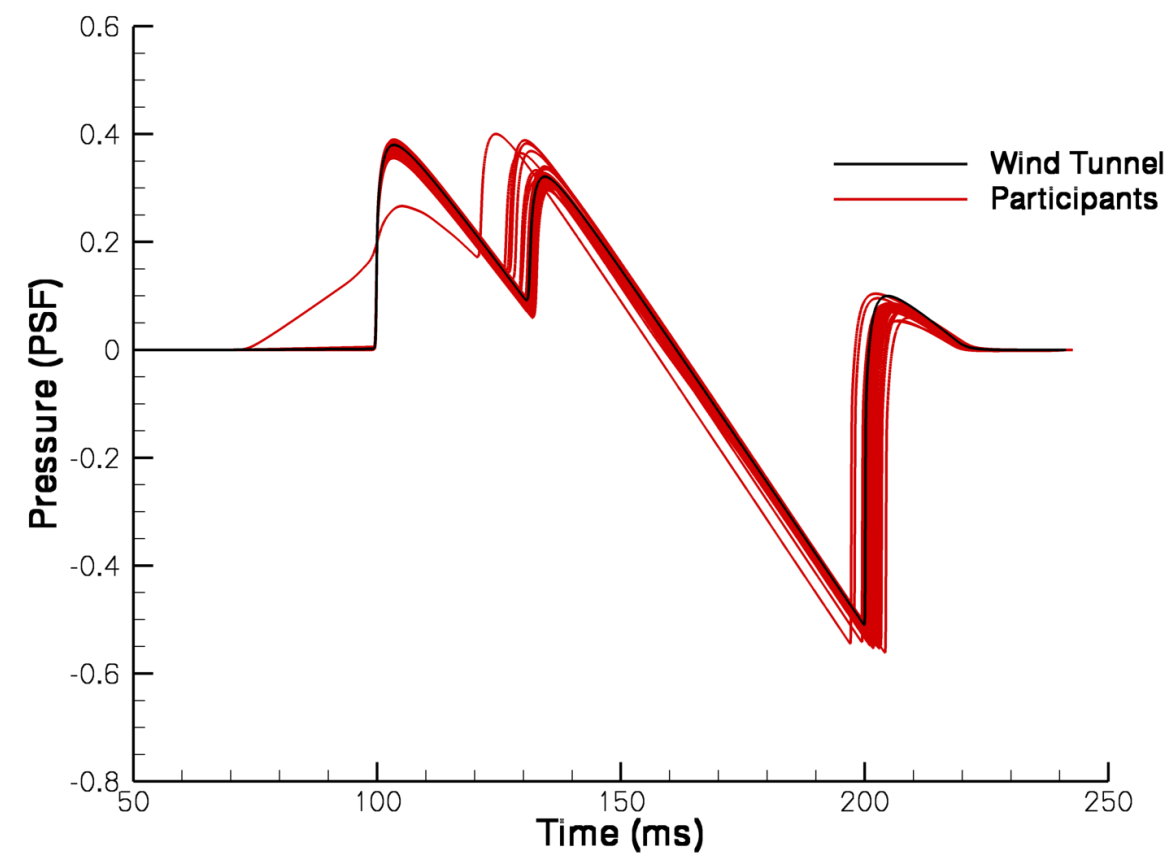

(a) All submissions.

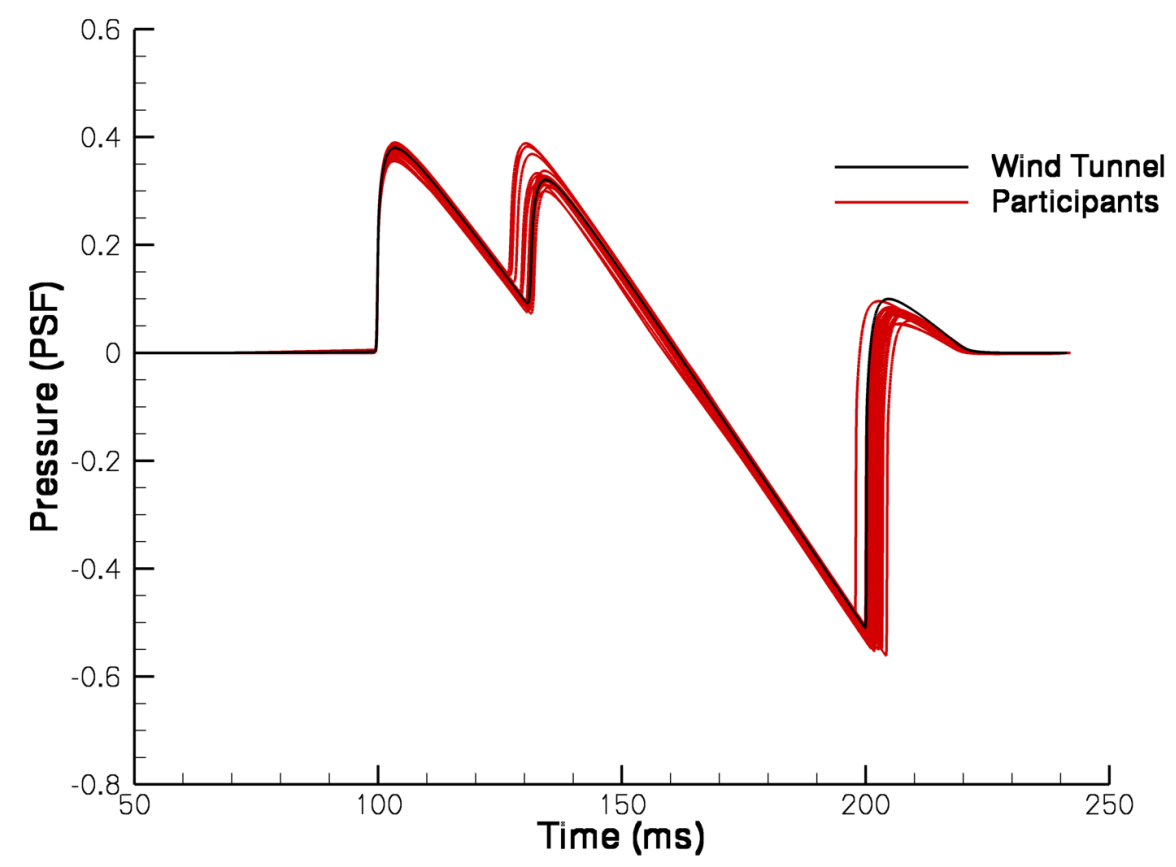

(b) Fine-grid submissions, excluding coarser uniform grids.

Figure 18. DWB propagated ground signatures from $H=24.8$ and $\phi=0^{\circ}$. 


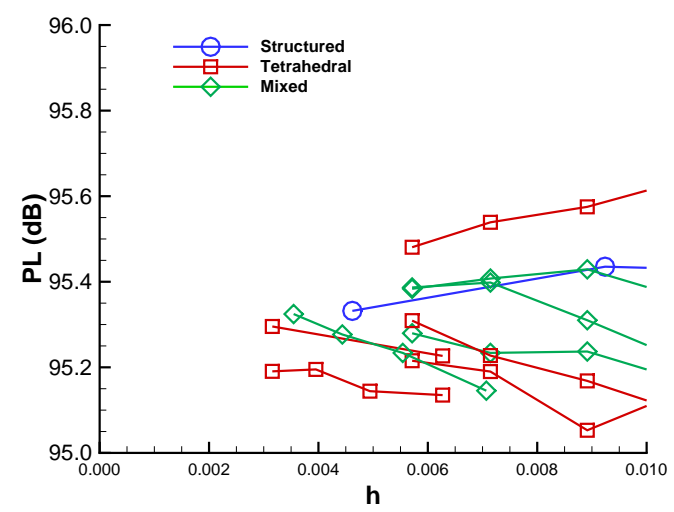

Figure 19. DWB PL uniform grid refinement propagated from $H=24.8$ and $\phi=0^{\circ}$.

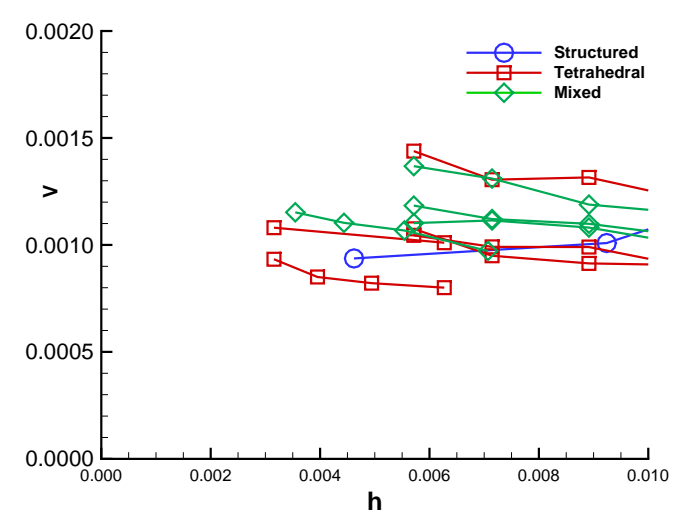

(a) The entire signature.

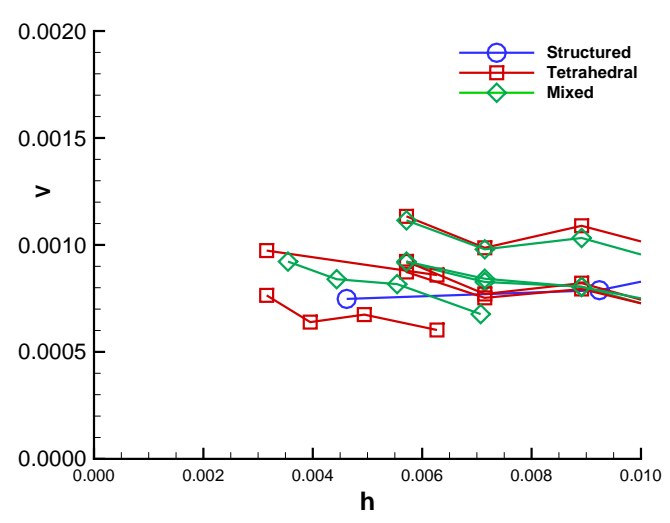

(b) The forward portion of the signature.

Figure 20. DWB validation metric uniform grid refinement at $H=24.8$ and $\phi=0^{\circ}$.

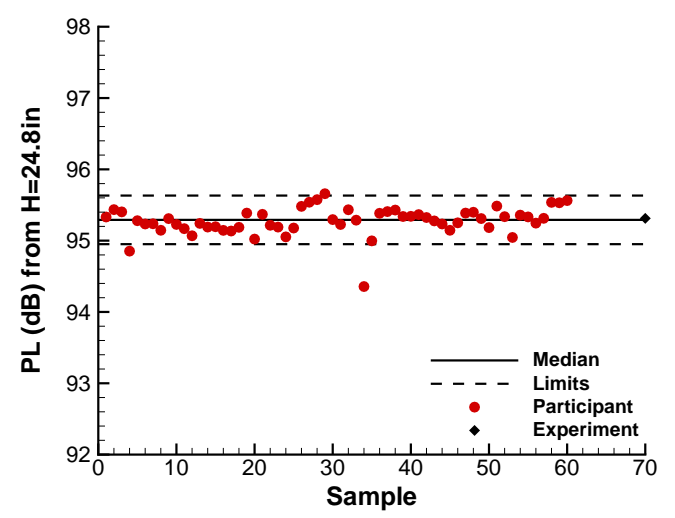

(a) All submissions, $4 \mathrm{~A}$ and $34 \mathrm{H}$ are below the limits.

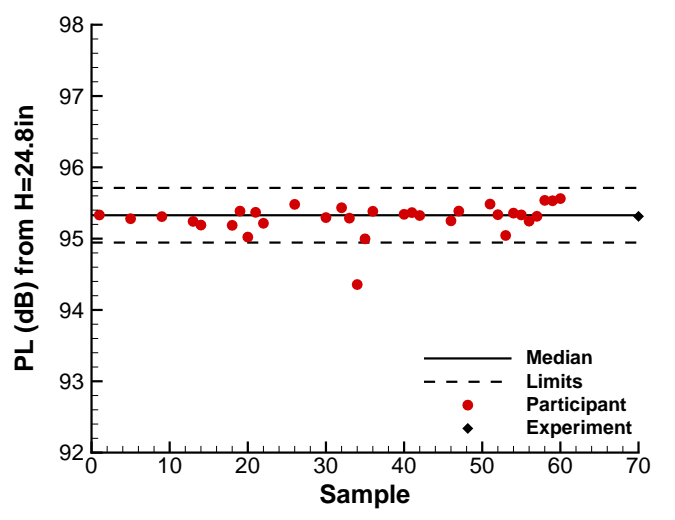

(b) Fine-grid submissions; $34 \mathrm{H}$ is below the limits.

Figure 21. DWB PL statistics propagated from $H=24.8$ in. and $\phi=0^{\circ}$. 


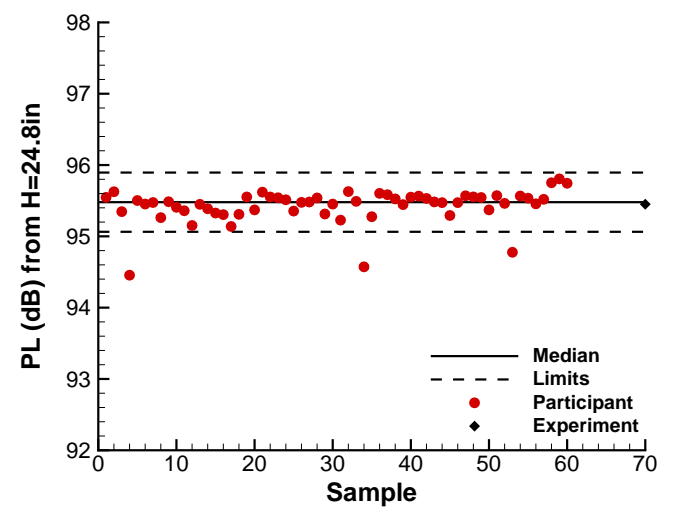

(a) All submissions; 4A, 34H, and 53N are below the limits.

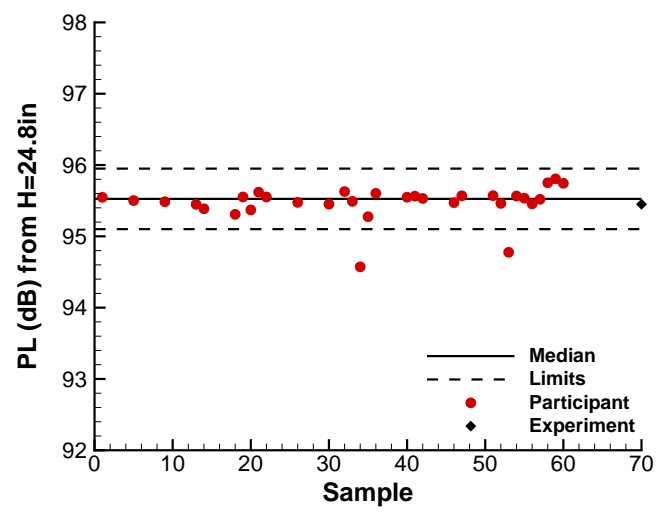

(b) Fine-grid submissions; $34 \mathrm{H}$ and $53 \mathrm{~N}$ are below the limits.

Figure 22. DWB PL statistics propagated from $H=24.8$ in. and $\phi=30^{\circ}$.

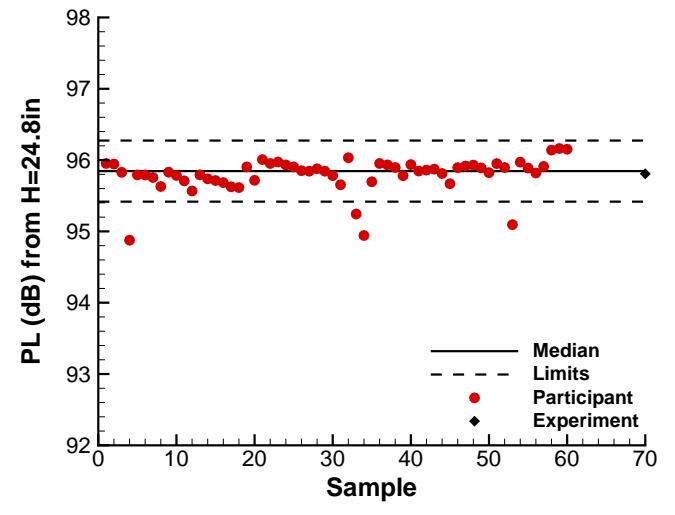

(a) All submissions; 4A, 33G, 34H, and 53N are below the limits.

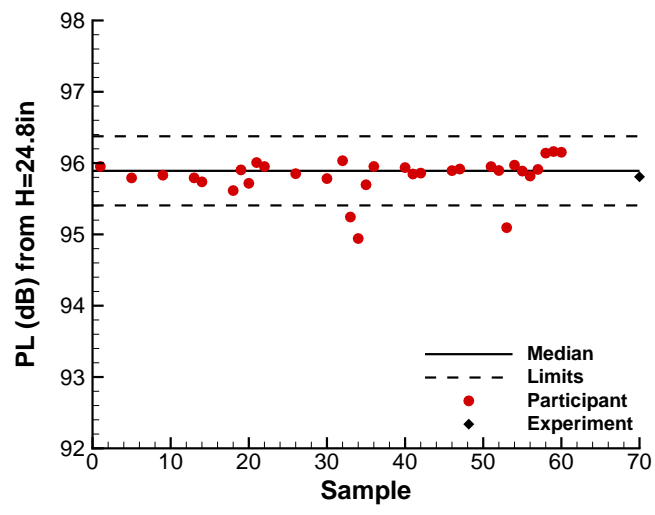

(b) Fine-grid submissions; 33G, 34H, and 53N are below the limits.

Figure 23. DWB PL statistics propagated from $H=24.8$ in. and $\phi=60^{\circ}$.

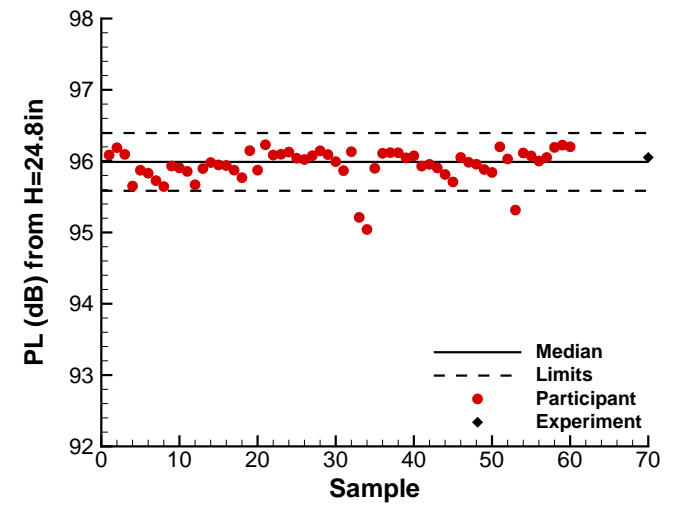

(a) All submissions; 33G, 34H, and 53N are below the limits.

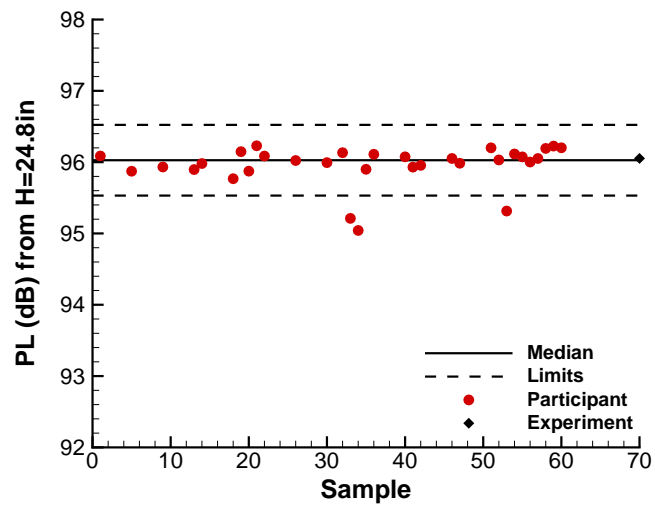

(b) Fine-grid submissions; 33G, 34H, and 53N are below the limits.

Figure 24. DWB PL statistics propagated from $H=24.8$ in. and $\phi=90^{\circ}$. 
centerline shock aligned Cartesian grid that has low resolution of the DWB base flow. The output adapted $34 \mathrm{H}$ submission is an outlier at and above $\phi=60^{\circ}$ and is the coarsest grid $(229,000$ control volumes) of the fine-grid submissions. Other participant $\mathrm{H}$ submissions based on a larger adapted and workshop provided mixed-element grids are not statistically significant at any $\phi$.

The PL of propagated submissions from three centerline locations is shown in Fig. 25(a) with PL of propagated mean wind tunnel measurements. The winged DWB configuration is insensitive to extraction distance over this range of extraction distances (3.1, 3.6, and 4.6 body lengths or 15.6, 18.3 and 23.4 half spans) at this non-lifting flow condition. The difference between the PL propagated from $H=31.8 \mathrm{in}$. and $H=24.8$ in. is shown in Fig. 25(b). The scatter of the DWB submissions is lower than the scatter of the SEEB-ALR submissions, Fig. 14(b). The ratio of extraction distances is also slightly less. The median of the PL differences is similar to the mean wind tunnel measurement PL difference. The coarse-grid 4A submission is above the limits and the $51 \mathrm{M}$ submission is slightly below the limits.

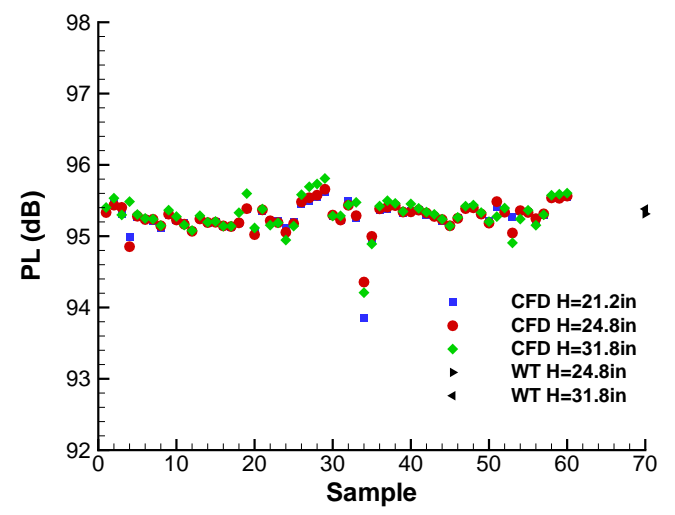

(a) PL propagated from three near-body distances.

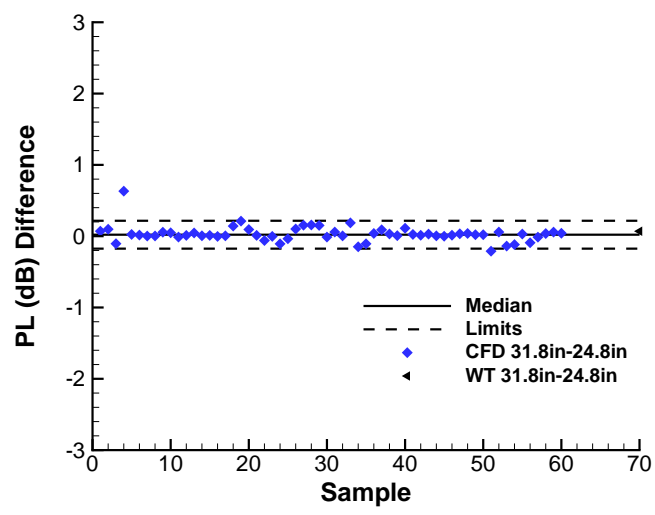

(b) Difference between $H=31.8$ in. and $H=24.8$ in. $4 \mathrm{~A}$ is above and $51 \mathrm{M}$ is below the limits.

Figure 25. DWB PL statistics propagated from three near-body distances on the centerline.

\section{VIII.E. DWB Outliers}

Submissions $33 \mathrm{G}, 34 \mathrm{H}$, and $53 \mathrm{~N}$ are identified as statistical fine-grid outliers in PL at multiple $\phi$. These submissions are not all outliers at every $\phi$-angle, but are shown at all $\phi$ to illustrate $\phi$-trends. The nearfield CFD pressure signatures are shown on the centerline (Fig. 26), $30^{\circ}$ off-track (Fig. 27), $60^{\circ}$ off-track (Fig. 28), and $90^{\circ}$ off-track (Fig. 29) with mean wind tunnel measurements. The wind tunnel mean shows the separation of the wing and base flow shocks and the steepening of the wing expansion with increasing off-track angle. Submission 33G has a unique shock in the middle of the main expansion that grows with $\phi$. Participant G indicated that the wing tip structured grid singularity may have adversely affected the CFD solver. The adapted-grid $34 \mathrm{H}$ submission predicts a lower pressure in the expansions than the wind tunnel mean, which has a constant expansion slope. Submission $53 \mathrm{~N}$ lacks distinguishing features that would indicate why it is an outlier.

One outlier for the difference in PL at two extraction distances remains after coarser uniformly refined grids are excluded. The near-field signature for the remaining outlier $51 \mathrm{M}$ is shown in Fig. 30. The slope of the main expansion is less steep for the $51 \mathrm{M}$ near-field centerline signature at $H=31.8 \mathrm{in}$. Also, the aft wing and base flow shocks have merged. This is the same behavior exhibited by most participants. It is unclear what subtle difference results in the reduction of $0.2 \mathrm{PL}(\mathrm{dB})$ from $H=24.8$ in. to $H=31.8$ in.

\section{LM1021 Analysis}

Some of the analysis steps from the SEEB-ALR and DWB are applied to the LM1021. The LM1021 has fewer submissions, so the previously used statistical methods are not be applied to the LM1021. The LM1021 submissions show a larger amount of variation in near-field and ground signatures than the SEEB-ALR or DWB, which may be due to the complexity of the model or the tailored nature of the low-boom signature. 


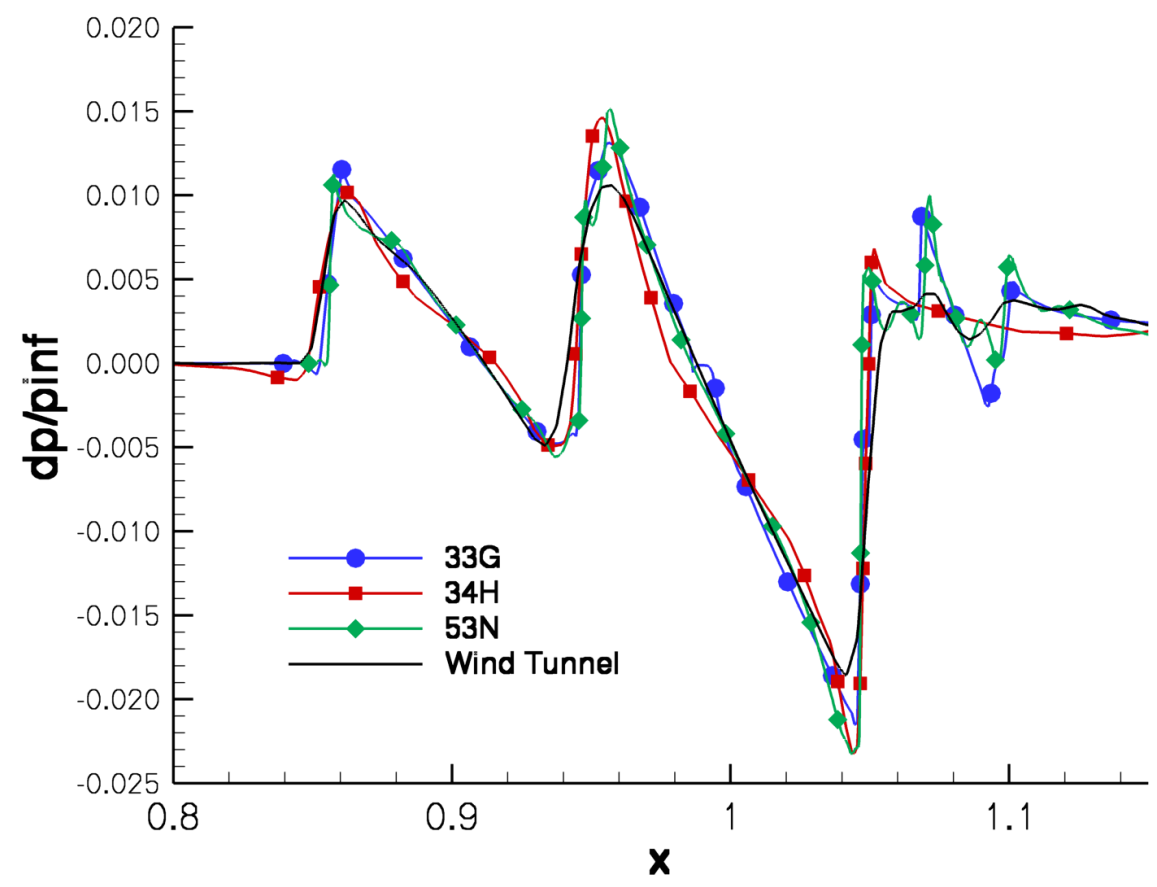

Figure 26. DWB fine-grid PL outliers: 33G, 34H, and 53N at $H=24.8$ in. and $\phi=00^{\circ}$.

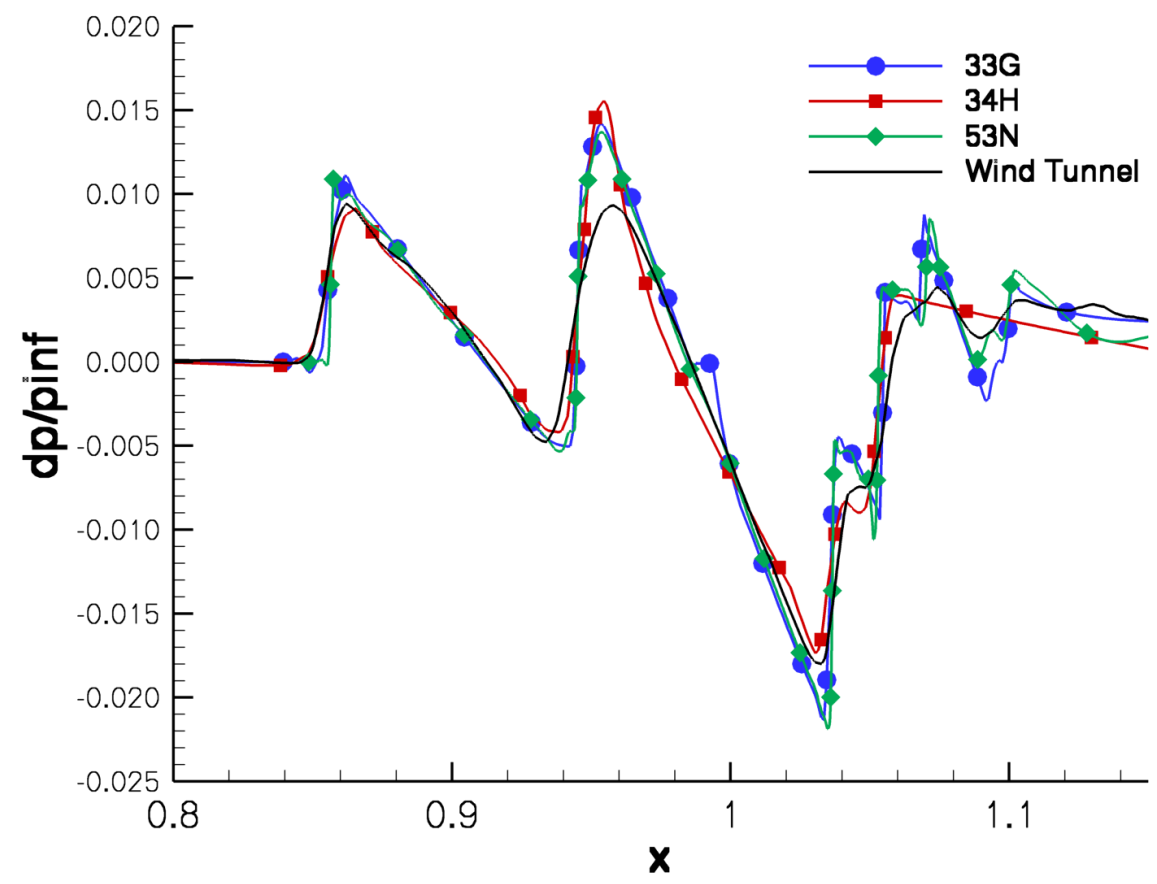

Figure 27. DWB fine-grid PL outliers: 33G, 34H, and 53N at $H=24.8$ in. and $\phi=30^{\circ}$. 


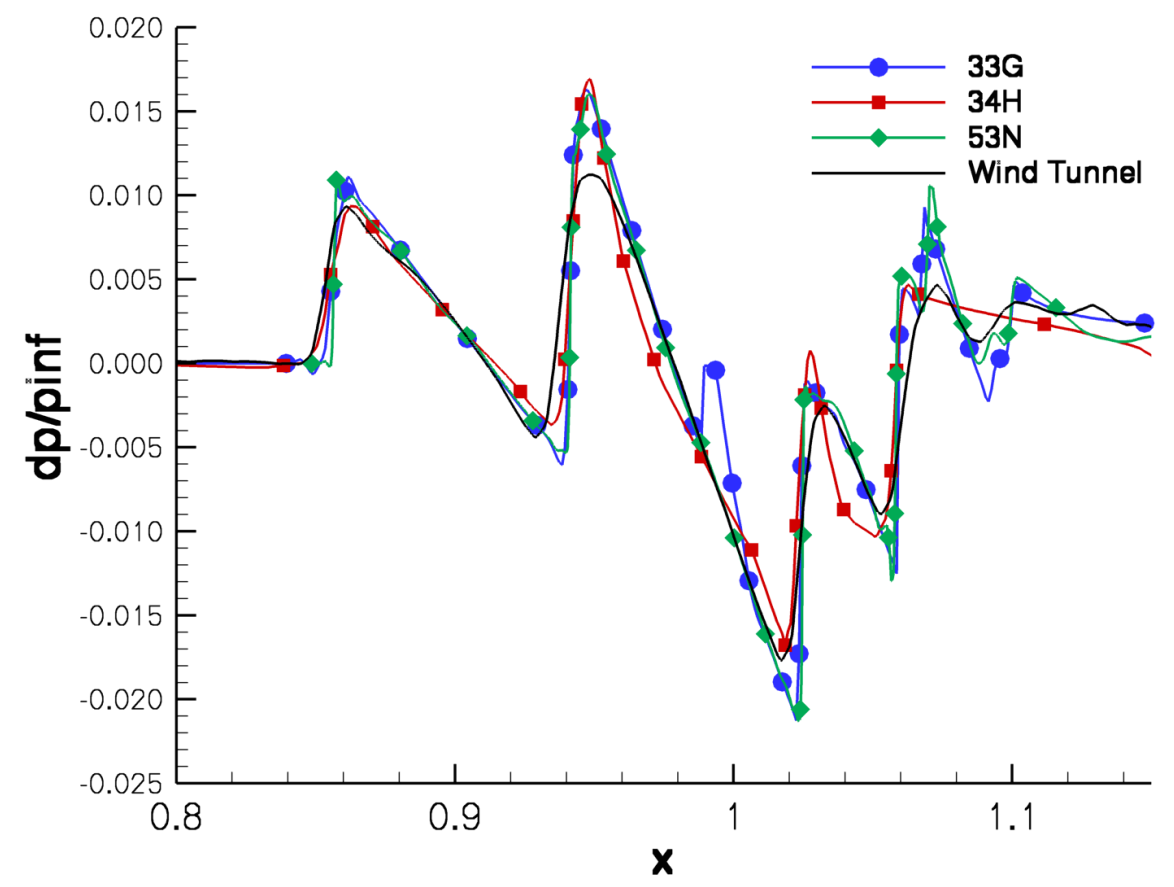

Figure 28. DWB fine-grid PL outliers: 33G, 34H, and 53N at $H=24.8$ in. and $\phi=60^{\circ}$.

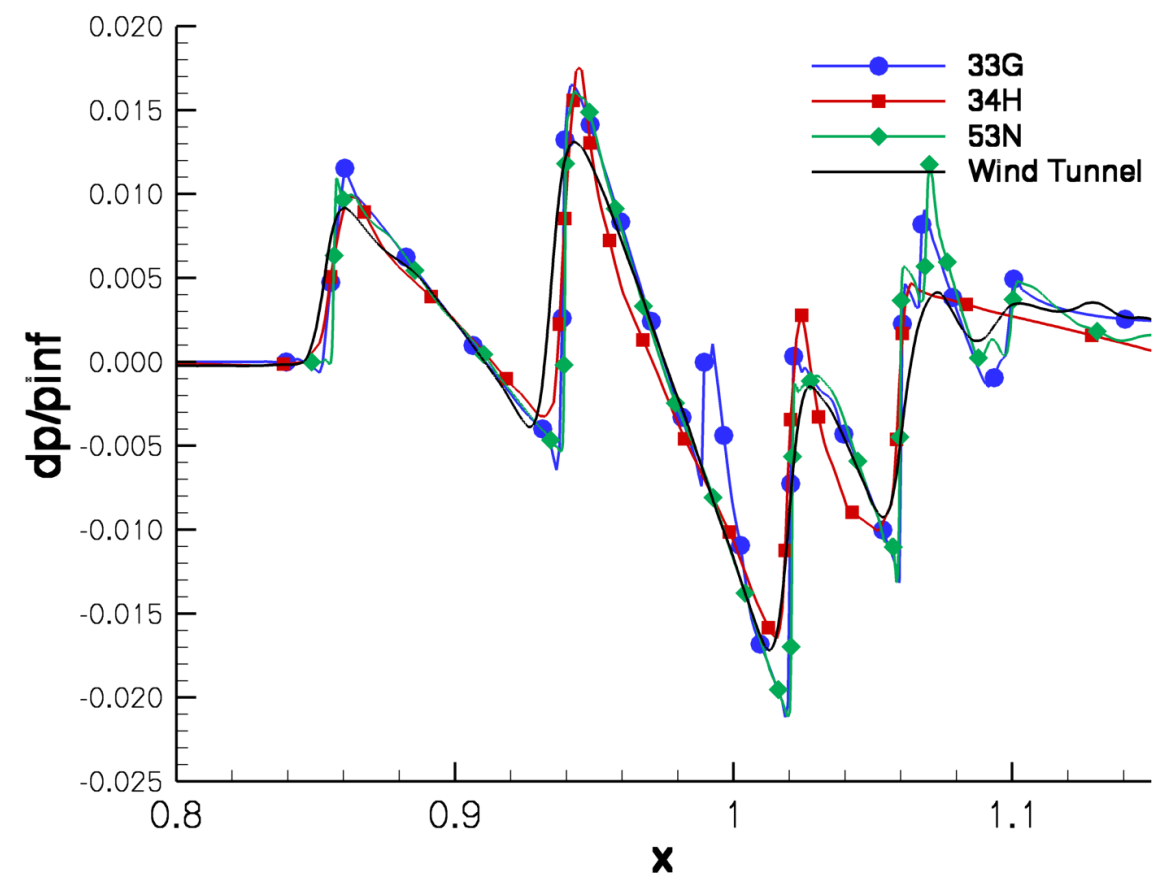

Figure 29. DWB fine-grid PL outliers: 33G, 34H, and 53N at $H=24.8$ in. and $\phi=90^{\circ}$. 


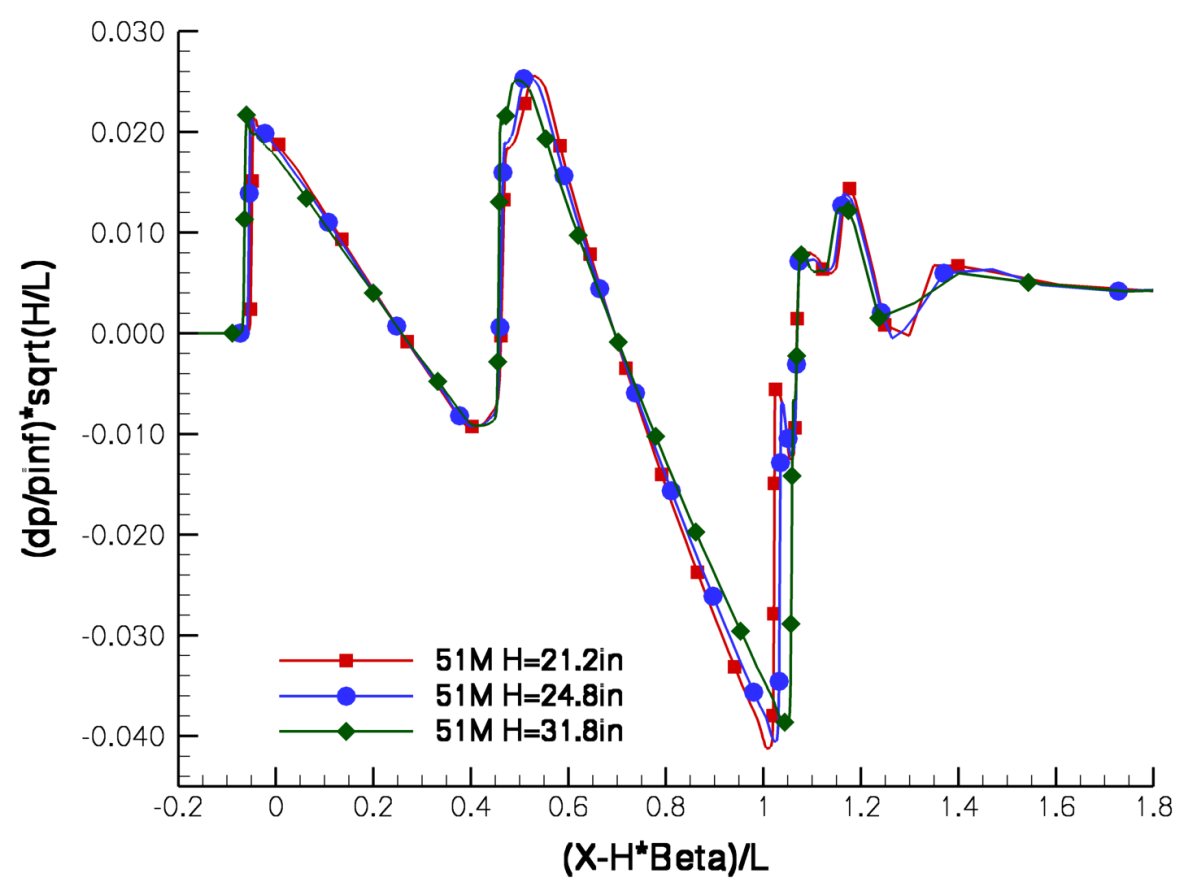

Figure 30. DWB fine-grid PL difference outlier: three near-body distances for scaled $51 \mathrm{M}$.

The variation near-field is also reflected in a large variation in PL and CSEL.

\section{IX.A. LM1021 Near-Body Signatures}

To introduce the LM1021, all of the submitted near-field signatures on the centerline at $H=31.8$ in. are shown in Fig. 31 as gray lines; CFD was requested at $2.10^{\circ}$ angle of attack. Mean wind tunnel measurements are shown from a number of test series in Fig. 31 for conditions near $H=31.8$, each with a different mean angle of attack. The $2.30^{\circ}$ angle of attack mean signature is computed with amplitude scaling, age scaling, reverse aging, auto reference clipping, and other processing features. Cliff et al. ${ }^{28}$ describes the method used to compute the other mean signatures. The wind tunnel mean pressure level at the forward portion of the fuselage $(X=[3.3,3.9])$, the forward portion of the wing $(X=[3.9,4.4])$, the wing shock position $(X=4.5)$, the nacelles $(X=[4.7,4.9])$, and the tail $(X>5)$ vary with angle of attack, wind tunnel test series, and spatial averaging technique. ${ }^{34,36}$ The angle of attack of the wind tunnel test is corrected with an upright and inverted measurement of lift. ${ }^{21}$ This process is complicated by flow angularity variation in the wind tunnel test section that results in $0.16^{\circ}$ peak-to-peak variation in angle of attack ${ }^{28}$ as the model is translated to perform spatial averaging. The $2.10^{\circ}$ angle of attack specified for workshop CFD computations is based on CFD performed before the workshop. ${ }^{6}$ These $2.10^{\circ}$ angle of attack pre-workshop simulations compared well to $2.30^{\circ}$ angle of attack wind tunnel measurements. There are also data reduction updates that indicated that the wind tunnel angle of attack may be lower than initially estimated.

All the submitted signatures from Table 8 are shown in Fig. 32 at $H=31.8$ in. on the centerline. There is a significant variation in pressure level over the entire signature and in the details of the main shock and expansion in the influence region of the nacelle $(X=[4.6-4.9])$. The variation in pressure level is largest at the tail of the configuration, especially the pressure recovery behind the model $(X>5.3)$. The differences in pressure level ahead of the wing $(X<4)$ may be due to differences in boundary layer displacement thickness. The submissions are grouped by physical model in subsequent figures.

The laminar and turbulent submissions are shown in Fig. 33. The Euler submissions are shown in Fig. 34. Referring to all submissions in Fig. 32, the laminar and turbulent submissions have a higher pressure for the forward portion of the signature than the Euler submissions. The predicted laminar and turbulent main shock location is ahead of the Euler main shock location. 


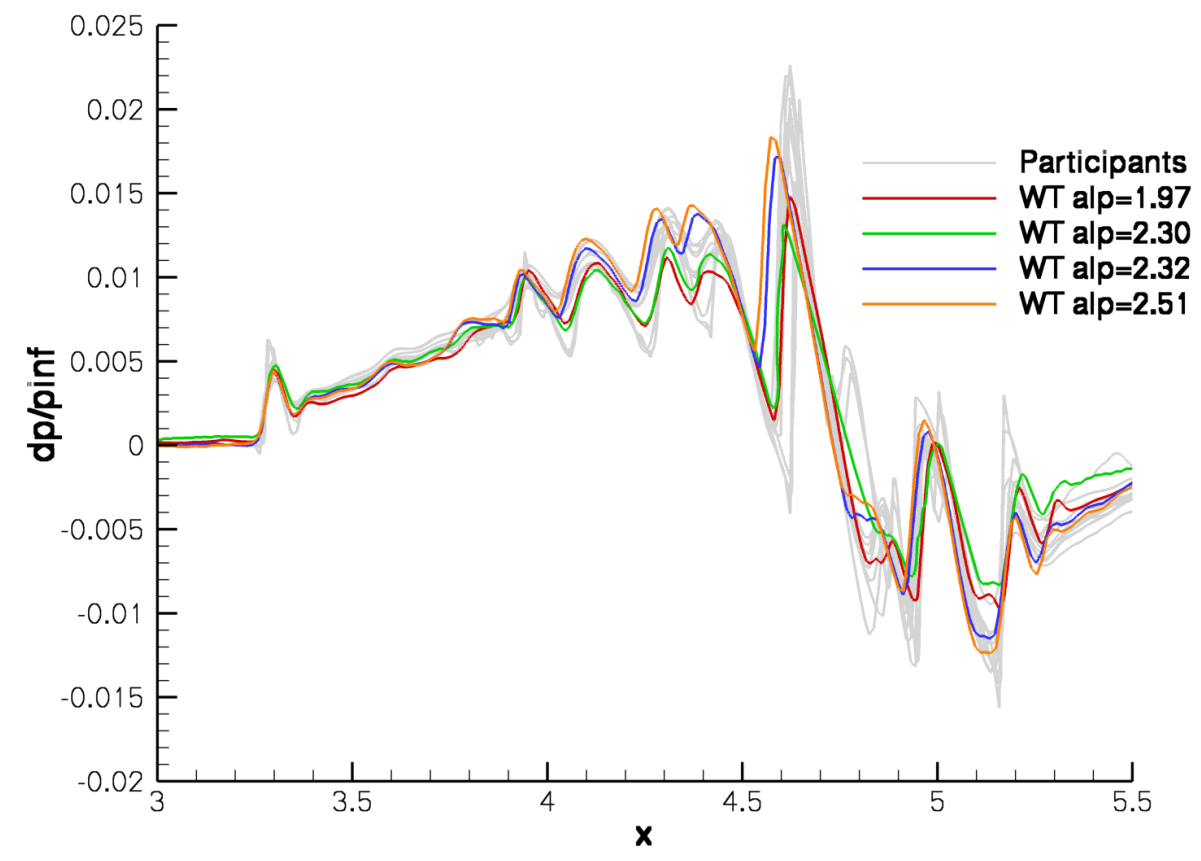

Figure 31. LM1021 near-field submissions and mean measurements at $H=31.8$ in., $\phi=0^{\circ}$.

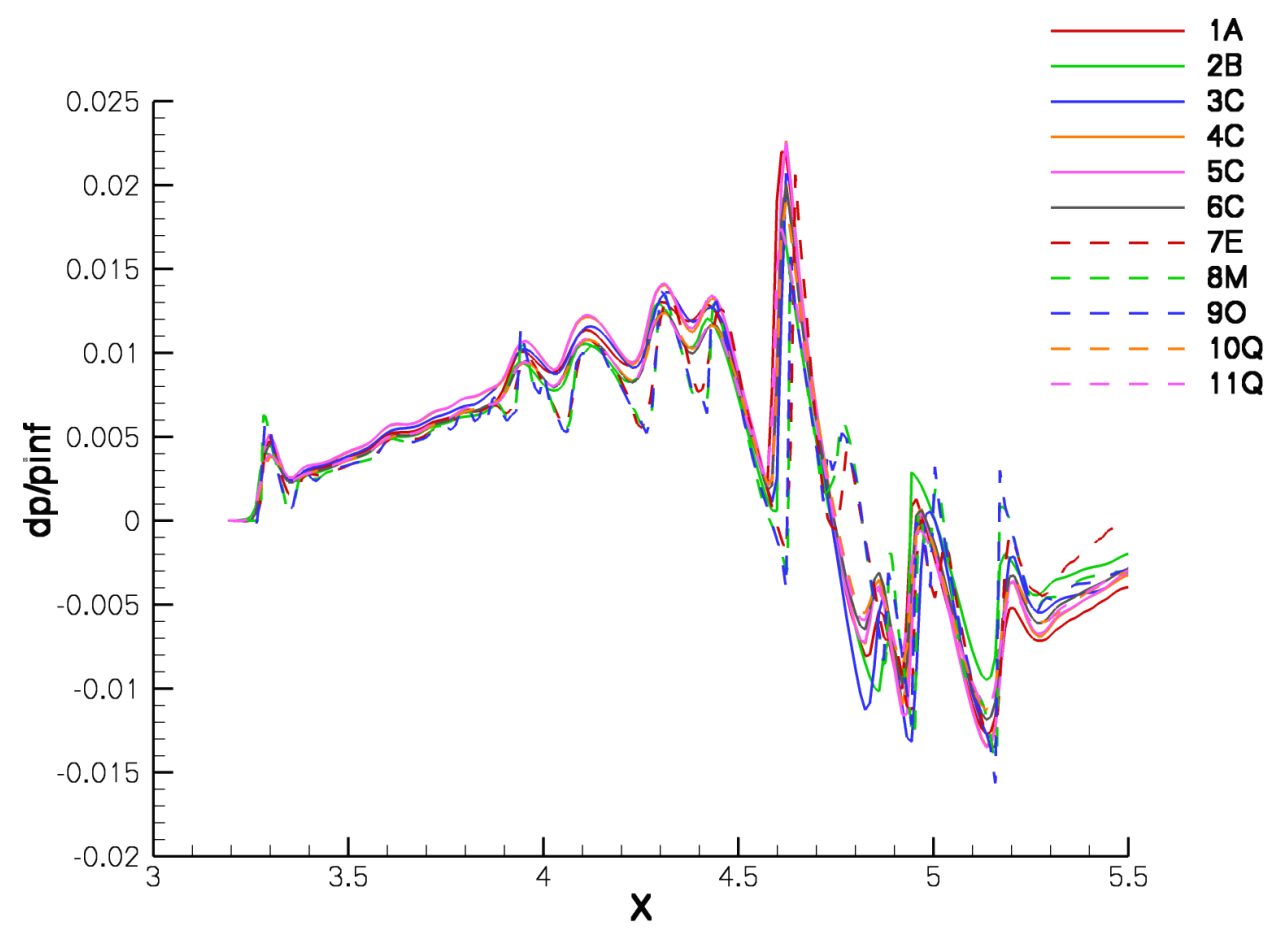

Figure 32. All LM1021 near-field submissions at $H=31.8$ in., $\phi=0^{\circ}$.

30 of 43 
There is little variation between the three Euler signatures (Fig. 34), except at the main shock, nacelle, and tail. There is a shock in the main expansion that is not seen in the laminar and turbulent submissions or wind tunnel mean measurements. This difference, unique to Euler calculations, may be due to the boundary-layer choking the wing-nacelle gap flow and preventing this shock from forming. ${ }^{12}$

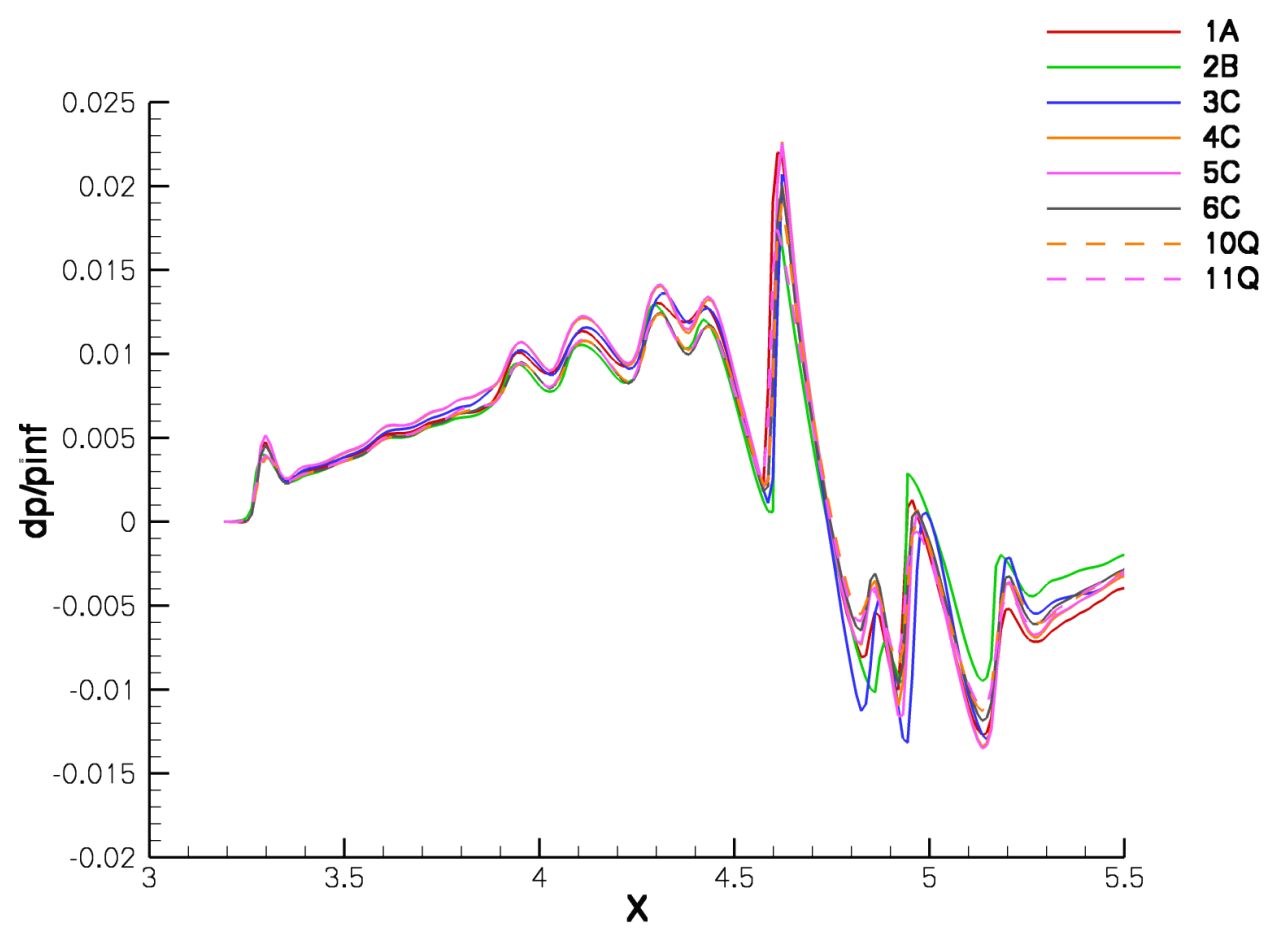

Figure 33. Laminar and turbulent LM1021 near-field submissions at $H=31.8$ in., $\phi=0^{\circ}$.

Participant $\mathrm{C}$ provided three calculations on the same grid, Fig. 35. These include a laminar calculation (3C) and turbulent calculations with the SA (4C) and SST (5C) turbulence models. The two turbulent simulations produce equivalent signatures. The laminar simulation has slightly lower pressure for the forward portion of the signature $X<4.4$ and the main expansion at $X=4.8$.

\section{IX.B. LM1021 Ground Signatures}

All the near-field signatures from Fig. 32 are propagated to the ground in Fig. 36. The signatures are propagated as provided by the participants without the application of a far-field correction ${ }^{30}$ that would be appropriate for signatures extracted this close to a vehicle with a spanwise distribution of acoustic disturbances. The signatures have been shifted in time to align the 0.2 PSF pressure rise to $100 \mathrm{~ms}$. The spread in forebody pressure seen in Fig. 32 results in a large spread in peak pressure in the first $50 \mathrm{~ms}$ of the ground signature. There is a large variation in the center shock location on the ground. The variation is smaller in the expansion after this shock. The location and strength of the final shock shows a large amount of variation.

The laminar and turbulent submissions shown in Fig. 33 are propagated to the ground in Fig. 37. The three Euler submissions shown in Fig. 34 are propagated to the ground in Fig. 38. The peak pressure of the laminar and turbulent submissions is higher and shows more variation than the three Euler submissions. The differences in the near-field main shock and nacelle region have manifested themselves as differences in the middle ground shock locations, where the middle Euler ground shock locations are all aft of the laminar and turbulent middle ground shock locations. With the exception of submission 2B, the laminar and turbulent tail shock locations are aft of the Euler tail shock locations. Submission 7E differs from the other two Euler signatures in shock locations and the shape of the final shock.

The participant C laminar near-field signature (3C) and turbulent near-field signatures with the SA (4C) and SST (5C) turbulence models are shown in Fig. 35 for the near-field and propagated to the ground in 


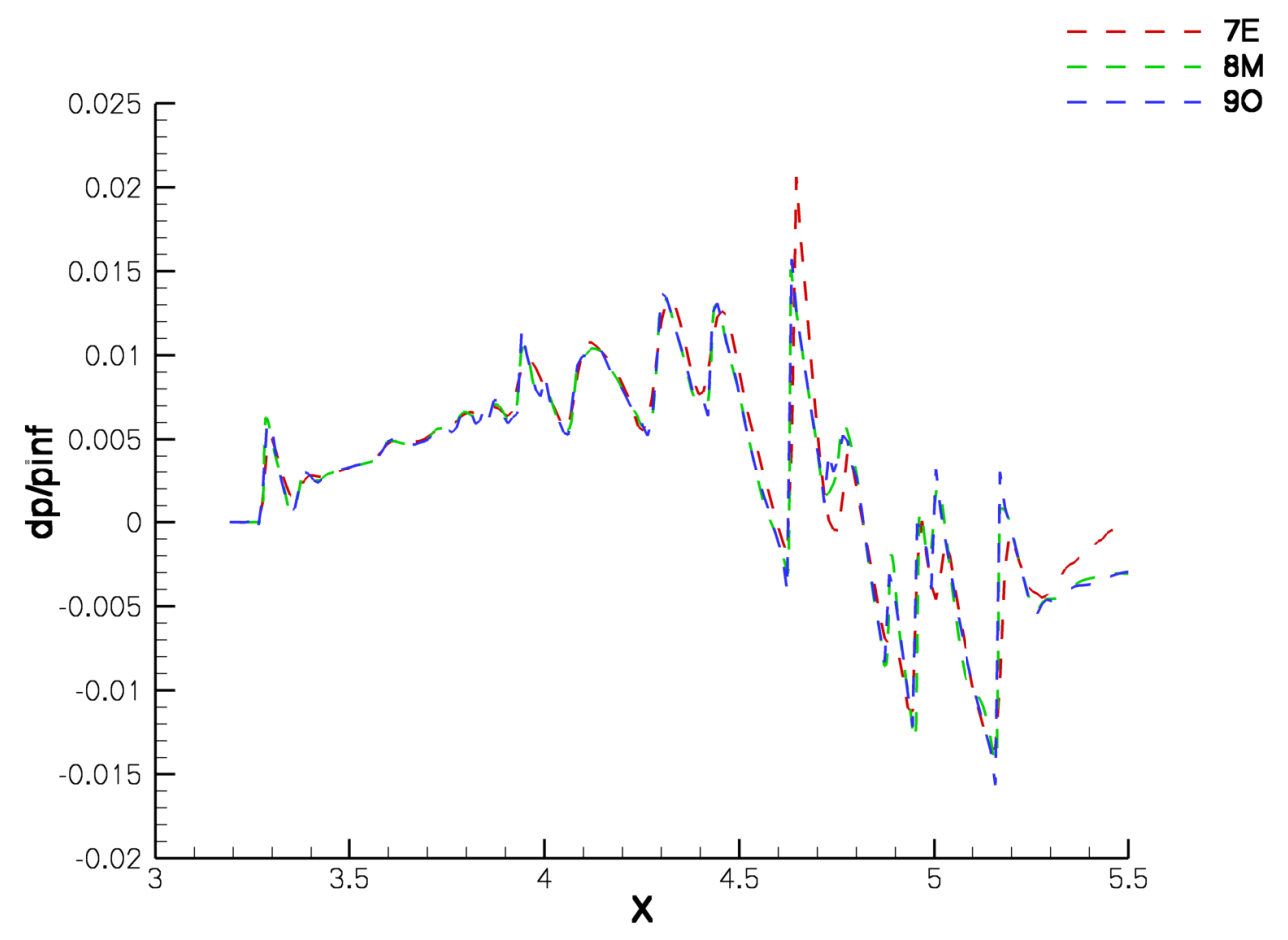

Figure 34. Euler LM1021 near-field submissions at $H=31.8$ in., $\phi=0^{\circ}$.

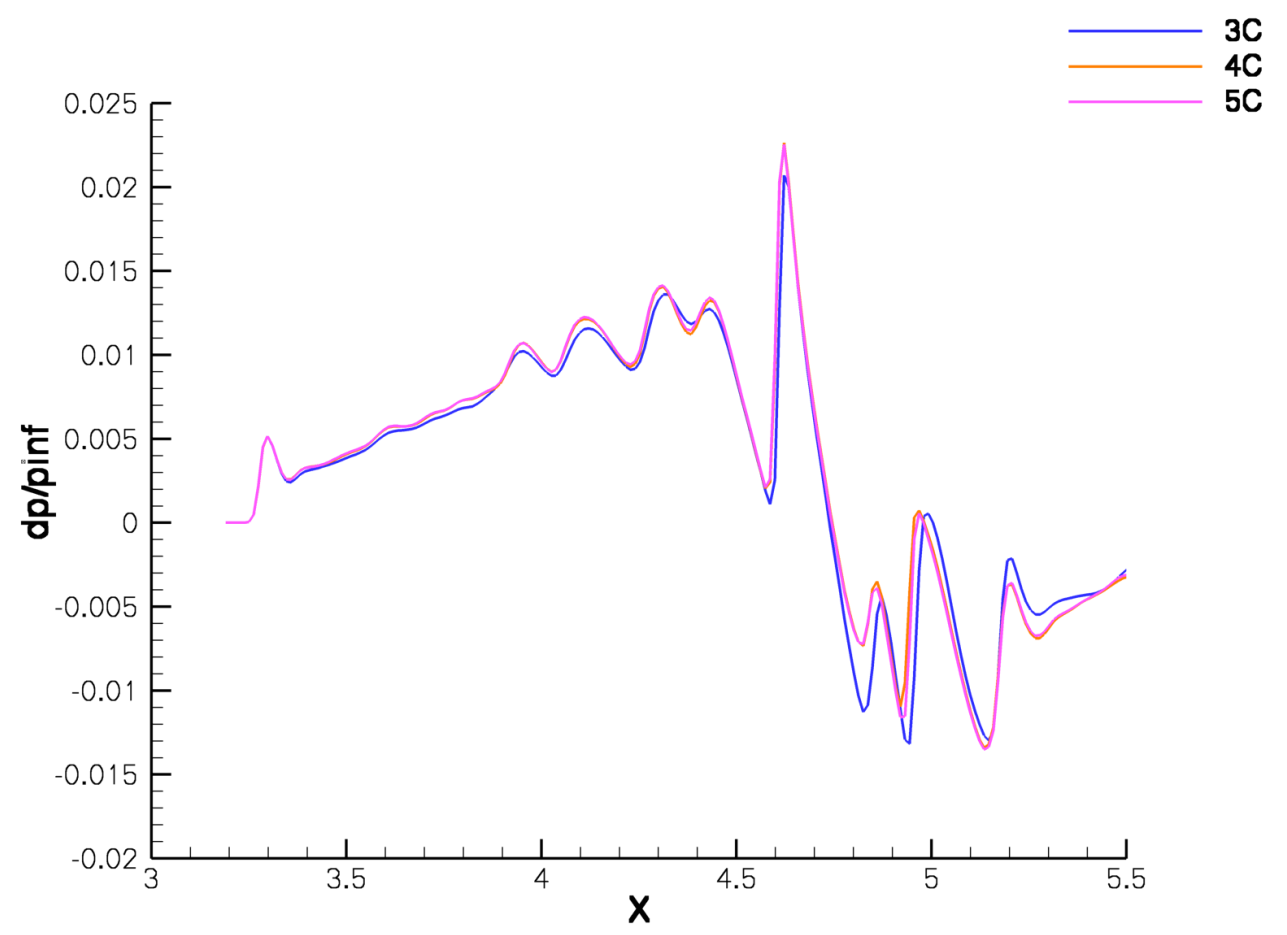

Figure 35. Laminar and turbulence model effects on LM1021 near-field signatures at $H=31.8$ in., $\phi=0^{\circ}$. 


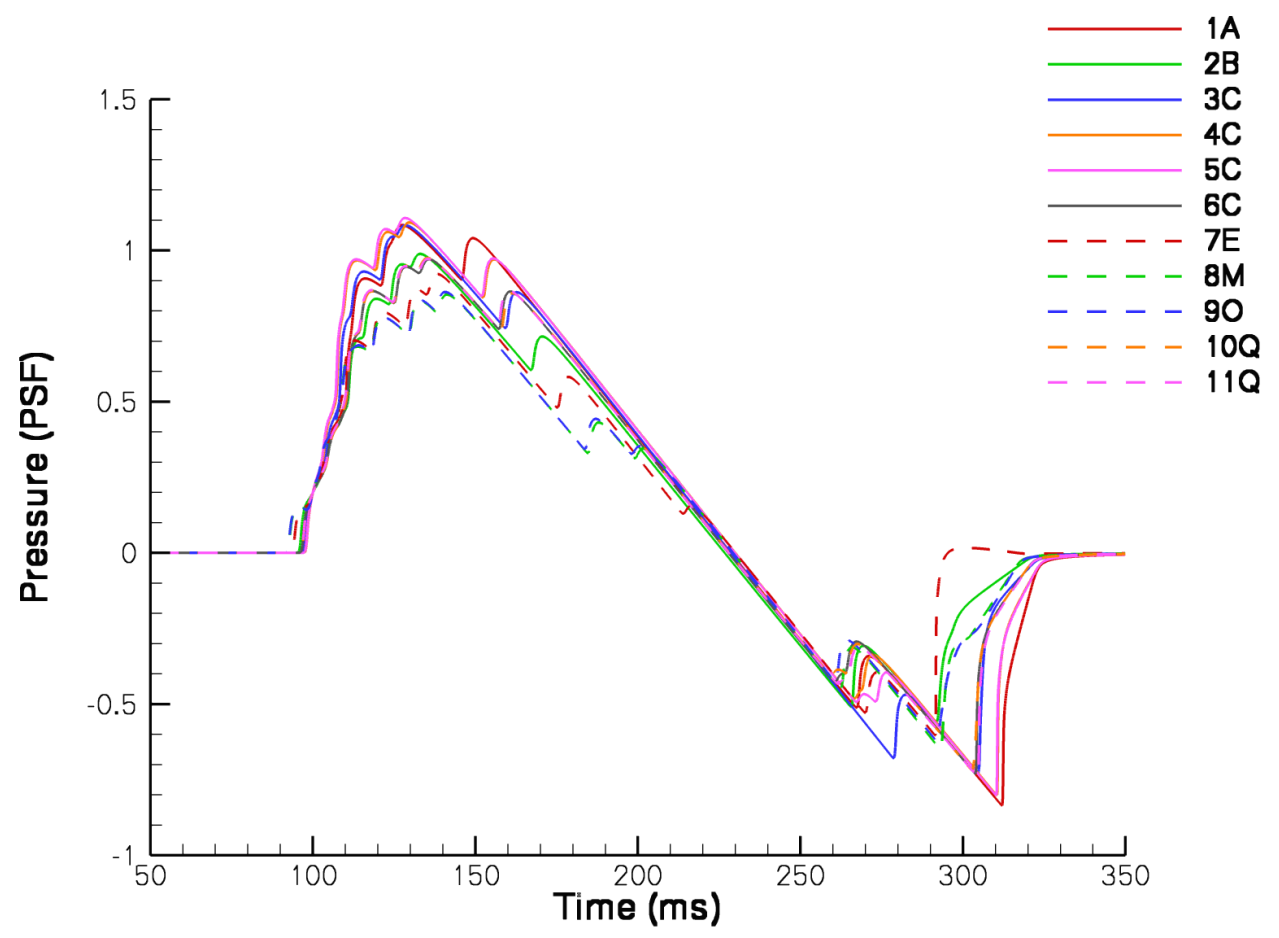

Figure 36. All LM1021 ground signatures from $H=31.8$ in., $\phi=0^{\circ}$.

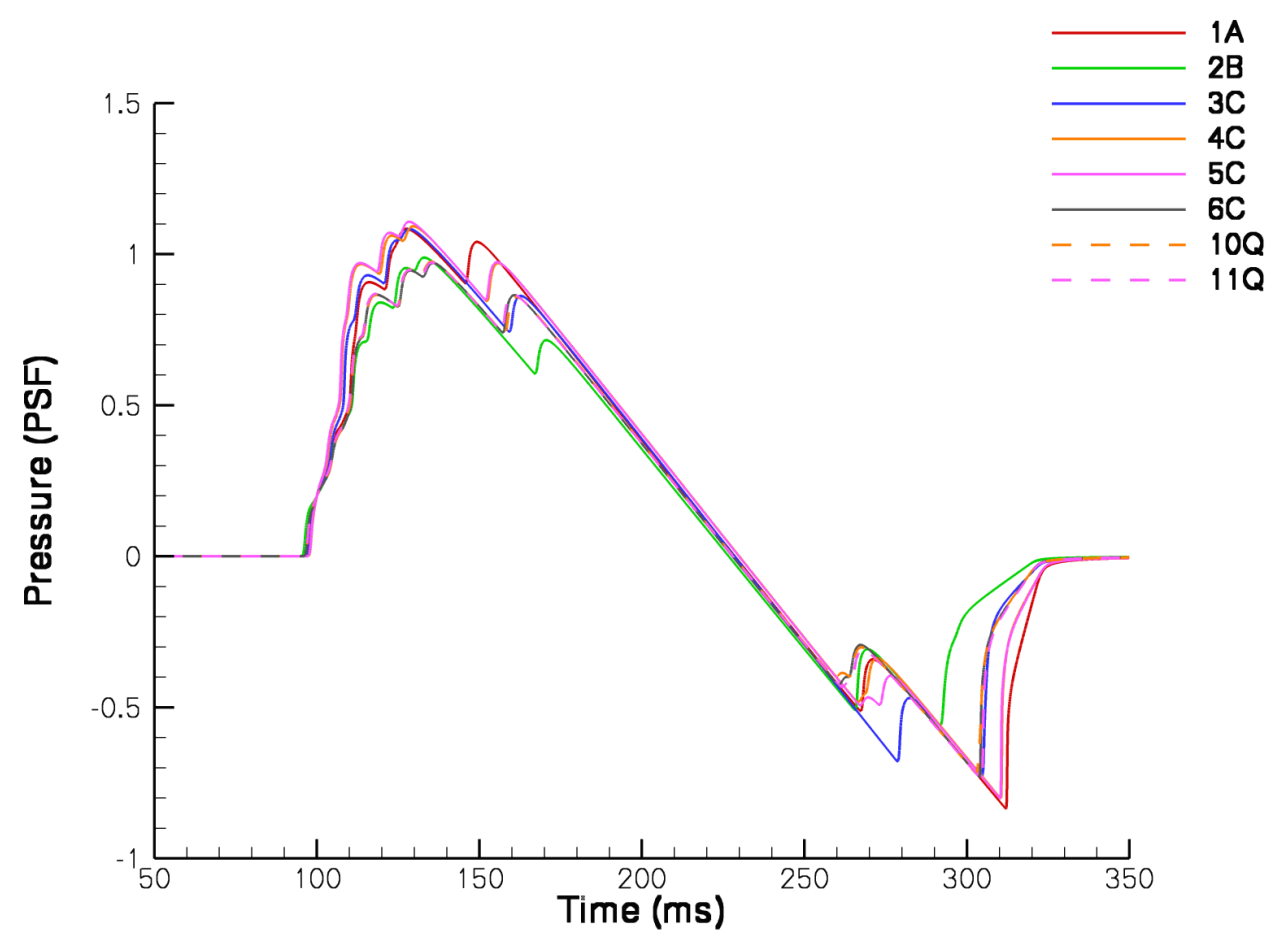

Figure 37. Laminar and turbulent LM1021 ground signatures from $H=31.8$ in., $\phi=0^{\circ}$. 


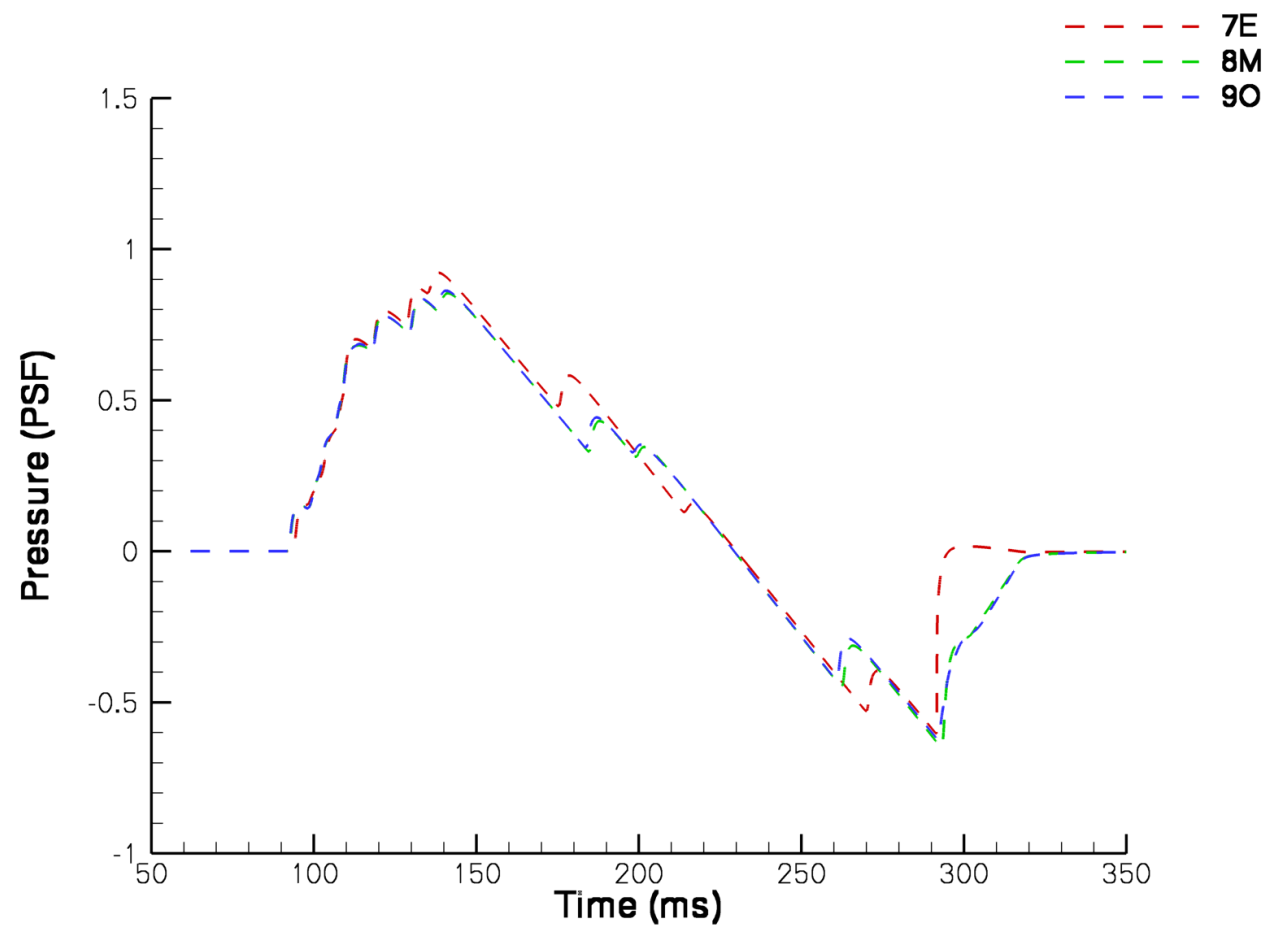

Figure 38. Euler LM1021 ground signatures from $H=31.8$ in., $\phi=0^{\circ}$.

Fig. 39. The two turbulence models only show differences in the nacelle influence region $(t=270 \mathrm{~ms})$. The laminar signature differs from the turbulent signatures primarily in shock locations. The laminar submission $3 \mathrm{C}$ shock located at $t=270 \mathrm{~ms}$ is in the most aft position of any submission.

\section{IX.C. LM1021 Loudness}

The PL and CSEL are computed from the propagated LM1021 ground signatures. In general terms, PL emphasizes the higher frequencies between 1,000 and 10,000 KHz and CSEL covers a lower and wider frequency range between $100 \mathrm{~Hz}$ and a $5,000 \mathrm{KHz}$. These measures are shown as a function of near-field extraction distance on the centerline in Fig. 40. These measures are also presented in Fig. 41 as a function of off-track angle $\phi$ for the signatures extracted at $R /(b / 2)=7.9$. In contrast to the DWB, each off-track angle for the LM1021 is propagated obliquely through the atmosphere creating a "boom carpet."

The signatures are propagated as provided by the participants without the application of a far-field correction $^{30}$ that would be appropriate for signatures extracted this close to to the vehicle. This correction is omitted because it eliminates some of the differences between submissions that this summary intends to quantify. The combination of no far-field corrections, inclusion of blade model support, and simulating the wind tunnel Reynolds number results in a PL that is louder than a full-scale free-flight model, see section V.C for details.

A series of uniformly refined grids were not provided for the LM1021 configuration. The lack of signatures computed on a series of uniformly refined grids combined with the small number of submissions hinders the extraction of patterns from the LM1021 loudness, but the following observations are offered. The viscous methods approach 91-94 dB PL and 99-97 dB CSEL at far $R$ with the exception of 2B, 10Q, and 11Q. These submissions have a lower pressure than other viscous submissions in the front portion of the nearfield signature (Fig. 33) that results in a lower peak pressure (Fig. 37). Submission 2B has a high pressure level in the aft part of the near-field signature that results in a forward tail shock location (near the tail shock location of the Euler methods). The Euler methods are near $86 \mathrm{~dB}$ PL and $95 \mathrm{~dB}$ CSEL with the exception of 7E. Submission 7E has a unique ground tail shock position and shape, Fig. 36 and Fig. 38. Submission 1A has the greatest increase in PL with $\mathrm{R}$ and submission 10Q has a unique drop in CSEL level with increasing $\mathrm{R}$. With the exception of $10 \mathrm{Q}$, all the participants show less $R$ variation in CSEL than 


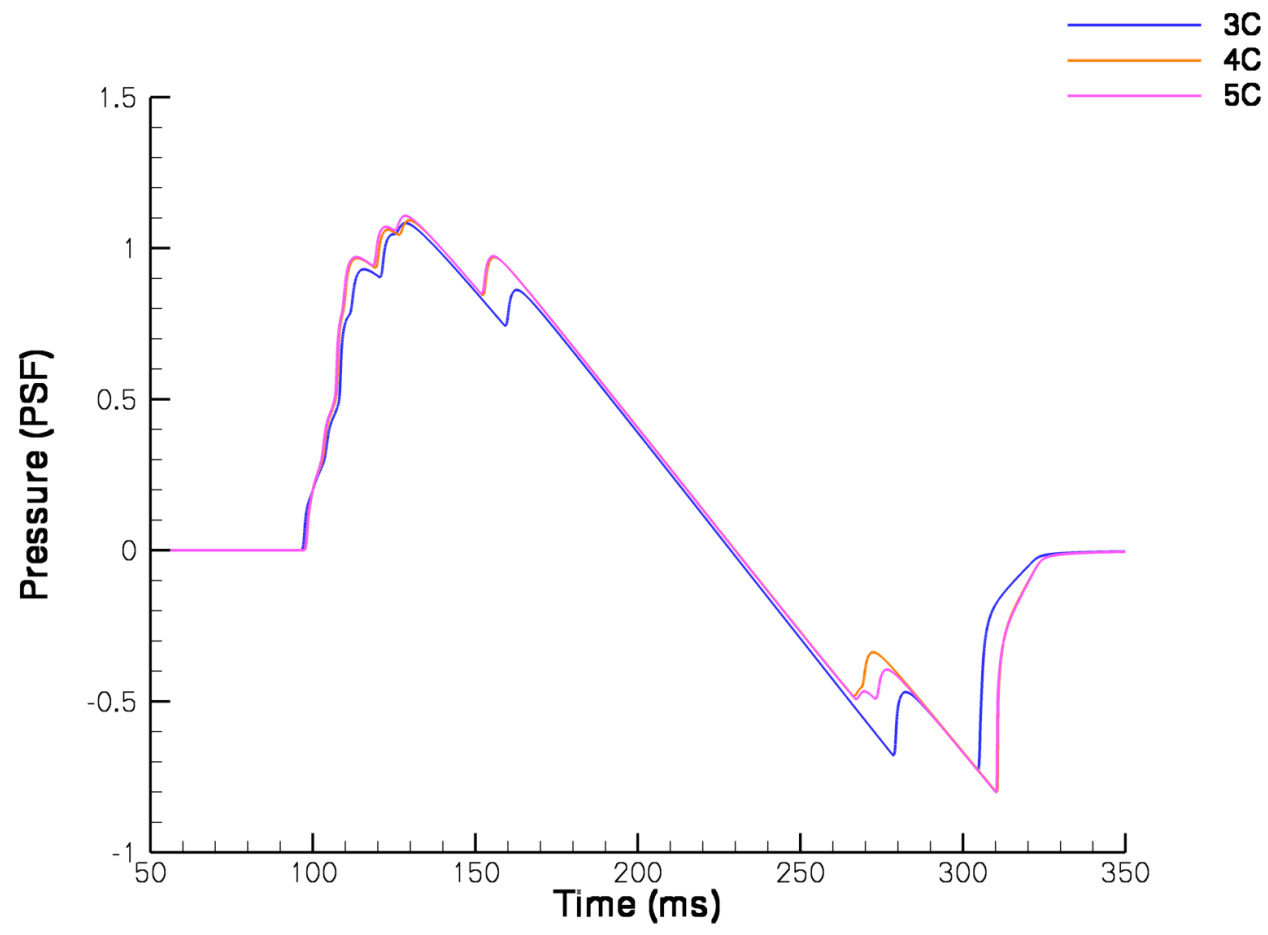

Figure 39. Laminar and turbulence model effects on LM1021 ground signatures propagated from $H=31.8$ in., $\phi=0^{\circ}$.

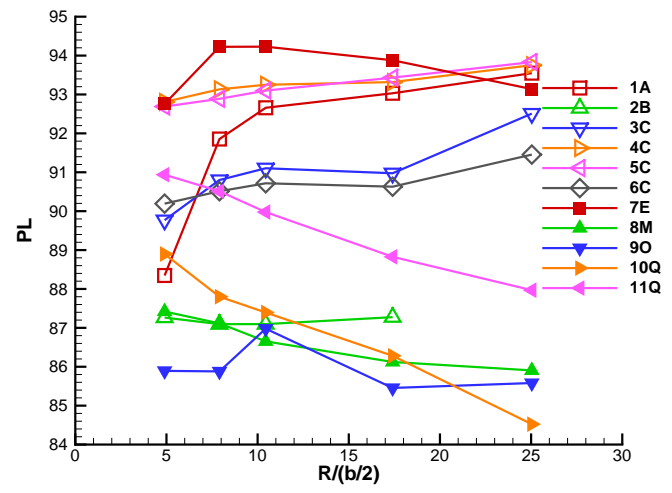

(a) PL.

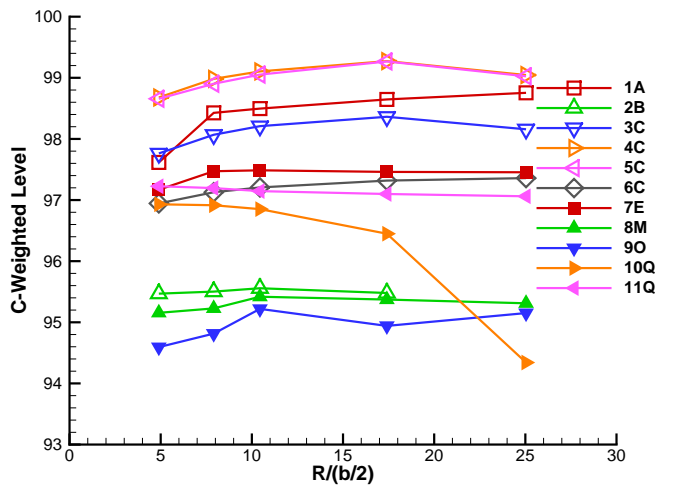

(b) CSEL.

Figure 40. LM1021 loudness variation with near-body distance at $\phi=0^{\circ}$. 


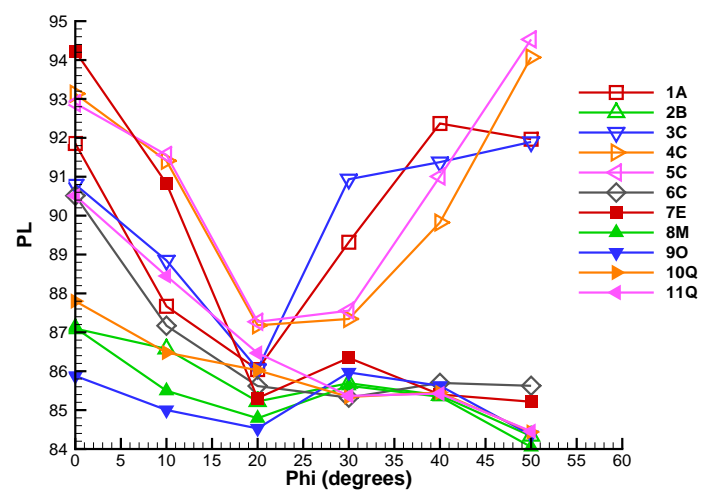

(a) PL.

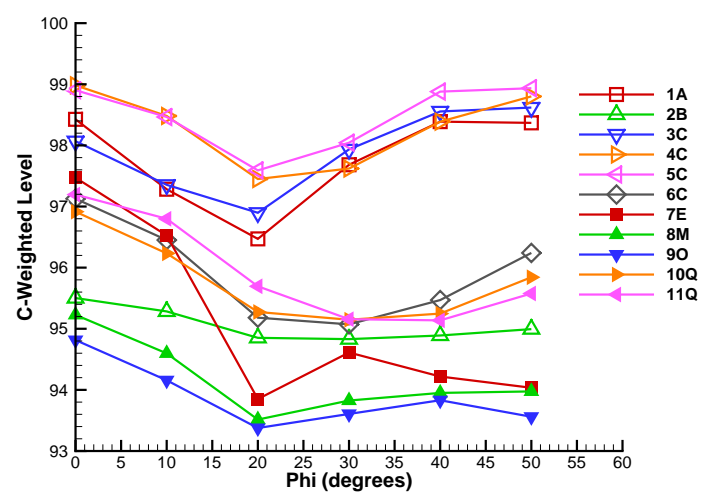

(b) CSEL.

Figure 41. LM1021 loudness variation with off-track angle at $H=31.8$ in

PL. CSEL emphasizes more of the low frequency content of the ground signature than PL, which indicates the propagated ground signatures have more high frequency changes with extraction distance $R$ than low frequency changes. Sensitivity analysis of LM1021 PL and CSEL measures indicates that both of these measures are sensitive to angle of attack (lift). ${ }^{43}$

The trends with off-track angle at $R /(b / 2)=7.9$ are shown in Fig. 41. The PL and CSEL of all submissions decrease between $0^{\circ}$ and $20^{\circ}$ off track, where the variation in PL is the least. The trend with increasing off-track angle is less consistent above $20^{\circ}$ off track with one group growing louder and the other staying nearly constant, The same louder and quieter groups are present in CSEL. The PL differences between $10 \mathrm{Q}$ and $11 \mathrm{Q}$, seen on the centerline, trend toward zero at $50^{\circ}$ off track. Submission $6 \mathrm{C}$ with a custom grid is quieter than other participant $\mathrm{C}$ submissions on the committee provided grid. A possible explanation of $6 \mathrm{C}$ off-track quieting could be differences in the off-track grid resolution between the committee provided and $6 \mathrm{C}$ grid.

The two submissions from participant Q are the same CFD method applied to the committee provided grid (10Q) and a custom grid (11Q). These two scaled and windowed near-field signatures are nearly identical except for aft pressure peaks, Fig. 42. These small differences result in very fine changes to the ground signatures, Fig. 43. However, there is approximately a $3 \mathrm{~dB}$ PL offset over a range of $R /(b / 2)$ extraction locations, Fig. 40. Examining these ground signatures in the frequency domain (Fig. 44) shows that they are identical below $100 \mathrm{~Hz}$, but 11Q has more energy above $100 \mathrm{~Hz}$. Applying an A-weighted digital filter to the ground signatures isolated the differences to the sharpness of the tail shock. ${ }^{44}$ The A-weighted loudness shows the same $3 \mathrm{~dB}$ difference between the Q submissions as PL, but CSEL only shows a $0.1 \mathrm{~dB}$ difference between $10 \mathrm{Q}$ and $11 \mathrm{Q}$ at $R /(b / 2)=7.9$. This is due to CSEL including more lower frequency energy than PL. These lower frequencies are identical between the two ground signatures, Fig. 44. The subtle differences between 10Q and 11Q may be useful as an evaluation tool for propagation and loudness measures.

\section{IX.D. LM1021 Far-Field Correction}

The spanwise distribution of disturbance sources cause near-field interference because these disturbances reach a near-field extraction location after traveling different distances. The CFD extraction location should be many times the semi-span of the model to minimize this near-field interference effect. When signatures are extracted sufficiently far from the model, a far-field propagation technique (sBOOM) is usable without correction because the acoustic sources appear to come from a single line.

CFD data is requested for an complete cylinder around the configuration to enable a far-field correction methodology that accounts for a spanwise distribution of acoustic disturbances. ${ }^{30}$ The average difference in PL of signatures propagated with and without this correction is shown in Fig. 45(a) as a function of $R /(b / 2)$. The impulse of this near-field signature correction is shown in Fig. 45(b). Both measures of the far-field correction decay with $R$. Far-field correction impulse shows a very steady hyperbolic convergence. The convergence of PL is less regular because the far-field correction changes the shape of the signature at different $R /(b / 2)$ locations. This shape change may result in an increase, decrease, or no change to PL. 


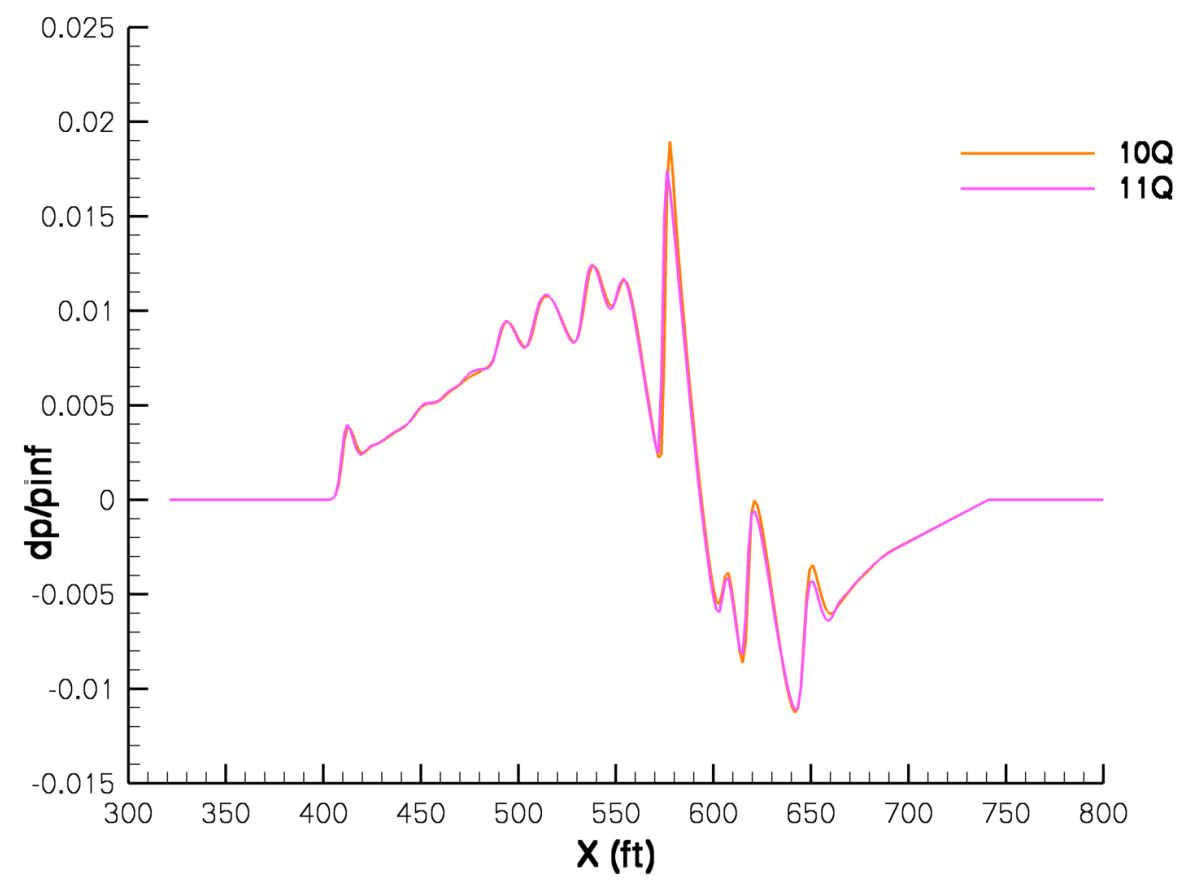

Figure 42. LM1021 full-scale near-field signatures for participant $\mathbf{Q}$ at $H=31.8$ in., $\phi=0^{\circ}$.

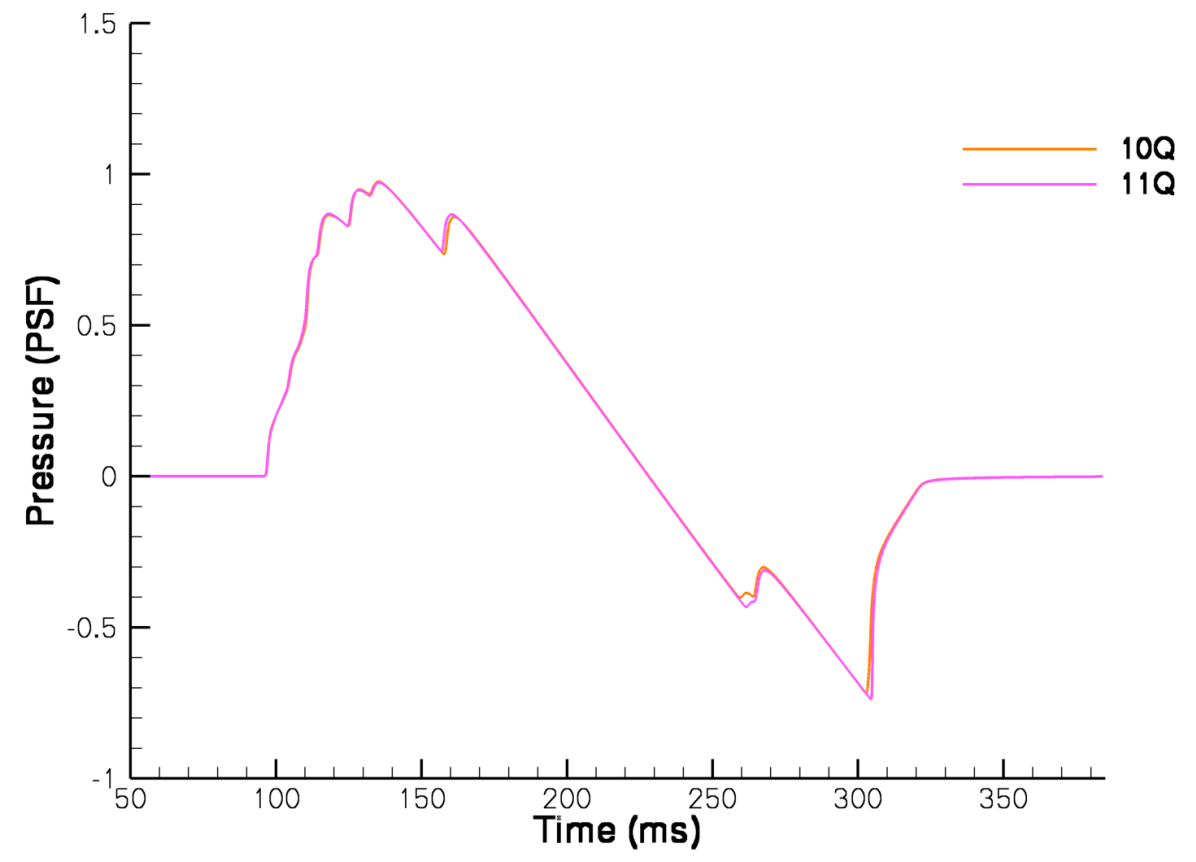

Figure 43. LM1021 ground signatures for participant $\mathbf{Q}$ from $H=31.8$ in., $\phi=0^{\circ}$. 


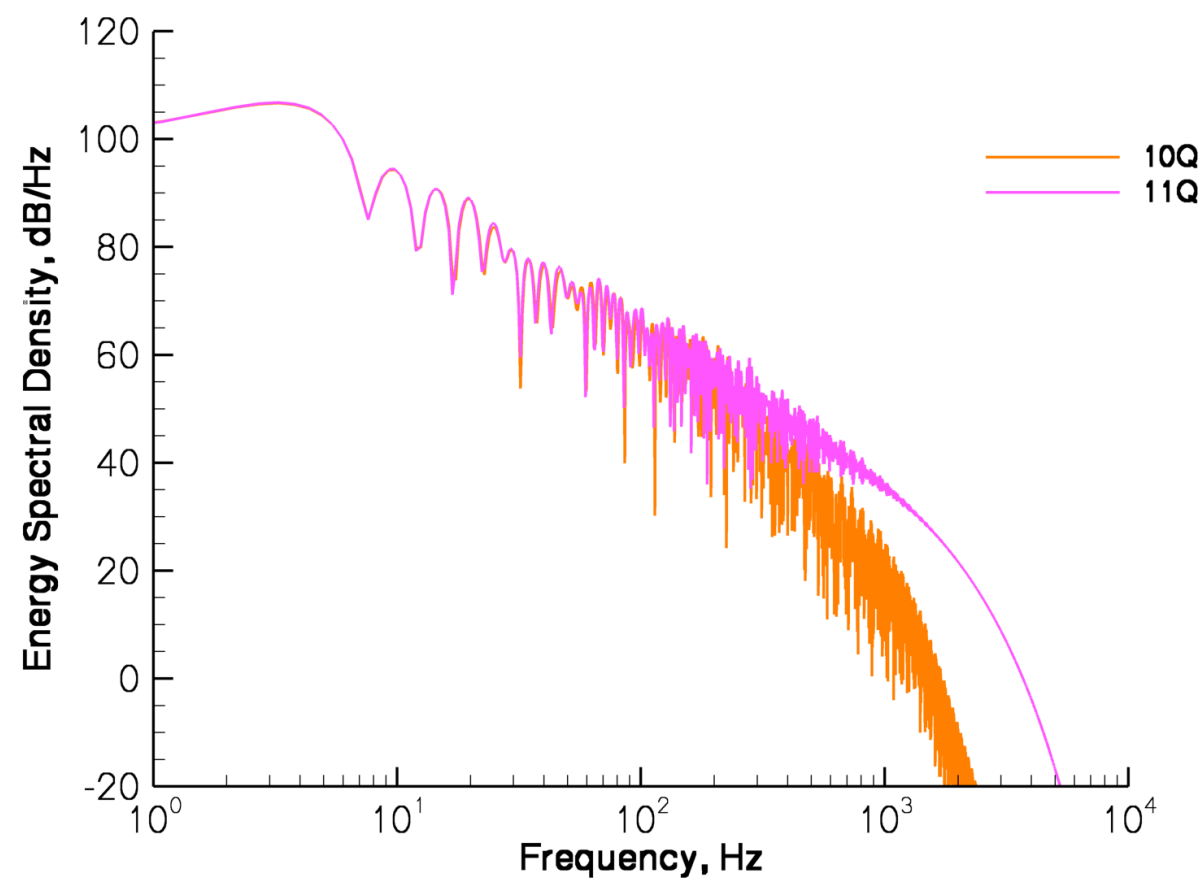

Figure 44. LM1021 ground signature spectra for participant $\mathbf{Q}$ from $H=31.8$ in., $\phi=0^{\circ}$.

Intermediate extraction locations may be useful for quantifying design changes, but a large $R /(2 / b)>25$ extraction distance is required for far-field propagation methods to obtain loudness measures within a single $\mathrm{dB}$ without a far-field correction.

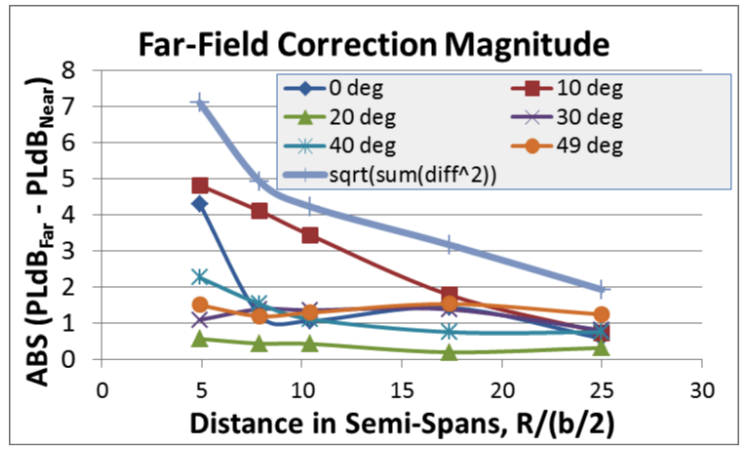

(a) PL.

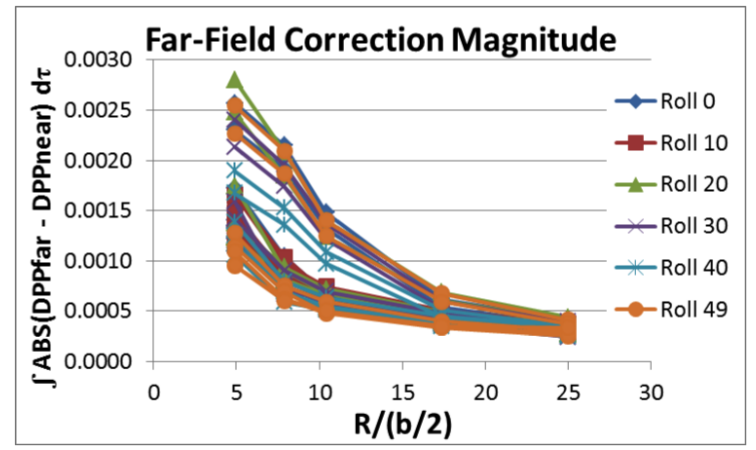

(b) Impulse.

Figure 45. LM1021 far-field correction decay with near-body distance.

\section{IX.E. LM1021 Equivalent Area}

Integrating the CFD signature into an equivalent area by the method of Whitham ${ }^{45}$ as extended by Walk$\operatorname{den}^{46}$ quantifies the overall integrated strength of each signature. Equivalent area is computed for each participant that provided an entire half cylinder of extracted pressures, Fig. 46. The equivalent area increases linearly with total lift (boundary layer displacement is a small additional contribution), so the CFD differences correspond to a $\pm 15 \%$ variation in lift for these submissions. Submissions $7 \mathrm{E}$ and $9 \mathrm{O}$ are Euler, submission $3 \mathrm{C}$ is laminar, and the other submissions are viscous. These two Euler submissions have a lower equivalent area than the laminar or turbulent submissions. The average equivalent area of the viscous solutions is approximately 80 square feet more than the average Euler submission. The $\pm 6 \%$ variation between 
the viscous submissions may be due to differences in lift or boundary layer state.

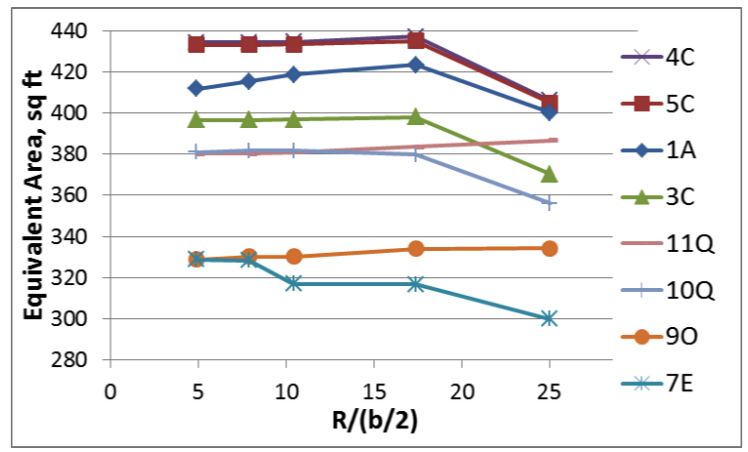

Figure 46. LM1021 equivalent area.

The equivalent area calculation includes scaling for the square root of $R$ dependency of pressure. Each submission should have constant area value when calculated from any of the 5 provided $R /(b / 2)$ distances. These solutions show mostly constant areas except for an abrupt reduction between 17.4 and $25 R /(b / 2)$ for all but two of the solutions (11Q and 9O). Submissions that exhibit a change in equivalent area may indicate that grid resolution is insufficient for accurate results at theses larger distances. The $7 \mathrm{E}$ and $9 \mathrm{O}$ Euler submissions have the same equivalent areas at $R /(b / 2)<=8$, but very different PL and CSEL in Fig. 40.

\section{Recommendations}

The iterative convergence of supersonic flows is complicated by the use of reconstruction limiters. ${ }^{47}$ However difficult, an iterative convergence criteria should be established or iterative convergence histories should be requested from participants. Insufficient iterative convergence was detected in the workshop uniform grid refinement studies of some participants, which encouraged the participants further converge the CFD for this summary. The convergence of forces is often monitored as a surrogate, but participant J showed in their workshop presentation that near-field signatures are still converging after the forces have reached steady state. This indicates that monitoring the iterative convergence of the solution at the extraction location may be a useful and possibly necessary tool.

Length units should be standardized for future workshops. The SEEB-ALR and LM1021 geometry and grids were provided in inches, but the DWB grid and geometry was provided in meters. The SEEB-ALR signatures were required in inches, the DWB signatures were required in meters, and the LM1021 signatures were required in feet. The use of different systems of units unduly confused participants and complicated the analysis of submissions.

Having a common grid system that the most participants could run was critical to understanding the results provided on custom and adapted grids. These committee provided grids identified problems with iterative convergence, post processing, and grid adaptation that would have been impossible to isolate if the participants had only used custom grids. Approximately half of the participants ran multiple grids in the workshop required uniform grid refinement series. These uniformly refined signatures were invaluable for understanding the convergence of PL and $V$ measures. A continued effort should be made to ensure that as many participants as possible can run multiple grids in a uniformly refined grid series with as many CFD methods as possible. LM1021 submissions on multiple uniformly refined grids may have aided the discovery of the issues that caused the large variation in loudness measures of signatures provided by different particpants on the same grid.

A grid size recommendation for adapted grids should be established. Many adapted grid submissions utilized very small grids. This contributed to faster execution time, which is desirable in a production analysis or design setting. However, under resolved signatures due to a small number of control volumes could be misinterpreted as a failure of the adaptive grid scheme. Requesting both intermediate and final adapted signatures would indicate how the PL and $V$ measures converged as compared to uniform grid refinement and would verify the correctness of the adaptive method. ${ }^{12,48,49}$

The LM1021 signature and loudness metrics are sensitive to lift. ${ }^{43}$ The computed lift should have been 
gathered from participants and reported as a tool to understand the differences in near-body signatures and propagated ground signature loudness. An alternative would have been to run the CFD simulations at a specified lift condition and request angle of attack, which was done in the DPW series. The LM1021 extraction macro has about a quarter of resolution in terms of points per body length than the SEEBALR or DWB. The sensitivity of propagated signature to the resolution of the extraction macro should be understood.

The far-field correction ${ }^{30}$ was significant for the LM1021 at the extraction distances where high quality wind tunnel data was available. This indicates that directly propagating the participant submissions with an augmented Burgers code from these locations is not as appropriate as it is with the SEEB-ALR and DWB. The wind tunnel mounting hardware and the thicker boundary layer at the wind tunnel Reynolds number negatively impacted the signature of the model that is designed to be a quiet full-scale free-flight model. The differences between wind tunnel test and fight would be a relevant topic for future study and inclusion in a subsequent workshop.

\section{Next Steps}

The NASA Supersonics Project sonic boom goal for future supersonic aircraft is 65 to 70 PL (dB). ${ }^{50}$ Henne $^{15}$ proposes a small quiet supersonic jet (QSJ) with 68 to $85 \mathrm{PL}$ (dB). Kubota ${ }^{51}$ suggests a target of 88 PL (dB) in order to attain an acceptability of $90 \%$ in laboratory experiments. The configurations for SBPW1 were significantly louder: the DWB was approximately 96 PL (dB), the SEEB-ALR was approximately 92 PL (dB), and the LM1021 was 84-94 PL (dB). Examining submissions from quieter configurations with the methods developed in conjunction with SBPW-1 would maintain the relevancy of future sonic boom workshops.

Having a range of geometric complexity was important to the participants of SBPW-1. A future workshop should include both an axisymmetric configuration and a full-configuration (wing, body, tail, and nacelle) with complex near-field signatures that produce mid-80s to mid-70s PL on the ground. Having axisymmetric configuration that produces a signature with the complexity of the LM1021 may help to isolate geometry complexity as a source of PL variation. A formation of multiple axisymmetric bodies may isolate the spanwise distribution of acoustic disturbances as a source of PL variation.

Unfortunately, a cursory survey of publicly available geometries with wind tunnel measurements did not yield a sufficiently quiet configuration. This leaves the possibility of performing the next workshop on a new configuration. There is a risk that this new configuration may never be tested in a wind tunnel, but DPW-4 ${ }^{52}$ accepted this risk by holding workshop with an untested model and then successfully advocated for a test of that model after the workshop. Model supports and thicker boundary layers at lower Reynolds number required for supersonic wind tunnel testing can impact a design that is optimized for full-scale free-flight. It may be desirable to study a vehicle in both full-scale free-flight and wind tunnel test configurations.

The propagation of the near-field signatures to the ground and the loudness calculation from ground signatures can introduce additional uncertainty sources to sonic boom prediction. West et al. ${ }^{43}$ described a method of propagating uncertainties in the near-field CFD and atmospheric boom propagation, which showed that uncertainty in the atmospheric conditions can contribute more to PL uncertainty than the nearfield CFD alone. Therefore, future workshops should include an examination of propagation and loudness calculation methods to gain additional insight on the entire uncertainty budget of sonic boom prediction.

The impact of undershoots and overshoots, commonly present in near-field CFD signatures, has not been completely quantified for sonic boom prediction. Propagation methods, particularly methods that include molecular relaxation, filter the high frequency content present in near-field signature undershoots and overshoots. Direct frequency analysis of the near-field signature could be used to quantify impact of high frequencies the how they are filtered by the propagation method. ${ }^{53}$ Digital filtering of near-field and propagated signatures could be used understand what sections of the signature contribute most to the variation in high frequencies that could be impacting PL. ${ }^{44}$

This summary focused on the PL noise measure due to its published correlation to annoyance ${ }^{17}$ and its specification as a design target. ${ }^{15,50,51}$ Recent experiments have indicated that other measures are correlated with annoyance, particularly for sonic booms experienced indoors. ${ }^{54-56}$ Future workshops should consider these measures in addition to PL to understand if they place different requirements on near-field CFD or propagation methods because these measures emphasize different portions of the frequency spectrum. 


\section{Conclusions}

A summary and statistical analysis of the First AIAA Sonic Boom Prediction Workshop (SBPW-1) has been presented. Submissions from 19 groups are presented for two required and one optional configuration. These configurations captured a range of geometric complexity. They are an axisymmetric body with flattop ground signature (SEEB-ALR), a simple non-lifting wing body with a N-wave signature (DWB), and a complex configuration with wing, tail, flow-through nacelles, and blade mount sting (LM1021).

The participants provided near-field pressure signatures extracted from CFD computations at specific locations. The SEEB-ALR had 64 submission, the DWB had 60 submissions, and the optional LM1021 had 11 submissions. Many participants generated custom grids and solution adapted grids based on their best practices. The majority of participant groups provided multiple submissions computed with a series of uniformly refined committee provided grids. Submissions produced on these uniformly refined grids enabled a unique opportunity to study the grid convergence of a wide range of CFD methods for metrics of interest to the sonic boom research community.

The provided near-field signatures were compared to each other and wind tunnel measurements. A validation metric that quantifies the difference between the provided signatures and wind tunnel measurements was computed. The near-field CFD and wind tunnel signatures were propagated to the ground for comparison and the loudness measure PL was computed.

Statistics of validation metrics and loudness measures were computed for the two required SEEB-ALR and DWB configurations. The SEEB-ALR had a quieter median 91.8 PL (dB) than the DWB on the centerline, 95.5 PL (dB). The DWB median increased with off-track angle. All the median values for the required cases were within $0.5 \mathrm{~dB}$ of propagated wind tunnel measurements and often within $0.1 \mathrm{~dB}$. The inclusion or exclusion of the coarse grids of the uniform refinement study had a negligible impact on median and limit statistics. The limits were about $\pm 0.8 \mathrm{~dB}$ for the SEEB-ALR and $\pm 0.2 \mathrm{~dB}$ for the DWB. The uniform grid refinement study on the committee provided grids showed better convergence for the SEEB-ALR than the DWB for PL and the validation metric. Scatter was reduced with grid refinement for the configurations and metrics examined, except for the DWB validation metric. The DWB model produces strong shocks, which may have impeded the convergence of the validation metric.

The optional LM1021 had eleven submissions with larger scatter in PL than the two required cases. The differences between Euler, laminar, and turbulent submissions were noted in the near-field and propagated ground signatures. The PL measure had a large variation between methods and at different extraction location for the same method. The CSEL measure had less variation with extraction location. The louder PL submissions on the centerline were also loudest off track. A pair of signatures was identified that appeared very similar at the near field, but had almost a $3 \mathrm{~dB}$ PL difference when propagated to the ground with sBOOM.

The decay of the far-field correction with extraction distance was documented in terms of impulse and its impact on PL for this configuration. Impulse smoothly decreased with distance, but the PL correction had different behavior at each off track angle. The PL correction has more than one dB at some off-track angles at 25 half spans. Equivalent area computed from near-field signatures was presented as a function of extraction distance. The Euler submissions had a lower equivalent area than viscous submissions. Most submissions exhibited a drop in area between the furthest two extraction locations indicating that grid resolution was insufficient to maintain the signature.

Recommendations for future sonic boom workshops were provided with a discussion of the next steps in preparation for a potential follow-on workshop. These recommendations identified weaknesses in the analysis methods and opportunities to facilitate better understanding at future workshops.

\section{Acknowledgments}

The authors would like to thank the entire SBPW-1 organizing committee, participants, and attendees. The discussions during the workshop and in the hallways helped to craft the content of this summary. Sriram Rallabhandi provided the atmospheric propagation tool sBOOM and assistance on its use. Alexandra Loubeau provided the loudness measure calculation tool, guidance on its use, and detailed background on noise measures. Tom West, Bryan Reuter, Bil Kleb, and Eric Walker participated in invaluable discussions and developed a sonic boom uncertainty quantification method ${ }^{43}$ that shares many elements with the analysis in this summary. Joe Morrison and Chris Rumsey shared their statistical experience on the DPW and High 
Lift Prediction Workshop series. Mike Aftosmis suggested an explanation of the convergence of the DWB validation metrics. Cetin Kiris supplied observations of differences in the SEEB-ALR signatures. Juliet Page provided invaluable suggestions on evaluating the submissions and provided feedback on a draft of this summary. The work of the first author was supported under the High Speed Project of NASA's Fundamental Aerodynamics Program.

\section{References}

${ }^{1}$ Hemsch, M. J., "Statistical Analysis of Computational Fluid Dynamics Solutions from the Drag Prediction Workshop," AIAA Journal of Aircraft, Vol. 41, No. 1, 2004, pp. 95-103. 2011.

${ }^{2}$ Aftosmis, M. J., Nemec, M., and Cliff, S. E., "Adjoint-Based Low-Boom Design with Cart3D," AIAA Paper 2011-3500,

${ }^{3}$ Waithe, K. A., "Introduction of First Low Boom Prediction Workshop," AIAA Paper 2013-650, 2013.

${ }^{4}$ Darden, C. M., "Sonic-Boom Minimization with Nose-Bluntness Relaxation," NASA TP-1348, Jan. 1979.

${ }^{5}$ Makino, Y. and Noguchi, M., "Near-Field Pressure Measurements of Several Models in JAXA's 1m x 1m Supersonic Wind Tunnel," AIAA Paper 2013-648, 2013.

${ }^{6}$ Morgenstern, J. M., Buonanno, M., and Marconi, F., "Full Configuration Low Boom Model and Grids for 2014 Sonic Boom Prediction Workshop," AIAA Paper 2013-647, 2013.

${ }^{7}$ Park, M. A., Aftosmis, M. J., Campbell, R. L., Carter, M. B., Cliff, S. E., and Bangert, L. S., "Summary of the 2008 NASA Fundamental Aeronautics Program Sonic Boom Prediction Workshop," AIAA Paper 2013-649, 2013.

${ }^{8}$ Levy, D. W., Laflin, K. R., Tinoco, E. N., Vassberg, J. C., Mani, M., Rider, B., Rumsey, C. L., Wahls, R. A., Morrison, J. H., Brodersen, O. P., Crippa, S., Mavriplis, D. J., and Murayama, M., "Summary of Data from the Fifth AIAA CFD Drag Prediction Workshop," AIAA Paper 2013-46, 2013.

${ }^{9}$ Morrison, J. H., "Statistical Analysis of CFD Solutions from the Fifth AIAA Drag Prediction Workshop," AIAA Paper 2013-47, 2013.

${ }^{10}$ Rumsey, C. L. and Slotnick, J. P., "Overview and Summary of the Second AIAA High Lift Prediction Workshop," AIAA Paper 2014-747, 2014.

${ }^{11}$ DeBonis, J. R., Oberkampf, W. L., Wolf, R. T., Orkwis, P. D., Turner, M. G., Babinsky, H., and Benek, J. A., "Assessment of Computational Fluid Dynamics and Experimental Data for Shock Boundary-Layer Interactions," AIAA Journal, Vol. 50, No. 4, April 2012, pp. 891-903.

${ }^{12}$ Aftosmis, M. J. and Nemec, M., "Cart3D Simulations for the First AIAA Sonic Boom Prediction Workshop," AIAA Paper 2014-558, 2014.

13 "Civil Aircraft Sonic Boom," Code of Federal Regulations, Title 14, Pt. 91.817, 2011.

${ }^{14}$ Liebhardt, B., Linke, F., and Dahlmann, K., "Supersonic Deviations: Assessment of Sonic-Boom-Restricted Flight Routing," AIAA Journal of Aircraft, 2014.

${ }^{15}$ Henne, P. A., "Case for Small Supersonic Civil Aircraft," AIAA Journal of Aircraft, Vol. 42, No. 3, May-June 2005, pp. $765-774$.

${ }^{16}$ Sakata, K., "Japan's Supersonic Technology and Business Jet Perspectives," AIAA Paper 2013-21, 2013.

${ }^{17}$ Leatherwood, J. D., Sullivan, B. M., Shepherd, K. P., McCurdy, D. A., and Brown, S. A., "Summary of Recent NASA Studies of Human Response to Sonic Booms," The Journal of the Acoustical Society of America, Vol. 111, No. 1, Jan. 2002, pp. 586-598.

${ }^{18}$ Stevens, S. S., "Perceived Level of Noise by Mark VII and Decibels (E)," Journal of the Acoustical Society of America, Vol. 51, No. 2B, 1972, pp. 575-601.

${ }^{19}$ Shepherd, K. P. and Sullivan, B. M., "A Loudness Calculation Procedure Applied to Shaped Sonic Booms," NASA TP-3134, Nov. 1991.

${ }^{20}$ U.S. Standard Atmosphere, 1976, U.S. Government Printing Office, 1976.

${ }^{21}$ Morgenstern, J., Norstrud, N., Sokhey, J., Martens, S., and Alonso, J. J., "Advanced Concept Studies for Supersonic Commercial Transports Entering Service in the 2018 to 2020 Period," NASA CR-2013-217820, NASA Langley Research Center, Feb. 2013.

${ }^{22}$ Rallabhandi, S. K., "Advanced Sonic Boom Prediction Using the Augmented Burgers Equation," AIAA Journal of Aircraft, Vol. 48, No. 4, July-Aug. 2011, pp. 1245-1253.

${ }^{23}$ Rallabhandi, S. K., "Sonic Boom Adjoint Methodology and its Applications," AIAA Paper 2011-3497, 2011.

${ }^{24}$ American National Standards Institute, "Method for Calculation of the Absorption of Sound by the Atmosphere," ANSI S1.26-1995, Sept. 1995.

${ }^{25}$ Cleveland, R. O., Propagation of Sonic Booms Through a Real, Stratified Atmosphere, Ph.D. thesis, University of Texas at Austin, May 1995.

${ }^{26}$ Oberkampf, W. L. and Roy, C. J., Verification and Validation in Scientific Computing, Cambridge University Press, 2010.

${ }^{27}$ Park, M. A., Campbell, R. L., Elmiligui, A., Cliff, S. E., and Nayani, S. N., "Specialized CFD Grid Generation Methods for Near-Field Sonic Boom Prediction," AIAA Paper 2014-115, 2014.

${ }^{28}$ Cliff, S. E., Durston, D. A., Elmiligui, A., Jensen, J. C., and Chan, W. M., "Computation and Experimental Assessment of Models for the First AIAA Sonic Boom Prediction Workshop," AIAA Paper 2014-560, 2014.

${ }^{29}$ Hunton, L. W., Hicks, R. M., and Mendoza, J. P., "Some Effects of Wing Planform on Sonic Boom," NASA TN D-7160, NASA Ames Research Center, Jan. 1973.

42 of 43 
${ }^{30}$ Page, J. A. and Plotkin, K. J., "An Efficient Method for Incorporating Computational Fluid Dynamics into Sonic Boom Prediction," AIAA Paper 91-3275, 1991.

${ }^{31}$ Carlson, H. W. and Morris, O. A., "Wind-Tunnel Sonic-Boom Testing Techniques," AIAA Journal of Aircraft, Vol. 4, No. 3, 1967, pp. 245-249.

${ }^{32}$ Wilcox, Jr., F. J. and Elmiligui, A. A., "Experimental Measurements of Sonic Boom Signatures Using a Continuous Data Acquistion Technique," NASA TP-2013-218035, Aug. 2013.

${ }^{33}$ Castner, R., Shaw, S., Adamson, E., and Simerly, S., "Background Pressure Profiles for Sonic Boom Vehicle Testing in the NASA Glenn 8-foot by 6-foot Supersonic Wind Tunnel," AIAA Paper 2013-3122, 2013.

${ }^{34}$ Morgenstern, J. M., "Distortion Correction for Low Sonic Boom Measurement in Wind Tunnels," AIAA Paper 2012-3216, 2012.

${ }^{35}$ Nagai, S. and Iijima, H., "Uncertainty Identification of Supersonic Wind-Tunnel Testing," AIAA Journal of Aircraft, Vol. 48, No. 2, March-April 2011, pp. 567-577.

${ }^{36}$ Morgenstern, J. M., "How to Accurately Measure Low Sonic Boom or Model Surface Pressures in Supersonic Wind Tunnels," AIAA Paper 2012-3215, 2012.

${ }^{37}$ Cliff, S. E., Elmiligui, A. A., Aftosmis, M. J., Thomas, S. D., Morgenstern, J. M., and Durston, D. A., "Design and Evaluation of a Pressure Rail for Sonic Boom Measurement in Wind Tunnels," Seventh International Conference on Computational Fluid Dynamics (ICCFD7), July 2012.

${ }^{38}$ Durston, D. A., Cliff, S. E., Wayman, T. R., Merret, J. M., Elmiligui, A. A., and Bangert, L. S., "Near-Field Sonic Boom Test on Two Low-Boom Configurations Using Multiple Measurement Techniques at NASA Ames," AIAA Paper 2011-3333, 2011.

${ }^{39}$ Jackson, Jr., C. M., Corlett, W. A., and Monta, W. J., "Description and Calibration of the Langley Unitary Plan Wind Tunnel," NASA TP-1905, Nov. 1981.

${ }^{40}$ Bretl, K. N. and Walker, E. L., "Impact of Wind Tunnel Sonic Boom Measurement Uncertainty on Perceived Ground Noise," LARSS Final Report, NASA Langley Research Center, Aug. 2012.

${ }^{41}$ Nayani, S. N. and Campbell, R. L., "Evaluation of Grid Modification Methods for On- and Off-Track Sonic Boom Analysis," AIAA Paper 2013-798, 2013.

${ }^{42}$ Carter, M. B., Campbell, R. L., and Nayani, S. N., "USM3D Analysis of Low Boom Configuration," AIAA Paper 2011-3335, 2011.

${ }^{43}$ West, IV, T. K., Reuter, B. W., Walker, E. L., Kleb, B., and Park, M. A., "Uncertainty Quantification and Certification Prediction of Low-Boom Supersonic Aircraft Configurations," AIAA Aviation and Aeronautics Forum and Exposition (AVIATION 2014), American Institute of Aeronautics and Astronautics, Reston, VA (submitted for publication).

${ }^{44}$ Rallabhandi, S. K., private communication, 2014.

${ }^{45}$ Whitham, G. B., "The Flow Pattern of a Supersonic Projectile," Communications on Pure and Applied Mathematics, Vol. 5, No. 3, Aug. 1952, pp. 301-348.

${ }^{46}$ Walkden, F., "The Shock Pattern of a Wing-Body Combination, Far From the Flight Path," Aeronautical Quarterly, Vol. IX, No. 2, May 1958, pp. 164-194.

${ }^{47}$ Venkatakrishnan, V., "Convergence to Steady State Solutions of the Euler Equations on Unstructured Grids with Limiters," Journal of Computational Physics, Vol. 118, No. 1, 1995, pp. 120-130.

${ }^{48}$ Park, M. A. and Darmofal, D. L., "Validation of an Output-Adaptive, Tetrahedral Cut-Cell Method for Sonic Boom Prediction," AIAA Journal, Vol. 48, No. 9, Sept. 2010, pp. 1928-1945.

${ }^{49}$ Alauzet, F. and Loseille, A., "High-Order Sonic Boom Modeling Based on Adaptive Methods," Journal of Computational Physics, Vol. 229, No. 3, 2010, pp. 561-593.

50 "Research Opportunities in Aeronautics - 2008 (ROA-2008)," Amendment 7 to the NASA Research Annoucnements (NRA), Appendix A-4 NNH08ZEA001N, March 2008.

${ }^{51}$ Kubota, H., "Sonic Boom Research in Japan," AIAA Paper 2003-3578, 2003.

${ }^{52}$ Morrison, J. H., "Statistical Analysis of CFD Solutions from the Fourth AIAA Drag Prediction Workshop," AIAA Paper 2010-4673, 2010.

${ }^{53}$ Page, J. A., private communication, 2014.

${ }^{54}$ Loubeau, A., Sullivan, B. M., Klos, J., Rathsam, J., and Gavin, J. R., "Laboratory Headphone Studies of Human Response to Low-Amplitude Sonic Booms and Rattle Heard Indoors," NASA TM-2013-217975, Feb. 2013.

${ }^{55}$ Rathsam, J., Loubeau, A., and Klos, J., "Simulator Study of Indoor Annoyance Caused by Shaped Sonic Boom Stimuli with and without Rattle Augmentation," 27th National Conference on Noise Control Engineering 2013 (Noise-Con 13), Aug. 2013.

${ }^{56}$ Page, J. A., Hodgdon, K. K., Krecker, P., Cowart, R., Hobbs, C., Wilmer, C., Koening, C., Holmes, T., Gaugler, T., Shumway, D. L., Rosenberger, J. L., and Philips, D., "Waveforms and Sonic Boom Perception and Response (WSPR): Low-Boom Community Response Program Pilot Test Design, Execution, and Analysis," NASA CR-218180, March 2014. 